\title{
Graph Calculus and the Disconnected-Boundary Schwinger-Dyson Equations of Quartic Tensor Field Theories
}

\section{Carlos I. Pérez-Sánchez ${ }^{1,2}$}

Received: 13 January 2020 / Accepted: 24 June 2020 / Published online: 9 November 2020 (C) The Author(s) 2020

\begin{abstract}
Tensor field theory (TFT) focuses on quantum field theory aspects of random tensor models, a quantum-gravity-motivated generalisation of random matrix models. The TFT correlation functions have been shown to be classified by graphs that describe the geometry of the boundary states, the so-called boundary graphs. These graphs can be disconnected, although the correlation functions are themselves connected. In a recent work, the Schwinger-Dyson equations for an arbitrary albeit connected boundary were obtained. Here, we introduce the multivariable graph calculus in order to derive the missing equations for all correlation functions with disconnected boundary, thus completing the Schwinger-Dyson pyramid for quartic melonic ('pillow'-vertices) models in arbitrary rank. We first study finite group actions that are parametrised by graphs and build the graph calculus on a suitable quotient of the monoid algebra $\mathcal{A}[G]$ corresponding to a certain function space $\mathcal{A}$ and to the free monoid $G$ in finitely many graph variables; a derivative of an element of $\mathcal{A}[G]$ with respect to a graph yields its corresponding group action on $\mathcal{A}$. The present result and the graph calculus have three potential applications: the non-perturbative large- $N$ limit of tensor field theories, the solvability of the theory by using methods that generalise the topological recursion to the TFT setting and the study of 'higher dimensional maps' via Tutte-like equations. In fact, we also offer a term-by-term comparison between Tutte equations and the present Schwinger-Dyson equations.
\end{abstract}

Keywords Tensor models · Quantum field theory · Schwinger-Dyson Equations · Matrix models $\cdot$ Random maps $\cdot$ Tutte equations

Mathematics Subject Classification (2010) 81Txx (primary) - Secondary 20Nxx · 05Exx

Carlos I. Pérez-Sánchez

cperez@fuw.edu.pl

Extended author information available on the last page of the article. 


\section{Introduction and Motivation}

The quest for laws of physics near the Planck scale leads some quantum gravitologist and quantum cosmologists to replace the smooth space-time paradigm with new geometrical structures that are suitable for said energy scale. Those new structures include discretisation of space-time (e.g. causal dynamical triangulations [2]), the algebraisation of space-time (e.g. noncommutative geometry [16, 42]), just to name some. ${ }^{1}$ Already the sole description of a space-time by a single mathematical object is expected to require therefore novel geometrical ideas.

If one adopts a path-integral approach, the exploration of the quantum theory of space-time requires additionally a multi-geometry description, to which 'offshell' geometries also contribute. Each of these geometries $\xi$ is weighted via $\exp (\mathrm{i} S(\xi) / \hbar) \mathrm{d} \xi$ by a 'classical' action $S(\xi)$, bounded to resemble the EinsteinHilbert action in the classical limit, in which $\xi$ starts looking like a Riemannian or Lorentzian manifold.

Random tensors $[1,10,28,56]$ and related theories construct these kinds of measures $\mathrm{d} \xi$, offer precisely a built-in description of both random and discrete geometry in arbitrary dimensions, and therefore constitute a tool to test models of background independent quantum gravity (see e.g. [23]). Interest in the study of Euclidean quantum field theory (QFT) aspects of random tensors leads to tensor field theory (TFT) $[9,11,49,58,61]$, the matter of this article. TFT fits in a 'QFT $+\epsilon$ ' framework, that is to say a conservative modification of QFT, which one can pursue in the perturbative or non-perturbative approaches. The origin of TFT can be traced back to Group Field Theories [17, 27, 29, 36, 46] (in fact, for abelian GFT's the present results hold, even though not explicitly stated).

Non-perturbative TFT deals with the geometry of boundary states. Single geometries in TFT are represented by certain decorated graphs called coloured graphs; this decoration is precisely the information that allows the construction of PL-manifolds from graphs. Bulk-geometry graphs - Feynman diagrams of TFT - have a colour more than the graphs that triangulate the boundary geometries, which are called therefore boundary graphs ( $\partial$-graphs, for short). The two-fold purpose of this article is to define in abstract way a calculus with coloured boundary-graph variables, and shortly afterwards, to apply this construct to a particular problem in non-perturbative TFT.

The single-variable graph calculus has been used there as a toolkit for nonperturbative field tensor theory, leading to the full Ward-Takahashi identity [53]. The single-variable graph calculus allows to define each correlation function as a graph derivative of the free energy. Boundary graphs turn out to classify the correlation

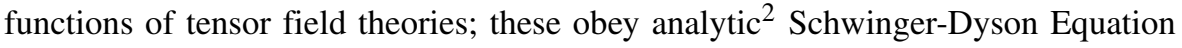
(SDE). SDE for tensor models, which do reduce to the matrix models loop equations

\footnotetext{
${ }^{1}$ See for instance [43] for a thorough classification.

${ }^{2}$ We write 'analytic' as opposed to algebraic SDE for expectation values. We conceive tensor field theory as a discretisation (therefore, 0 -dimensional) of a $D$-dimensional quantum field theory. The $2 k$-point correlation functions are thus functions of $\mathbb{Z}^{k \times D} \rightarrow \mathbb{C}$ which in a continuous-index limit pass to functions $\mathbb{R}^{k \times D} \rightarrow \mathbb{C}$, and render the SDE integro-differential equations.
} 
for rank-2 models, were found by Gurău [30, 31]. Gurău's work [29] is crucial for the modern and evolved TFT. However, the method presented here does not use Gurău's results on SDE; in fact, the objects described here differ from those considered in tensor models: while tensor model SDE describe relations between expectation values of observables, the SDE of tensor field theory are obeyed by correlation functions (Green's functions). The interest on the latter relies on Constructive Tensor Field Theory [57] and might find application in Group Field Theory. Unless otherwise stated, the SDE we refer to are those of TFT.

Each and every analytic SDE for a connected correlation function corresponding to a connected, but otherwise arbitrary, boundary graph was presented in [51] in terms of a general formula that relates a given correlation function with its neighbourings (relative to the number of points) in terms of simple graph operations. In order to obtain these results, the single-variable graph calculus was a useful tool, which however does not assist any longer in the the derivation of SDE for connected correlation functions with disconnected boundary. This derivation requires a multivariable graph calculus, the variables being the different boundary components. The graph calculus introduced here is general and abstract -in particular it does not depend on the details of the theory (dynamics, symmetries) — but it is meant as a tool for TFTs with complex tensors. It therefore specializes in bipartite graphs.

We introduce graph-group actions as the basis of the multivariable calculus, and study their generating functionals. Concretely, we obtain formulae for the graph derivative of products of functionals, i.e. the corresponding Leibniz rule that generalises

$$
\partial^{\boldsymbol{\alpha}}(F \cdot G)=\sum_{0 \leq \boldsymbol{\gamma} \leq \boldsymbol{\alpha}}\left(\begin{array}{l}
\boldsymbol{\alpha} \\
\boldsymbol{\gamma}
\end{array}\right) \partial^{\gamma} F \cdot \partial^{\boldsymbol{\alpha}-\boldsymbol{\gamma}} G \quad \text { (in multi-index notation), }
$$

when one replaces usual partial derivatives $\partial_{\mu}$ (say, with $x^{\mu}$ a coordinate of $\mathbb{R}^{n}$, and $F, G$ real-valued smooth functions there) by derivatives $\partial_{g}=\partial / \partial g$ with respect to a graph $g$. The formula for graphs takes a different form, but reduces, as it should, to (1.1) when one replaces functionals with functions and simultaneously considers trivial group actions.

We prove that this abstract structure underlies tensor models functionals and use it to find a general formula for the SDE of the quartic 'pillow'-model, for the connected correlation functions with arbitrary disconnected boundary (abbr. disconnected- $\partial$ ). This is the missing piece that complements the connected-boundary SDE-pyramid obtained in [51]. To have it complete is important for the analysis of the nonperturbative large- $N$ limit of tensor field theories. Moreover, although it is not clear which recursion should generalise the topological recursion [25], it is clear that the disconnected- $\partial$ correlation functions play an important role. ${ }^{3}$

The geometric spirit of taking graph-derivatives can be understood as the tensor field theory counterpart of 'taking residues' in matrix models (cf. [26, Ch. 1-2])

$$
\mathcal{T}_{l_{1} \ldots l_{\kappa}}=\operatorname{Res}_{x_{1} \rightarrow \infty} \ldots \operatorname{Res}_{x_{\kappa} \rightarrow \infty}\left[x_{1}^{l_{1}} \cdots x_{\kappa}^{l_{\kappa}} W_{\kappa}\left(x_{1}, \ldots, x_{\kappa}\right)\right]
$$

\footnotetext{
${ }^{3}$ For instance, higher dimensional analogue of the 'pair of pants' being represented by a correlation function with three melonic boundary components.
} 
in order to project the free energy $W_{\kappa}$ (with $\kappa$ boundaries) of a matrix model onto the generating function $\mathcal{T}_{l_{1} \ldots l_{\kappa}}$ of random maps with $\kappa$ marked faces with fixed perimeter lengths $\left(l_{1}, \ldots, l_{\kappa}\right)$. After the well-known equivalence [26, Thm. 2.5.1] between Tutte equations [63, 64] for the enumeration of random maps and the loop equations for a suitable matrix model [44] (also summarised here in in Section 5.2), we can state the present result as the basis to obtain Tutte-like equations for the higher-dimensional analogue of the generating function of random maps $\mathcal{T}_{l_{1} \ldots l_{\kappa}}$. This improvement over [51] — where only the equations analogous to a single-boundary correlator, say $\mathcal{T}_{l_{1}}$ 's cousin, are presented — requires new developments: A straightforward generalisation of the proof given in [51] is impossible due to the absence of the multi-variable graph calculus. Although it would be possible to state the main result solely in a TFT context, the full notation would be a burden in the proof. We state some results in lighter notation and offer a shorter proof, at expenses of introducing some new concepts. The output of the main theorem is also a set of new graph operations that extend those used to describe the connected- $\partial$ SDE in [51]. Also the edge $\operatorname{swap}^{4} \varsigma_{a}$ (see Fig. 1), as unary operation on a connected graph, is extended to a binary operation implying two connected components; $\varsigma_{a}$ is then interpreted, following [52], as their connected sum.

This article is divided in an abstract part (Section 2, whose main results are Lemmas 2 and 3), and a TFT-part. The next section explains in detail the following mnemonics: for coloured graphs $g$ and $h$,

$$
\frac{\partial g}{\partial h}=\delta(h, g) \cdot \text { group action of } G(g) .
$$

Here $\delta(g, h)=1$ if the graphs $g$ and $h$ are isomorphic, and $\delta(g, h)$ vanishes otherwise; here $G(g)$ is a group determined by $g$. In Section 3 we make the connection between the two first sections and model-independent TFT. In Section 4, our quartic model is detailed and the results of the previous sections are applied to the main problem, namely to find the SDE for connected correlation functions with arbitrary disconnected boundary with arbitrary number of connected components. We highlight in Section 5, preparing an important future task to apply our results, the term-to-term parallel between Tutte equations and those presented here, as well as analogies in the derivation of both sets of equations. Concretely, we compare the new operations on the boundary graphs of TFT (Table 4) with their matrix models counterpart (Table 2). The operations and terms presented in the SDE of [51] are only the counterpart of those matrix models SDE presented in Table 1. Section 6 gives explicitly some of the SDE for 4, 6-point functions for rank-3 theories. The conclusions and outlook are given in Section 7 , discussing a potential application related to the higher-dimensional analogue of the topological recursion. The useful coefficients that encode the insertion of the 4-point functions into the 2-point function and the 6-point functions into the 4-point functions are given in the Appendix A.

\footnotetext{
4 This operation has been studied in the literature of Graph-Encoded Manifolds [39] and by the Crystallisation Theory [21].
} 

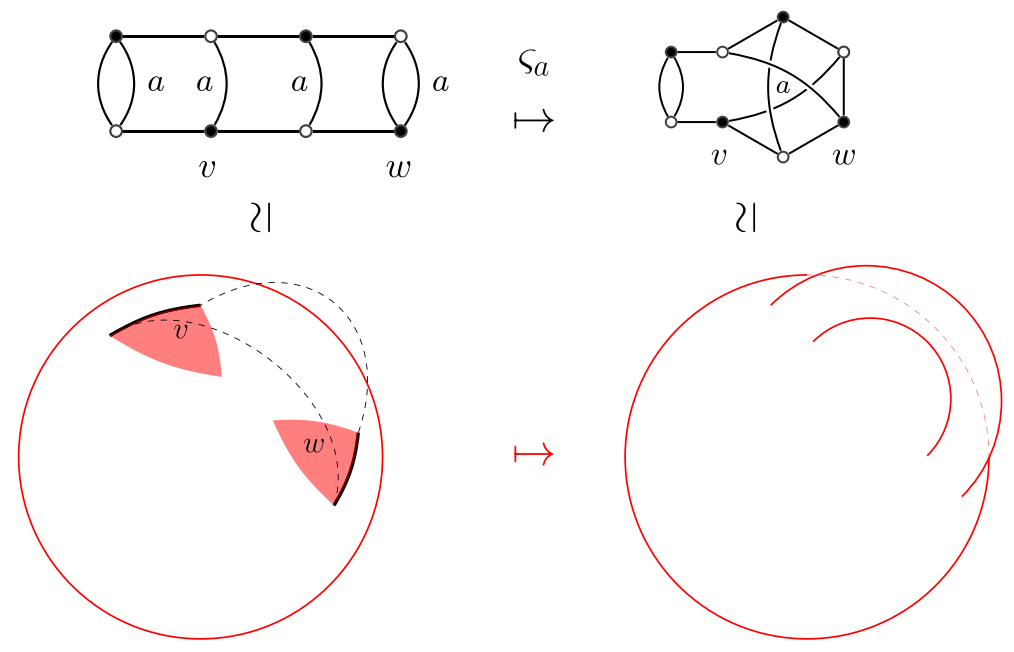

Fig. 1 An example of $a$-coloured edge swap increasing the genus of a connected graph

\section{Graph Calculus}

In this section we explain what we mean by graph calculus. For naturality reasons we consider the empty graph $\varnothing$ as coloured and add it to the set of (possibly) disconnected, closed, regularly edge- $D$-coloured, vertex-bipartite graphs (' $D$-coloured graphs') $\operatorname{Grph}_{D}^{\amalg, \mathrm{cl}}$, to form $\mathrm{G}_{D}=\{\varnothing\} \cup \mathrm{Grph}_{D}^{\amalg, \mathrm{cl}}$. This is in line with other theories (as a matter of fact Topological Quantum Field Theories) that need the empty $D$ manifold. Also, it is technically advantageous and not the first time it is considered, see for instance [35]. Last, but not least, it is physically ${ }^{5}$ required, since $\varnothing$ is related to the Fock vacuum in the second quantized formulation of TFT. Henceforth, all graphs are coloured, but other types of graphs could be used for the next constructs.

\subsection{Single Variable Graph Calculus}

We regard $\mathrm{G}_{D}$ with a monoidal structure, the product and the unit being given by

$$
c_{1} c_{2}=c_{1} \amalg c_{2} \quad \text { and } \quad c \amalg \varnothing=c=\varnothing \amalg c,
$$

respectively, for all $c, c_{1}, c_{2} \in \mathrm{G}_{D}$. We choose to remember the order of the factors, so this product is generally non-commutative, $c_{1} c_{2} \neq c_{2} c_{1}$.

Definition 1 (System of graph-group actions) For a finite collection $H \subset \mathrm{G}_{D}$, consider the following structures:

- $\quad$ for each connected graph $c \in H$ :

${ }^{5}$ An anonymous referee is acknowledged for this remark. 
- a set $\mathscr{N}(c)$ is associated with $c$; for the empty graph, $\mathscr{N}(\varnothing)=\{*\}$ is the singleton

- a finite group $G(c)$ and

- a group action $G(c) \curvearrowright \mathscr{N}(c)$ of $G(c)$ on $\mathscr{V}(c)$

- if $g=c_{1} c_{2} \cdots c_{n}$ is a factorisation in connected components $c_{i}$, then $\mathscr{V}(g)$ satisfies $^{6}$

$$
\mathscr{N}(g)=\mathscr{N}\left(c_{1}\right) \amalg \cdots \amalg \mathscr{N}\left(c_{n}\right)
$$

The collection $\{\mathscr{V}(g), G(g)\}_{g \in H}$ is a system of graph-group actions.

If additionally, for each graph in $g \in H$, one has functions

$$
u_{g}: \mathscr{N}(g) \rightarrow \mathbb{C}(\text { or } \mathbb{R}),
$$

then one says that $\left\{u_{g}, \mathscr{N}(g), G(g)\right\}_{g \in H}$, or more succinctly, $\left\{u_{g}\right\}_{g \in H}$ is a family of functions supported on $\{\mathscr{N}(g), G(g)\}_{g \in H}$.

We are interested in triples $\left\{u_{g}, \mathscr{N}(g), G(g)\right\}_{g \in H}$ and formal sums of the type $U=$ $\sum_{h \in H} u_{h} h$, which we refer to as their generating functionals. In this case we say that (the set of graphs) $H$ spans $U$. Why these are functionals instead of functions will become apparent while addressing the applications. At this point also the following terminology, inherited from the physical significance, might seem mysterious: we call the elements of $\mathscr{V}(g)$ the momenta of the graph $g$. Notice that $u_{\varnothing}$ is a constant.

As the last reference to TFT in this section, we clarify that the nature of these graphs is not important at this point; examples will be presented in later sections. We just clarify the reader that is well-versed with tensor models, that graphs treated here are not Feynman graphs, but boundary graphs of these in a rank- $D$ TFT. In this context, functions $u_{g}$ are unknown, ${ }^{7}$ and one derives equations that they should satisfy. Only after knowing solutions we would be able to fix a function space $u_{g}$ should belong to, which is for now unspecified. We vaguely refer then to them as 'functions'.

Next, some words on notation. For a factorisation $g=c_{1} \cdots c_{n}$ in connected graphs $c_{j} \neq \varnothing$, we let $g / c_{i}$ be the graph with the $i$-th connected component deleted,

$$
g / c_{i}:=c_{1} \ldots \widehat{c_{i}} \ldots c_{n}=c_{1} \ldots c_{i-1} c_{i+1} \ldots c_{n} .
$$

Notice that this deletion does not only care about the graph-class, but also about its spot in the factorisation, which we can keep track of thanks to the monoidal structure of $\mathrm{G}_{D}$.

For $g=c_{1} \cdots c_{n}$ as before, let $Y \in \mathscr{N}\left(c_{r}\right), 1 \leq r \leq n$. Given a function $v_{g}$ : $\mathscr{N}(g) \rightarrow \mathbb{C}$ we define the insertion of $Y$ in the $r$-th argument of $v_{g}$

$$
\iota_{Y}^{r} v_{g}: \mathscr{N}\left(c_{1}\right) \amalg \cdots \amalg \mathscr{N}\left(c_{r-1}\right) \amalg \mathscr{N}\left(c_{r+1}\right) \amalg \cdots \amalg \mathscr{V}\left(c_{n}\right) \rightarrow \mathbb{C},
$$

\footnotetext{
${ }^{6}$ One could relax this condition so that there exist domains $\mathscr{U}(g)$ of $\mathscr{V}(g)$ compatible with the $G(g)$-action. However, we keep the natural condition (2.1).

${ }^{7}$ For instance, $u_{g}$ can be the correlation functions.
} 
using (2.1) by

$$
\left(\iota_{Y}^{r} v_{g}\right)\left(X_{1}, \ldots, X_{r-1}, X_{r+1}, \ldots, X_{n}\right)=v_{g}\left(X_{1}, \ldots, X_{r-1}, Y, X_{r+1}, \ldots, X_{n}\right),
$$

where $X_{i} \in \mathscr{V}\left(c_{i}\right)$ for each $i \neq r$.

Definition 2 Let $H \subset \mathrm{G}_{D}$ span the functional $U=\sum_{g} u_{g} g$. Given any connected graph $h \in \mathrm{G}_{D}, h \neq \varnothing$, and an arbitrary graph $g=c_{1} \cdots c_{n}$ factorised in connected components $c_{i}$, we define $I(g, h)=\left\{i \in \llbracket 1, n \rrbracket \mid h=c_{i}\right\}$; that is, $I(g, h)$ is the subset of numbers that indexes the factors of $g$ that coincide with $h$. For $r \in I(g, h)$, we label by $h^{(r)}$ the appearance of $h$ in the $r$-th factor of $g=c_{1} \cdots c_{n}$. We define the functional graph derivative with respect to $h$ (evaluated at $X$ ) as the functional

$$
\frac{\delta U}{\delta h(X)}=\sum_{g \in H} \sum_{r \in I(g, h)} \sum_{\sigma \in G(h)} \iota_{\sigma(X)}^{r} u_{g}\left(g / h^{(r)}\right), \quad X \in \mathscr{U}(h) \subset \mathscr{V}(h) .
$$

The well-definedness follows from condition (2.1), which implies that in each case $\iota_{\sigma(X)}^{r} u_{g}$ is indeed a function on $\mathscr{N}\left(g / h^{(r)}\right)$. We stress that this derivative could be defined in a proper domain $\mathscr{U}(h)$ of $\mathscr{V}(h)$. Further, if $h$ occurs nowhere as a factor of $g$ the sum is empty, and thus $\delta U / \delta h \equiv 0$. The derivative with respect to $\varnothing$ is the coefficient of that graph, $\delta U / \delta \varnothing:=u_{\varnothing} \in \mathbb{C}$.

To clarify this definition, consider a monomial functional, $U=u_{g} g$, with $g=h^{n}$ for some integer $n \geq 1$ and $h$ a connected graph. By definition, one has

$$
\frac{\delta U}{\delta h(X)}=\sum_{r=1}^{n} \sum_{\sigma \in G(h)}\left(\iota_{\sigma(X)}^{r} u_{h^{n}}\right) h^{n-1}
$$

which one can rethink as

$$
\begin{aligned}
\frac{\delta\left(h^{n}\right)}{\delta h} & =\left(\frac{\delta h}{\delta h} h^{n-1}+h \frac{\delta h}{\delta h} h^{n-2}+\ldots+h^{n-1} \frac{\delta h}{\delta h}\right) \\
& =\left(G(h) h^{n-1}+h G(h) h^{n-2}+\ldots+h^{n-1} G(h)\right),
\end{aligned}
$$

if

$$
\left(\frac{\delta h}{\delta h} u_{h}\right)(X)=\sum_{\sigma \in G(h)}\left(\sigma \cdot u_{h}\right)(X)=\sum_{\sigma \in G(h)} u_{h}(\sigma X) .
$$

To illustrate the action in slightly more generality, if in $u_{c_{1} \ldots c_{r-1} h c_{r+1} \ldots}$ none of the $c_{j}$ is isomorphic to $h$, then

$$
\left(\frac{\delta h}{\delta h} u_{c_{1} \ldots c_{r-1} h c_{r+1} \ldots}\right)(X)=\sum_{\sigma \in G(h)} \iota_{\sigma(X)}^{r} u_{c_{1} \ldots c_{r-1} h c_{r+1} \ldots} .
$$

On this account, the useful symbolism to keep in mind is that the derivative of a graph with respect to itself is the group action of $G(h)$ on $\mathscr{N}(h)$,

$$
\frac{\delta h}{\delta h}=G(h) \curvearrowright\{\text { functions } \mathscr{N}(h) \rightarrow \mathbb{C}\} .
$$


In the sequel, we will often abuse on notation and write this equality without the curved action-arrow, as we already did above in (2.2). The moral is that each factor $h$ occurring in a term of the type

$$
u_{c_{1} \ldots c_{r-1} h c_{r+1} \ldots c_{n}}\left(c_{1} \ldots c_{r-1} h c_{r+1} \ldots c_{n}\right)
$$

is a potential $G(h)$-orbit of the $r$-th argument $u_{c_{1} \ldots c_{r-1} h c_{r+1} \ldots c_{n}}$.

For iterated derivatives with respect to $h$, one can see by induction that for $n \geq 2$, the iteration of $n$ graph derivatives applied to $h^{n}$ yields

$$
\frac{\delta^{n}\left(h^{n}\right)}{\delta h \delta h \cdots \delta h}\left(X_{1}, \ldots, X_{n}\right)=\sum_{\mu \in \mathfrak{S}(n)} \sum_{\left(\sigma^{1}, \ldots, \sigma^{n}\right) \in G(h)^{n}} \iota_{\sigma^{\mu(1)}\left(X_{1}\right)}^{1} \cdots \iota_{\sigma^{\mu(n)}\left(X_{n}\right)}^{n} .
$$

If $G_{i}(h)$ is the $i$-th factor of the group $G(h)^{n}$, a more transparent notation of last equation is

$$
\frac{\delta^{n}\left(h^{n}\right)}{\delta h \delta h \cdots \delta h}=\sum_{\mu \in \mathfrak{S}(n)} G_{\mu(1)}(h) G_{\mu(2)}(h) \cdots G_{\mu(n)}(h),
$$

where the group $G_{\mu(i)}(h)$ acts on the $i$-th factor of the set $\mathscr{V}(h) \amalg \cdots \amalg \mathscr{V}(h)$. The group corresponding to the $n$-th derivative of the $n$-th power of a graph $h$ with respect to itself is

$$
\frac{\delta^{n}\left(h^{n}\right)}{\delta h \delta h \cdots \delta h}=G(h)>\mathfrak{S}(n) .
$$

In this case, the wreath product $G(h)_{2} \mathfrak{S}(n)$ is the semi-direct product $G(h)^{n} \rtimes_{\psi} \mathfrak{S}(n)$, with the obvious action $\psi$ of $\mathfrak{S}(n)$ on the $n$ copies of $G(h)$.

To give further detail, given a generating system of graph-group actions $\{\mathscr{V}(g), G(g)\}_{g \in H}$ and $h \in H$, consider a function $F: \mathscr{V}(h) \amalg \cdots \amalg \mathscr{V}(h) \rightarrow \mathbb{C}$. An element $\Omega=(\sigma ; \mu)=\left(\sigma^{1}, \ldots, \sigma^{n} ; \mu\right)$ of the group in (2.7) acts as follows:

$$
(\Omega \cdot F)\left(X_{1}, \ldots, X_{n}\right)=F\left(\sigma^{1}\left(X_{\mu(1)}\right), \ldots, \sigma^{n}\left(X_{\mu(n)}\right)\right) .
$$

By departing from (2.9), the composition with another element $\Xi=(\tau, v)$ in the group (2.7) is easily proven to yield $\Xi \circ \Omega=\left(\boldsymbol{\tau} \psi_{\nu}(\sigma) ; \nu \mu\right)$, where

$$
\psi: \mathfrak{S}(n) \rightarrow \operatorname{Aut}\left(G(h)^{n}\right) \quad \mu \mapsto\left[\psi_{\mu}:\left(\sigma_{i}\right)_{i=1}^{n} \mapsto\left(\sigma_{\mu(i)}\right)_{i=1}^{n}\right],
$$

which is the product of $G(h)^{n} \rtimes_{\psi} \mathfrak{S}(n)$, as claimed.

\subsection{Examples of Graph-Group Action Systems}

Roughly stated, a multivariable graph calculus (of $n$ graph variables) consists of generating functionals of functions $u_{g}$ supported on a system of graph-group actions $\{\mathscr{V}(g), G(g)\}_{g \in H}$ that are spanned by a finite set $H$. We take $H \subset \operatorname{FM}\left(\left\{h_{1}, \ldots, h_{n}\right\}\right)$, the free monoid generated ${ }^{8}$ by $n$ non-isomorphic graphs $\mathfrak{h}=\left\{h_{1} \ldots, h_{n}\right\}$. For a

\footnotetext{
${ }^{8}$ We recall that the free monoid generated by $\mathfrak{h}=\left\{h_{1}, \ldots, h_{n}\right\}$ is in this case the following set $\mathrm{FM}(\mathfrak{h})=$ $\left\{l_{1} \cdots l_{m}: m \in \mathbb{Z}_{\geq 0}\right.$ and $\left.l_{i} \in \mathfrak{h}\right\}$ endowed with the concatenation operation; containing the empty graph, i.e. the empty word.
} 
multivariable graph calculus the key property is that the graph-group actions $G\left(h_{i}\right)$ are pairwise independent, that is for each $i, j=1, \ldots, n$,

$$
\frac{\delta h_{i}}{\delta h_{j}}=\delta_{j}^{i} G\left(h_{i}\right), \quad h_{i}, h_{j} \in \mathfrak{h} .
$$

For the special element $g=h_{1}^{\alpha_{1}} \cdots h_{n}^{\alpha_{n}}$ the restriction imposed by (2.10) implies

$$
\frac{\delta g}{\delta g}=G(g)=G\left(h_{1}\right)>\mathfrak{S}\left(\alpha_{1}\right) \times G\left(h_{2}\right) \imath \mathfrak{S}\left(\alpha_{2}\right) \times \cdots \times G\left(h_{n}\right)>\mathfrak{S}\left(\alpha_{n}\right) .
$$

Before formally defining multivariable graph calculus, the next examples are just meant to illustrate last action (2.11), rather than the role of the graphs in graphgenerated actions, and therefore can be skipped (to Section 2.3).

Example 1 Let $\zeta_{n} \neq 1$ denote a $n$-th root of unit $(n \geq 2)$, and consider the system of graph-group actions with a single graph $g$. Let $G(g)$ be the group spanned by $\zeta_{n}$ $\mathscr{N}(g)=\mathbb{C}$ by multiplication. Then the functional graph derivative of $g$ with respect to itself on the identity $\operatorname{id}_{\mathbb{C}}$ vanishes identically:

$$
\left(\frac{\delta g}{\delta g}\right) \operatorname{id}_{\mathbb{C}} \equiv 0 .
$$

The $G(g)$-orbit of the function $f_{n}: z \mapsto z^{n}$ yields

$$
\left(\frac{\delta g}{\delta g(z)}\right)\left(f_{n}\right)=n \cdot z^{n} \text {. }
$$

Example 2 Consider a finite set $H \subset \mathrm{FM}(\mathfrak{h})$ and the following graph-group actions system

$$
G(g)=\mathfrak{S}\left(\left|g^{0}\right|\right), \quad \mathscr{N}(g)=M_{\left|g^{0}\right| \times\left|g^{0}\right|}(\mathbb{R}) .
$$

Here $\left|g^{0}\right|$ is the number of vertices of $g$. The action of the symmetric group on the matrices permutes columns (or rows). Then the orbit of the determinant det: $\mathscr{N}(g) \rightarrow$ $\mathbb{R}$ vanishes identically. This follows from considering, for an arbitrary matrix $X=$ $\left(X_{a b}\right) \in \mathscr{N}(g)$,

$$
\begin{aligned}
\left(\frac{\delta g}{\delta g(X)}\right) \operatorname{det}(\bullet) & =\sum_{\sigma \in \mathfrak{S}\left(\left|g^{0}\right|\right)} \operatorname{det}\left(X_{\sigma(a) b}\right) \\
& =\sum_{\sigma \in A} \operatorname{det}\left(X_{\sigma(a) b}\right)+\sum_{\sigma \in \mathfrak{S}\left(\left|g^{0}\right|\right)-A} \operatorname{det}\left(X_{\sigma(a) b}\right) \\
& =\sum_{\sigma \in A} \operatorname{det}\left(X_{a b}\right)-\sum_{\sigma \in \mathfrak{S}\left(\left|g^{0}\right|\right)-A} \operatorname{det}\left(X_{a b}\right)=0,
\end{aligned}
$$

where $A$ is the alternating subgroup; its complement in the symmetric group consists of odd-degree permutations, whence the common minus sign in the last line. Both have the same order, which explains why the sum vanishes independently of $X$. 
Example 3 Let $D \in \mathbb{Z}_{\geq 1}$. Let two non-isomorphic graphs $H=\{g, h\} \subset \mathrm{G}_{D}$ parametrise the system of graph-group actions given by $\{\mathscr{V}(l), G(l)\}_{l \in H}$, being

$$
\begin{array}{lll}
\mu \in G(g)=\mathfrak{S}(D) & Z=\left(z_{i}\right)_{i} \in \mathscr{H}(g)=\mathbb{C}^{D} & \mu:\left(z_{i}\right) \mapsto\left(z_{\mu(i)}\right) \\
\tau \in G(h)=\mathbb{Z}_{2} & \varepsilon \in \mathscr{V}(h)=\mathbb{Z}_{2} & \varepsilon: s \mapsto \tau \varepsilon
\end{array}
$$

where $\mathbb{Z}_{2}$ is written multiplicatively $\{-1,1\}$. Let $F: \mathscr{V}(g) \amalg \mathscr{V}(g) \amalg \mathscr{V}(h) \rightarrow \mathbb{C}$ be given by, say,

$$
F(Z, W, \varepsilon)=\frac{\varepsilon}{2(D !)^{2}} \mathrm{e}^{-|W|+\varepsilon|Z|}=: c(D) \cdot \varepsilon \cdot \mathrm{e}^{-|W|+\varepsilon|Z|} .
$$

Then the functional graph derivative of $g^{2} f$ with respect to itself yields the following group-orbit, when applied to $F$ :

$$
\begin{aligned}
& \frac{\delta^{3}\left(g^{2} h\right)}{\delta g\left(Z_{1}\right) \delta g\left(Z_{2}\right) \delta h(\varepsilon)} F \\
= & \left(\frac{\delta^{3} g g h}{\delta g \delta g \delta h} F\right)\left(Z_{1}, Z_{2}, \varepsilon\right) \\
= & ([G(g) \imath \mathfrak{S}(2) \times G(h)] \cdot F)\left(Z_{1}, Z_{2}, \varepsilon\right) \\
= & \sum_{\mu \in \mathfrak{S}(2)} \sum_{\tau \in \mathbb{Z}_{2}} \sum_{\sigma \in \mathfrak{S}(D)} \sum_{\rho \in \mathfrak{S}(D)} F\left(\sigma\left(Z_{\mu(1)}\right), \rho\left(Z_{\mu(2)}\right), \tau \varepsilon\right) \\
= & \sum_{\tau \in \mathbb{Z}_{2}} \sum_{\sigma \in \mathfrak{S}(D)} \sum_{\rho \in \mathfrak{S}(D)}\left\{F\left(\sigma\left(Z_{1}\right), \rho\left(Z_{2}\right), \tau \varepsilon\right)+F\left(\sigma\left(Z_{2}\right), \rho\left(Z_{1}\right), \tau \varepsilon\right)\right\} \\
= & 2 c(D) \cdot(D !)^{2} \cdot \varepsilon \cdot\left(\mathrm{e}^{\varepsilon\left|Z_{1}\right|-\left|Z_{2}\right|}+\mathrm{e}^{\varepsilon\left|Z_{2}\right|-\left|Z_{1}\right|}\right. \\
= & \varepsilon\left(\mathrm{e}^{-\left|Z_{1}\right|} \sinh \left|\mathrm{e}_{2}\right|+\mathrm{e}^{-\left|Z_{2}\right|} \sinh \left|Z_{1}\right|-\left|Z_{2}\right|\right) .
\end{aligned}
$$

We have used the invariance under the action of two copies $\mathfrak{S}(D)$, which contributed a factor $(D !)^{2}$.

\subsection{Multivariable Graph Calculus}

Let $\mathfrak{h}=\left\{h_{1} \ldots, h_{n}\right\} \subset \mathrm{G}_{D}$ be a set of connected, non-isomorphic graphs. For the basis of the multivariable calculus the free monoid $\operatorname{FM}\left(\left\{h_{1}, \ldots, h_{n}\right\}\right)$ is too 'verbose', and not each one of its elements has the ordered form $h_{1}^{\alpha_{1}} \cdots h_{n}^{\alpha_{n}}$. This could in principle be solved by taking the free commutative monoid $\mathrm{FM}^{\mathrm{ab}}(\mathfrak{h})$ instead, which, however, tuns out to be overly restrictive (for our aims). A mild compromise between these two alternatives - the free monoid and its abelianisation - is to allow to permute letters in an arbitrary word, as to make use of the action (2.11), and then in some sense undo the changes. Next definition introduces precisely such reordering.

Definition 3 Given a finite set of graphs $\mathfrak{h}=\left\{h_{1}, \ldots, h_{n}\right\}$, the degree $|g|$ of an element $g$ in $\operatorname{FM}(\mathfrak{h})$ is the number of factors of $g$, i.e. the number of connected components $g$ consists of. We let $\mathfrak{S}(|g|)$ act by permuting the factors of $g, g \mapsto$ $\sigma(g)$; notice that $\mathfrak{S}(|g|)$ left-acts naturally as $\sigma \cdot f=f \circ \sigma^{-1}$ on functions $f$ : 
$\mathscr{N}(g) \rightarrow \mathbb{C}$. Given a family of functions $\left\{u_{g}\right\}_{g \in H}$ supported on a system of graphgroup actions $\{\mathscr{N}(g), G(g)\}_{g \in H \subset \mathrm{FM}(\mathfrak{h})}$, and given a $g \in H$, we declare the pairs $\left(u_{g}, g\right) \sim\left(\sigma \cdot u_{g}, \sigma(g)\right)$ equivalent for each $\sigma \in \mathfrak{S}(|g|)$. The notation we choose for this equivalence, called reordering, is

$$
u_{g} g \sim u_{h} h \quad \text { if and only if } \quad u_{h}=\sigma \cdot u_{g} \text { and } h=\sigma(g) \text { for certain } \sigma \in \mathfrak{S}(|g|) .
$$

Definition 4 Given a finite set $\mathfrak{h}=\left\{h_{1}, \ldots, h_{n}\right\} \subset \mathrm{G}_{D}$ of connected nonisomorphic graphs, a system of graph-group actions $\mathcal{S}=\{\mathscr{V}(h), G(h)\}_{h \in \mathfrak{h}}$ is said to be independent if (2.10) holds. When the context is clear, we just say that ' $h$ is independent', or that $\mathcal{S}$ is.

Definition 5 A multivariable graph calculus $\mathscr{C}(\mathfrak{h})$ or a graph calculus with variables $\mathfrak{h}$ consists of two objects:

- the choice of an independent system of graph-group actions $\{\mathscr{V}(h), G(h)\}_{h \in \mathfrak{h}}$ for a finite set $\mathfrak{h} \subset \mathrm{G}_{D}$ and

- the set of finite formal sums in elements of $g \in \mathrm{FM}(\mathfrak{h})$ having each of these a function of the form $v_{g}: \mathscr{N}(g) \rightarrow \mathbb{C}$ as coefficient, modulo reordering. That is,

$$
\mathscr{C}(\mathfrak{h})=\left\{\sum_{g} v_{g} g \mid v_{g} \equiv 0 \text { for almost all } g \in \mathrm{FM}(\mathfrak{h})\right\} / \sim
$$

where $\sim$ is the linear extension of relation (2.12), abusing on the same symbol.

\subsection{Algebraic Structure}

We now explore the structure of a graph calculus $\mathscr{C}(\mathfrak{h})$ with variables $\mathfrak{h}=$ $\left\{h_{1}, \ldots, h_{n}\right\}$. The elements of $\mathscr{C}(\mathfrak{h})$, called also functionals, have a non-unique representation, since $\sum_{g} v_{g} g=\sum_{\tilde{g}} v_{\tilde{g}} \tilde{g}$ where $\tilde{g}=\tau_{g}(g)$ and $v_{\tilde{g}}=\tau_{g} \cdot\left(v_{g}\right)$ for an arbitrary $\tau_{g} \in \mathfrak{S}(|g|)$. For sake of computability, it will be helpful to be able to fix representing elements $g$ that span a functional, and subordinate the order of the arguments of the coefficient-functions to that choice.

We write $g \stackrel{\mathrm{c}}{\sim} h$ for any $g, h \in \mathrm{FM}(\mathfrak{h})$ if $g=h$ in the free commutative monoid $\operatorname{FM}^{\mathrm{ab}}(\mathfrak{h})$ spanned by $\mathfrak{h}$. In other words, $g \underset{\mathrm{c}}{\sim} h$ if and only if $h$ and $g$ match in $\operatorname{FM}(\mathfrak{h})$ up to a rearranging $\sigma \in \mathfrak{S}(|g|)$, i.e. if $\sigma(g)=h$.

Definition 6 Given a family of functions $\left\{v_{l}\right\}_{l}$ supported on $\{\mathscr{V}(l), G(l)\}_{l \in H}$ where $H \subset \operatorname{FM}(\mathfrak{h})$. Let $g, h \in H$ be such that

$h \underset{\mathrm{c}}{\sim} g$. We define for the reordering of a function $v_{h}: \mathscr{N}(h) \rightarrow \mathbb{C}$ with respect to $g$ by

$$
\Theta_{g}\left(v_{h}\right)=\sigma \cdot v_{g}, \text { if } \sigma(g)=h \text { as elements of } \operatorname{FM}(\mathfrak{h}),
$$

being $\sigma$ the rearranging element $\sigma \in \mathfrak{S}(|g|)=\mathfrak{S}(|h|)$.

We shall drop the subindex $g$ in $\Theta_{g}(\bullet)$ when the context is clear. If one factors $g$ as $g_{1} g_{2}$ with respect to an 'abelianised' product, an element $\sigma \in \mathfrak{S}(|g|)$ serves as correction, so that $\sigma\left(g_{1} g_{2}\right)=g$. Their rearranging yields $\Theta_{g}\left(u_{g_{1}} t_{g_{2}}\right)=\sigma \cdot\left(u_{g_{1}} t_{g_{2}}\right)$ for 
suitably chosen functions $u_{g_{1}}$ and $t_{g_{2}}$. In general, the collection of graphs $g_{1}, \ldots, g_{r}$ is not required to be connected. If the context is clear, we pick this rearranging element in a smaller group $\sigma \in \mathfrak{S}(r)$ that only permutes the arguments of $\mathscr{V}\left(g_{i}\right)$.

Definition 7 Denote by $H_{1} H_{2}$ the subset $\left\{g_{1} g_{2} \mid g_{a} \in H_{a}\right\}$ in the free commutative monoid $\mathrm{FM}^{\mathrm{ab}}(\mathfrak{h})$ spanned by an independent set of graphs $\mathfrak{h}$. Given two functionals in $\mathscr{C}(\mathfrak{h}), U=\sum_{h \in H_{1}} u_{h} h$ and $T=\sum_{h \in H_{2}} t_{h} h$, we define their product $V=(U \cdot T)$ as the functional

$$
V=U \cdot T=\sum_{g \in H_{1} H_{2} \subset \mathrm{FM}^{\mathrm{ab}}(\mathfrak{h})} v_{g} g,
$$

whose coefficients $v_{g}$ are given by the 'ordered convolution'

$$
v_{g}=\sum_{\substack{\left(g_{1}, g_{2}\right) \in H_{1} \amalg H_{2} \\ g_{1} g_{2} \sim \mathrm{c} g}} \Theta_{g}\left(u_{g_{1}} t_{g_{2}}\right) \text {. }
$$

Lemma 1 This product on $\mathscr{C}(\mathfrak{h})$ is commutative.

Proof Let $U=\sum_{j \in J} u_{j} j$ and $T=\sum_{l \in L} t_{l} l$ be in $\mathscr{C}(\mathfrak{h})$. Given $g \in \operatorname{FM}(\mathfrak{h})$ with $g \stackrel{\mathrm{c}}{\sim} j l$ for some $j \in J$ and some $l \in L$ we show that the components $v_{g}$ of $V=U \cdot T$ and $\tilde{v}_{g}$ of $\tilde{V}=T \cdot U$ satisfy $v_{g} g=\tilde{v}_{g} g$ in $\mathscr{C}(\mathfrak{h})$. It suffices to exhibit an element $v \in$ $\mathfrak{S}(|g|)$ that satisfies $\Theta_{g}\left(u_{j} t_{l}\right) g=\Theta_{g}\left(t_{l} u_{j}\right) v(g)$. This $v$ will be next constructed.

We have the freedom to assume that $g=h_{1}^{\alpha_{1}} \cdots h_{n}^{\alpha_{n}}$. Since $j, l \in \operatorname{FM}(\mathfrak{h})$,

$$
j \underset{\mathrm{c}}{\sim} h_{1}^{\theta_{1}} h_{2}^{\theta_{2}} \cdots h_{n}^{\theta_{n}} \quad \text { and } \quad l \underset{\mathrm{c}}{\sim} h_{1}^{\lambda_{1}} h_{2}^{\lambda_{2}} \cdots h_{n}^{\lambda_{n}}
$$

for some $0 \leq \lambda_{i}, \theta_{i} \leq \alpha_{i}$ that satisfy $\alpha_{i}=\theta_{i}+\lambda_{i}, i=1, \ldots, n$. In the notation introduced above, \# $I\left(j, h_{i}\right)=\theta_{i}$ and \# $I\left(l, h_{i}\right)=\lambda_{i}$. We begin by assuming that the relations above are equalities,

$$
j=h_{1}^{\theta_{1}} h_{2}^{\theta_{2}} \cdots h_{n}^{\theta_{n}} \quad \text { and } \quad l=h_{1}^{\lambda_{1}} h_{2}^{\lambda_{2}} \cdots h_{n}^{\lambda_{n}},
$$

and restore towards the end the more general form (2.15). Let $|j|=\theta_{1}+\ldots+\theta_{n}$ and $|l|=\lambda_{1}+\ldots+\lambda_{n}$ be the orders of $j$ and $l$. We define first $\sigma \in \mathfrak{S}(|g|)$ as the $(|j|,|l|)$-shuffle determined by

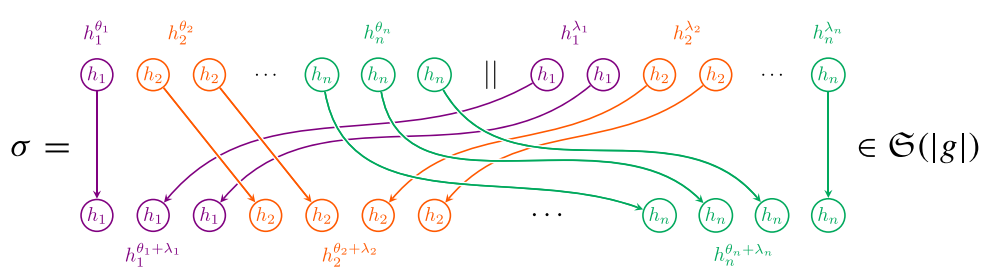

Each $h_{i}$ before double bar in the first row is a factor of $j$; after the double bar, the $h_{i}$ 's represent the factors of $l$. The lower is a factorisation of $g$. Thus the diagram states that $\sigma(j l)=g$. Analogously, we can define a $(|l|,|j|)$-shuffle $\rho$ that satisfies $\rho(l j)=g$. This is depicted in the following diagram, in which we represent $l$ to the left of the double bar and $j$ to the right. 


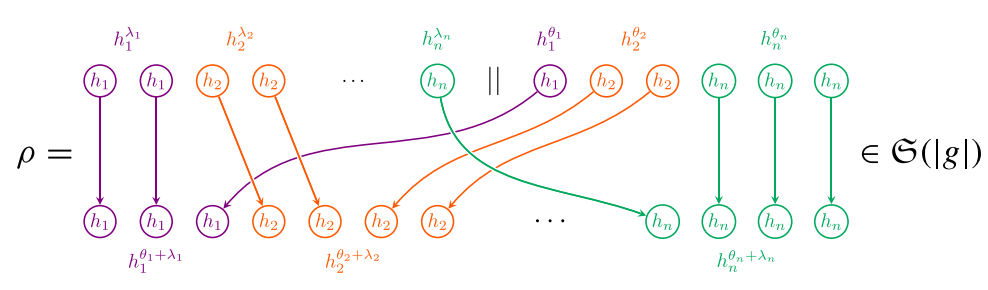

One has $\rho(l j)=g=\sigma(j l)$ but this still does not guarantee that $\rho^{-1} \sigma\left(v_{j l}\right)=\tilde{v}_{l g}$. In order to correct this, we define certain permutations $\tau_{i}$ that are constant everywhere except in the elements pertaining a particular $h_{i}$ for fixed $i$. This embeds $\mathfrak{S}\left(\# I\left(g ; h_{i}\right)\right) \subset \mathfrak{S}(|g|)$. Notice first that for each such element $\mu \in \mathfrak{S}\left(\# I\left(g ; h_{i}\right)\right)$

$$
w_{g} g=\left(\mu \cdot w_{g}\right) \mu(g)=\left(\mu \cdot w_{g}\right) g
$$

holds in $\mathscr{C}(\mathfrak{h})$ for any $w_{g}: \mathscr{N}(g) \rightarrow \mathbb{C}$. For each $i=1, \ldots, n$, define $\tau_{i}$ as certain permutation $\beta_{i}$ (given below) in the range $\left[\alpha_{i-1}+1, \alpha_{i}\right]$ and constant outside it:

$$
\tau_{i}(x)=\left\{\begin{array}{ll}
\beta_{i}(x) & 0<x-s_{i}<\alpha_{i} \\
x & \text { otherwise }
\end{array},\right.
$$

where $s_{i}:=\left(\alpha_{1}+\ldots+\alpha_{i-1}\right)$

$$
\beta_{i}(x)= \begin{cases}x+\lambda_{i} & s_{i}<x \leq s_{i}+\theta_{i}, \\ x-\theta_{i} & \theta_{i}<x \leq \theta_{i}+\lambda_{i}=\alpha_{i} .\end{cases}
$$

Then the sought-after $v$ is

$$
v=\rho^{-1} \circ\left(\tau_{1} \circ \cdots \circ \tau_{n}\right) \circ \sigma,
$$

which by construction satisfies

$$
\left(v \cdot v_{j l}\right) v(j l)=\tilde{v}_{l j} l j .
$$

The map $v$ is thus given by

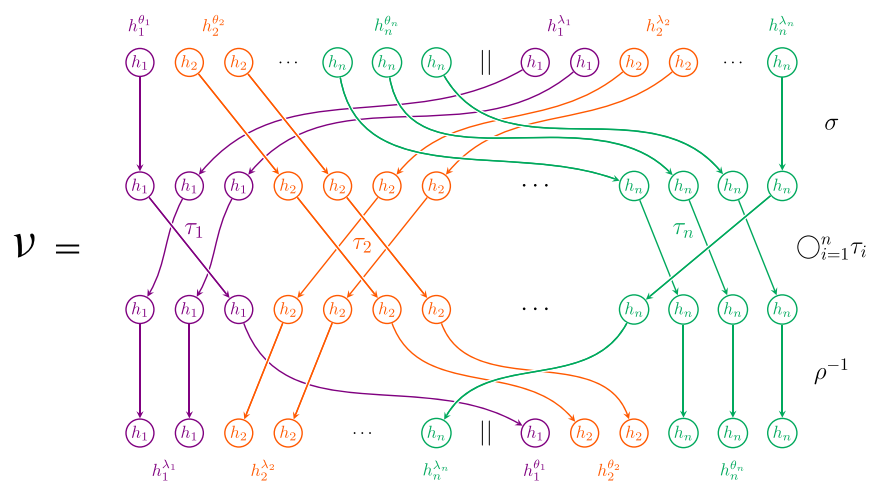

We now come back to the strongest, original statement, in which $l$ and $j$ have the form (2.15), instead of (2.16). This means that there are permutations $\gamma \in \mathfrak{S}(|j|) \subset$ $\mathfrak{S}(|j|+|l|)$ and $\delta \in \mathfrak{S}(|l|) \subset \mathfrak{S}(|j|+|l|)$ with

$$
\gamma(j)=h_{1}^{\theta_{1}} h_{2}^{\theta_{2}} \cdots h_{n}^{\theta_{n}} \quad \text { and } \quad \delta(l)=h_{1}^{\lambda_{1}} h_{2}^{\lambda_{2}} \cdots h_{n}^{\lambda_{n}} .
$$


Then we correct $v$ by these two elements:

$$
v=\rho^{-1} \circ\left(\tau_{1} \circ \cdots \circ \tau_{n}\right) \circ \sigma \circ(\gamma, \delta)
$$

which satisfies, in the most general case, (2.17). The statement follows by linear extension of it.

Example 4 To illustrate this notation, consider the sets $H=\left\{f g, f^{2}\right\}$ and $K=$ $\left\{f g, g^{2}\right\}$ of coloured graphs and let $U=\sum_{e \in K} u_{e} e$ and $T=\sum_{e \in H} t_{e} e$. If $X_{A}$ are momenta of $f$ and $Z_{A}$ of $g$ (for $A=1,2$ ), we pick a particular graph $l=f^{2} g^{2}$, and define the following permutations in $\mathfrak{S}(4)$

$$
\sigma=(13)(24)=\{\tau=(1)(23)(4)=\mid\}
$$

The coefficient $v_{l}=v_{f^{2} g^{2}}$ is given by

$$
\begin{aligned}
v_{f^{2} g^{2}}\left(X_{1}, X_{2}, Z_{1}, Z_{2}\right)= & \left(\Theta_{l}\left(u_{f g} t_{f g}\right)+\Theta_{l}\left(u_{g^{2}} t_{f^{2}}\right)\right)\left(X_{1}, X_{2}, Z_{1}, Z_{2}\right) \\
= & \left(\tau \cdot\left(u_{f g} t_{f g}\right)\right)\left(X_{1}, X_{2}, Z_{1}, Z_{2}\right) \\
& \quad+\left(\sigma \cdot\left(u_{g^{2}} t_{f^{2}}\right)\right)\left(X_{1}, X_{2}, Z_{1}, Z_{2}\right) \\
= & u_{f g} t_{f g}\left(X_{1}, Z_{1}, X_{2}, Z_{2}\right)+u_{g^{2}} t_{f^{2}}\left(Z_{1}, Z_{2}, X_{1}, X_{2}\right) .
\end{aligned}
$$

Structures appearing in the graph calculus resemble the monoid ring. Given a commutative unit ring $R$ and a monoid $M$, the monoid ring [38, Ch. II] is built by formal finite sums in $M$ with coefficients in $R$,

$$
R[M]=\left\{\sum_{m} r_{m} m \mid r_{m} \in R, m \in M \& r_{n} \neq 0 \text { for finitely many } n \in M\right\},
$$

and endowed with the convolution product. The structure of the graph calculus generated by $n$ variables $\mathfrak{h}=\left\{h_{1}, \ldots, h_{n}\right\}$ requires to define, instead of the ring $R$, the collection $\mathcal{A}$ of algebras of functions

$$
\mathcal{A}=\prod_{g \in \mathrm{FM}(\mathfrak{h})} A_{g}, \quad \text { where } A_{g}=\{\text { functions } \mathscr{V}(g) \rightarrow \mathbb{C}\},
$$

and then to consider the following restricted version of the monoid algebra

$$
\mathcal{A}[\operatorname{FM}(\mathfrak{h})]:=\left\{\sum_{g \in \operatorname{FM}(\mathfrak{h})} u_{g} g \mid u_{g} \in A_{g}, u_{h}=0 \text { for almost all } h \in \operatorname{FM}(\mathfrak{h})\right\} .
$$

Then we see that

$$
\mathscr{C}(\mathfrak{h})=\mathcal{A}[\mathrm{FM}(\mathfrak{h})] / \sim .
$$

Given functionals $U=\sum_{h \in H} u_{h} h, T=\sum_{l \in L} t_{l} l$ in $\mathscr{C}(\mathfrak{h})$ one defines their sum by

$$
U+T=\sum_{m \in H \cup L}\left(\sum_{\substack{\mathrm{c} \\ h \sim m, h \in H}} \Theta_{m}\left(u_{h}\right)+\sum_{\substack{\mathrm{c} \\ l \sim m, l \in L}} \Theta_{m}\left(t_{n}\right)\right) m ;
$$

for a scalar $x \in \mathbb{C}$, the functional $x U$ is defined by componentwise multiplication by $x$,

$$
x U=\sum_{h \in H}\left(x \cdot u_{h}\right) h
$$


The in-depth study of the structure of $\mathscr{C}(\mathfrak{h})$ is beyond the scope of this paper. Here, we will prove the properties that are useful to in later sections.

We observe that the Borel transform $\mathrm{B} A(j)$ of a series $A(j)=\sum_{n} a_{n} j^{n}$, given by $\mathrm{B} A(j)=\sum_{n}\left(a_{n} / n !\right) j^{n}$, can be seen as the rescaling of the coefficients of each $j^{n}$ by the inverse of the symmetry factors $n !=\# \mathfrak{S}(n)=\# \operatorname{Aut}(*)$ of the $n$-valent graph $*$. The association with a graph comes from the ordinary field theory, where * represents the $n$-point function. The series $A(j)$ are promoted to functionals $A[J]$ in a source $J$, now a function, and the coefficients of $J^{n} / \#$ Aut(*) are the correlation functions. This inspires (although this will be clear only after Section 3 ) the terminology of next definition. We warn that the name 'Borel' is purely formal and should not evoke any transfer of convergence properties from the ordinary to the colored case:

Definition 8 (Coloured Borel transform) Let $V=\sum_{h \in H} v_{g} g$ be a generating functional of graph-group actions. Then define its coloured Borel transform by

$$
\mathrm{B}_{\mathrm{c}}(V):=\sum_{g \in H \subset \mathrm{G}_{D}} \frac{1}{|G(g)|} v_{g} g \text {. }
$$

If $H \subset \mathrm{G}_{D} \ni h$, the following set

$$
H_{h}:=\left\{g \in \mathrm{G}_{D} \mid h g \stackrel{\mathrm{c}}{\sim} j \text { for some element } j \text { of } H\right\}
$$

will be relevant for the next lemma.

Lemma 2 Consider the generating functional of a system of graph-group actions $V=\sum_{g \in H} v_{g} g$ belonging to a graph calculus in (connected graph) variables $\mathfrak{h}=$ $\left\{h_{1}, \ldots, h_{n}\right\} \subset \mathrm{G}_{D}$. Suppose that the coefficients $v_{c_{1} \cdots c_{a} \cdots c_{b} \cdots c_{p}}$ obey the following rule under the transposition $(a b) \in \mathfrak{S}(p)$ of graphs:

$$
v_{c_{1} \ldots c_{a} \ldots c_{b} \ldots c_{p}}=(a b) \cdot v_{c_{1} \ldots c_{b} \ldots c_{a} \ldots c_{p}}
$$

for each $c_{a}, c_{b} \in \mathfrak{h}$, where $a$ and $b$ denote the number of factor (connected component) where $c_{a}$ and $c_{b}$ are located, respectively. Moreover, assume that $v_{c_{1} \cdots c_{r} \cdots c_{p}}$ is invariant under $G\left(c_{r}\right)$ for each factor $c_{r}, 1 \leq r \leq p$. Then, one has for $h=c_{r}$,

$$
\frac{\delta \mathrm{B}_{\mathrm{c}}(V)}{\delta h}=\sum_{g \in H_{h}} \frac{1}{|G(g)|} v_{h g} g
$$

which means that for each $X \in \mathscr{V}(h)$,

$$
\frac{\delta \mathrm{B}_{\mathrm{c}}(V)}{\delta h(X)}=\sum_{g \in H_{h}} \frac{1}{|G(g)|}\left(\iota_{X}^{1} v_{h g}\right) g=\sum_{g \in H_{h}} \frac{1}{|G(g)|} v_{h g}(X, \bullet) g,
$$

being $\iota_{X}^{1}$ the insertion of momentum $X$ of $h$ at the first argument of the function $v_{\text {hg }}$.

Proof By assumption, one can bring every element $g \in H$ to the form $g=$ $h_{1}^{\alpha_{1}} \cdots h_{n}^{\alpha_{n}}$, and change the coefficients $v_{g}$ accordingly without alteration. Notice that 
each $g \in H$ can be further factorised as $h^{\ell} f$, where $\delta f / \delta h \equiv 0$, and $\ell$ depends on $g$. Through direct computation,

$$
\begin{aligned}
\frac{\delta \mathrm{B}_{\mathrm{c}}(V)}{\delta h(X)} & =\sum_{g=h^{\ell} f \in H} \sum_{r=1, \ldots, \ell} \sum_{\sigma \in G(h)} \frac{1}{\left|G^{\prime}\left(h^{\ell} f\right)\right|} \iota_{\sigma(X)}^{r} v_{h^{\ell} f} h^{\ell-1} f \\
& =\sum_{g=h^{\ell} f \in H} \sum_{\sigma \in G(h)} \frac{\ell}{\left|G\left(h^{\ell} f\right)\right|} \iota_{\sigma(X)}^{1} v_{h^{\ell} f} h^{\ell-1} f \\
& =\sum_{g=h^{\ell} f \in H} \frac{\ell \cdot|G(h)|}{\left|G\left(h^{\ell} f\right)\right|} \iota_{X}^{1} v_{h^{\ell} f} h^{\ell-1} f .
\end{aligned}
$$

First, we used the invariance (2.19), i.e. $\iota_{X}^{m} v_{h^{\ell} f}=\iota_{X}^{p} v_{h^{\ell} f}$ for each $1 \leq p, m \leq \ell$; then, the invariance under $G(h)$. Throughout, we can assume $\ell \geq 1$, since this is required for a summand in the first equality of (2.20) not to vanish. Also, since

$$
G\left(h^{\ell} f\right)=G\left(h^{\ell}\right) \imath \mathfrak{S}(\ell) \times G(f),
$$

the orders of the groups should satisfy

$$
\left|G\left(h^{\ell} f\right)\right|=\ell ! \cdot|G(h)|^{\ell} \cdot|G(f)|=\ell|G(h)| \cdot\left|G\left(h^{\ell-1} f\right)\right| .
$$

Hence, after cancellation one gets

$$
\frac{\delta \mathrm{B}_{\mathrm{c}}(V)}{\delta h(X)}=\sum_{g \in H_{h}} \frac{1}{|G(g)|} \iota_{X}^{1} v_{h g} g .
$$

Definition 9 The graph derivative $\partial U / \partial h$ of a generating functional of group actions $U$ is given by the coefficient $v_{\varnothing}$ of the empty graph $\varnothing$ of the functional derivative of $U$ with respect to $h, \delta U / \delta h=: \sum_{g} v_{g} g$, to wit

$$
\frac{\partial U}{\partial h}=\left(\frac{\delta U}{\delta h}\right)_{\varnothing} .
$$

Parenthetically, the difference in notations for 'partial derivative' and 'functional derivative' does not intend to mirror any difference between multivariable ordinary and functional calculi.

The next result is simple and useful at the same time:

Lemma 3 (Graph calculus Leibniz product rule) Consider a graph calculus $\mathscr{C}(\mathfrak{h})$ and let $J, L \subset \mathrm{FM}(\mathfrak{h})$ span functionals $U$ and $T$ in $\mathscr{C}(\mathfrak{h})$,

$$
U=\sum_{j \in J} u_{j} j, \quad T=\sum_{l \in L} t_{l} l
$$

Then the graph derivative of the product $V=U \cdot T=\sum_{g} v_{g} g$ is

$$
\frac{\partial(U \cdot T)}{\partial g}=\sum_{\Omega \in G(g)} \Omega \cdot v_{g}=\sum_{\Omega \in G(g)} \sum_{\substack{j, l) \in J \amalg L \\ j l \sim g}} \Omega \cdot \Theta_{g}\left(u_{j} t_{l}\right) .
$$


Proof We compute directly the derivative of the product $U \cdot T$ with respect to $g$ from the $\varnothing$-coefficient of $\delta(U \cdot T) / \delta g$ :

$$
\begin{aligned}
& \frac{\partial(U \cdot T)}{\partial g}=\left(\frac{\delta(U \cdot T)}{\delta g}\right)_{\varnothing} \\
& =\left(\frac{\delta}{\delta g} \sum_{f \in J L \subset \mathrm{FM}^{\mathrm{ab}}(\mathfrak{h})} \sum_{\substack{j, l) \in J \amalg L \\
j l \sim f}} \Theta_{f}\left(u_{j} t_{l}\right) f\right)_{\varnothing} \\
& =\sum_{f \in J L \subset \mathrm{FM}^{\mathrm{ab}}(\mathfrak{h})} \frac{\delta f}{\delta g} \sum_{\substack{j, l) \in J \amalg L \\
j l \sim f}} \Theta_{f}\left(u_{j} t_{l}\right) \\
& =G(g) \curvearrowright\left[\sum_{\substack{(j, l) \in J \amalg L \\
j l \sim g}} \Theta_{g}\left(u_{j} t_{l}\right)\right] \\
& =\sum_{\Omega \in G(g)} \sum_{\substack{j, l) \in J \amalg L \\
j l \sim g \\
\mathrm{c} g}} \Omega \cdot \Theta_{g}\left(u_{j} t_{l}\right) .
\end{aligned}
$$

For the second equality we inserted the coefficients explicitly, according to the definition of the product. The fourth equality holds by graph independence, (2.10), guaranteed for a graph calculus.

From now on let $2 k(g)=\left|g^{0}\right|$ denote the number of vertices of $g \in \mathrm{G}_{D}$. Our canonical example of system of graph-group actions have the form

$$
\left\{u_{g}, \mathscr{N}(g)=M_{D \times k(g)}(\mathbb{Z}), G(g)=\operatorname{Aut}_{\mathrm{c}}(g)\right\}_{g \in H} .
$$

Due to the rigidity of a coloured graph, each automorphism ${ }^{9} \pi$ of a connected graph $g$ is determined by a permutation $\sigma$ of the black (or white) vertices of $g$; we write $\pi=\hat{\sigma}$ for such $\sigma \in \mathfrak{S}(k(g))$. The action of the coloured automorphisms $\hat{\sigma} \in$ Aut $_{\mathrm{c}}(g) \subset \mathfrak{S}(k(g))$ on $M_{D \times k(g)}(\mathbb{Z})$ is by permutation of the matrix columns, $\mathbf{y}^{i} \mapsto$ $\mathbf{y}^{\sigma(i)}, i=1, \ldots, k$. As a notational remark, we will often write $k$ instead of $k(g)$, as we just did, if the context is clear.

\subsection{Three Limit Cases and Examples}

The previous lemma implies the Leibniz multivariable rule. Before elaborating on it, for the case of graph-group actions by automorphisms, $G(g)=\operatorname{Aut}_{\mathrm{c}}(g)$, it will be helpful to exhibit this group action on a function $v_{g}$ in three limit cases, according to the graph type of $g$.

\footnotetext{
${ }^{9}$ There are more than one definition of 'automorphism of a coloured graph'. The one used here is introduced in [53]. In this setting, an automorphism of a coloured graph is a graph-morphism that preserves the colouring of the edges and bipartiteness of the vertex-set in a strict way (not up to a permutation of colours as the factor $1 / 3$ in the action of the quartic rank-3 model in [20] suggests). That is, edges of colour $a$ have to be mapped to edges of colour $a$; black (resp. white) vertices to black (resp. white) vertices.
} 
Consider $\left\{\mathscr{V}(g), \operatorname{Aut}_{\mathrm{c}}(g)\right\}_{g \in \mathfrak{h}}$, an independent system graph-group actions, $\mathfrak{h}=$ $\left\{h_{1}, \ldots, h_{n}\right\} \subset \mathrm{G}_{D}$. Let $g=h_{1}^{\alpha_{1}} \cdots h_{n}^{\alpha_{n}}$ and let $U$ and $T$ graph-generated functionals by $J$ and $L$, respectively, ${ }^{10}$ being these subsets of the the monoid generated by $\left\{h_{1}, \ldots, h_{n}\right\}$. Then according to Lemma 3 ,

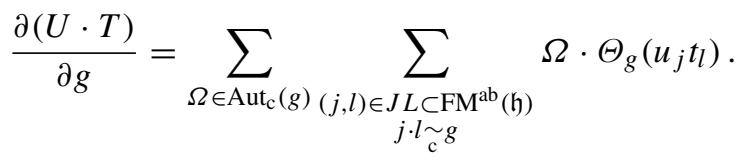

This relation holds in a subdomain $\mathscr{U}(g)=\mathcal{F}_{D, k(g)} \subset \mathscr{N}(g)=M_{D \times k(g)(\mathbb{Z})}$ to be justified later (see Section 3.2):

$$
\begin{array}{r}
\mathcal{F}_{D, k}:=\left\{\left(\mathbf{y}^{1}, \ldots, \mathbf{y}^{k}\right) \in M_{D \times k}(\mathbb{Z}) \mid \quad y_{c}^{\alpha} \neq y_{c}^{v} \text { for all } c=1, \ldots, D\right. \\
\text { and } \alpha, v=1, \ldots, k, \alpha \neq v\} .
\end{array}
$$

The canonical action of $\operatorname{Aut}_{\mathrm{c}}(g)$ obviously restricts to this set $\mathscr{U}(g)=\mathcal{F}_{D, k(g)}$. Recalling that $\operatorname{Aut}_{\mathrm{c}}(g)=\operatorname{Aut}_{\mathrm{c}}\left(h_{1}^{\alpha_{1}} \amalg h_{2}^{\alpha_{2}} \amalg \ldots \amalg h_{n}^{\alpha_{n}}\right)=\prod_{i=1}^{n} \operatorname{Aut}_{\mathrm{c}}\left(h_{i}\right)_{2} \mathfrak{S}\left(\alpha_{i}\right)$, first we elaborate on three simple cases:

- Case I: if $n=1$. Then $g=h^{\alpha}$, any $\Omega \in \operatorname{Aut}_{\mathrm{c}}(g)=\operatorname{Aut}_{\mathrm{c}}(h)$ ᄀ $\mathfrak{S}(\alpha)$ is given by $\sigma=\left(\sigma^{1}, \ldots, \sigma^{\alpha}\right) \in \operatorname{Aut}_{\mathrm{c}}(h)^{\alpha}$ and $\mu \in \mathfrak{S}(\alpha)$, yielding for (2.23)

$$
\Omega^{-1} \cdot\left(v_{g}\right)\left(X_{1}, \ldots, X_{\alpha}\right)=v_{g}\left(\sigma^{1} X_{\mu(1)}, \ldots, \sigma^{\alpha} X_{\mu(\alpha)}\right) \text {. }
$$

Here each $X_{A}=\left(\mathbf{x}_{A}^{1}, \ldots, \mathbf{x}_{A}^{k}\right) \in \mathcal{F}_{D, k(h)}$ and the action of the automorphism group $\tau \in \operatorname{Aut}_{\mathrm{c}}(h)$ is given by $\tau\left(X_{A}\right)=\left(\mathbf{x}_{A}^{\tau(1)}, \ldots, \mathbf{x}_{A}^{\tau(k)}\right)$.

- Case II: if $n \neq 1$ but $\alpha_{A}=1$ for all $A=1, \ldots, n$. In this case, $g=h_{1} h_{2} \cdots h_{n}$. Then $\operatorname{Aut}_{\mathrm{c}}(g)=\prod_{i} \operatorname{Aut}_{\mathrm{c}}\left(h_{i}\right) \ni \Omega=\left(\sigma_{1}, \ldots, \sigma_{n}\right)$, which acts like

$$
\Omega^{-1} \cdot\left(v_{g}\right)\left(X^{1}, \ldots, X^{n}\right)=v_{g}\left(\sigma_{1}\left(X^{1}\right), \ldots, \sigma_{n}\left(X^{n}\right)\right) .
$$

- Case III: If $g=h_{1}^{\alpha} \cdots h_{n}^{\alpha_{n}}$, but all automorphisms $\operatorname{Aut}_{\mathrm{c}}\left(h_{i}\right)$ are trivial. Then

$$
\operatorname{Aut}_{\mathrm{c}}(g)=\prod_{i} \operatorname{Aut}_{\mathrm{c}}\left(h_{i}\right) \imath \mathfrak{S}\left(\alpha_{i}\right)=\mathfrak{S}\left(\alpha_{1}\right) \times \cdots \times \mathfrak{S}\left(\alpha_{n}\right) \ni\left(\mu_{1}, \ldots, \mu_{n}\right) .
$$

We use now multi-index notation $\boldsymbol{\alpha}=\left(\alpha_{1}, \ldots, \alpha_{n}\right)$ and $\boldsymbol{\gamma}=\left(\gamma_{1}, \ldots, \gamma_{n}\right)$ and abbreviate $u_{\gamma_{1}, \ldots, \gamma_{n}}=u_{h_{1}^{\gamma_{1}} \ldots h_{n}^{\gamma_{n}}}$, and similarly for $t_{\gamma_{1}, \ldots, \gamma_{n}}$. One can rewrite then

$$
\begin{aligned}
& \frac{\partial(U \cdot T)}{\partial g}=\sum_{\mu_{1} \in \mathfrak{S}\left(\alpha_{1}\right)} \cdots \sum_{\mu_{n} \in \mathfrak{S}\left(\alpha_{n}\right)} \sum_{\begin{array}{c}
\left(\gamma_{i}, v_{i}\right) \\
\gamma_{i}, v_{i} \geq 0 \\
\gamma_{i}=\nu_{i} \\
i=1, \ldots, n
\end{array}}\left(\mu_{1}, \ldots, \mu_{n}\right)^{*} \Theta_{g}\left(u_{\gamma_{1}, \ldots, \gamma_{n}} t_{v_{1}, \ldots, v_{n}}\right) \\
& =\sum_{\mu_{1} \in \mathfrak{S}_{\left(\alpha_{1}\right)}} \cdots \sum_{\mu_{n} \in \mathfrak{S}_{\left(\alpha_{n}\right)}} \\
& \sum_{\substack{0 \leq \gamma_{i} \leq \alpha_{i} \\
i=1, \ldots, n}}\left(\mu_{1}, \ldots, \mu_{n}\right)^{*} \Theta_{g}\left(u_{\gamma_{1}, \ldots, \gamma_{n}} t_{\alpha_{1}-\gamma_{1}, \ldots, \alpha_{n}-\gamma_{n}}\right)
\end{aligned}
$$

\footnotetext{
${ }^{10}$ Of course, one could just take the union of the both spanning sets of graphs, if they do would not a priori coincide.
} 
in multi-index notation as

$$
\frac{\partial(U \cdot T)}{\partial g}=\sum_{\boldsymbol{\mu} \in \prod_{i_{1}}^{n} \mathfrak{S}\left(\alpha_{i}\right)} \sum_{\boldsymbol{\gamma} \leq \boldsymbol{\alpha}}(\boldsymbol{\mu})^{*} \Theta_{g}\left(u_{\boldsymbol{\gamma}} t_{\boldsymbol{\alpha}-\boldsymbol{\gamma}}\right) .
$$

For constant functions $u_{\gamma_{1}, \ldots, \gamma_{n}}, t_{\gamma_{1}, \ldots, \gamma_{n}}$, this should reduce to the multivariable product formula. Indeed,

$$
\frac{\partial(U \cdot T)}{\partial g}=\sum_{\boldsymbol{\gamma} \leq \boldsymbol{\alpha}}\left(\begin{array}{l}
\alpha_{1} \\
\gamma_{1}
\end{array}\right) \cdots\left(\begin{array}{l}
\alpha_{n} \\
\gamma_{n}
\end{array}\right) u_{\boldsymbol{\gamma}} t_{\boldsymbol{\alpha}-\boldsymbol{\gamma}}
$$

which is just the Leibniz rule (1.1).

Conveniently, lower-case (super)indices $(i=1, \ldots, n)$ of momenta label the graph type, whereas upper-case (sub)indices indicate the number of copy $\left(A=1, \ldots, \alpha_{i}\right)$ of the $i$-th graph type.

We describe now the action of $\operatorname{Aut}_{\mathrm{c}}(g)$ on a general function $v_{g}: M_{D \times k(g)}(\mathbb{Z}) \rightarrow$ $\mathbb{C}$.

Let $\mathbf{X}=\left(\left(X_{1}^{1}, \ldots, X_{\alpha_{1}}^{1}\right) \ldots\left(X_{1}^{n}, \ldots, X_{\alpha_{n}}^{n}\right)\right) \in M_{D \times k(g)}(\mathbb{Z})$, being $X_{A}^{i} \in$ $M_{D \times k\left(h_{i}\right)}(\mathbb{Z})$ the momentum of the $A$-th copy of $h_{i}$, for $A=1, \ldots, \alpha_{i}$. Picking an element $\Omega=(\boldsymbol{\sigma}, \boldsymbol{\mu}) \in \operatorname{Aut}_{\mathrm{c}}(g)$, with

$$
\begin{aligned}
\left(\sigma_{1}, \ldots, \sigma_{n}\right) & \in \prod_{i=1}^{n} \operatorname{Aut}\left(h_{i}\right)^{\alpha_{i}}, \quad \sigma_{i}=\left(\sigma_{i}^{1}, \ldots, \sigma_{i}^{\alpha_{i}}\right), \\
\boldsymbol{\mu}=\left(\mu_{1}, \ldots, \mu_{n}\right) & \in \prod_{i=1}^{n} \mathfrak{S}\left(\alpha_{i}\right),
\end{aligned}
$$

the following holds:

$$
\left(\Omega^{-1} \cdot v_{g}\right)\left(X_{A}^{i}\right)=v_{g}\left(\left(\sigma_{i}^{A} X_{\mu_{i}(A)}^{i}\right)_{A}^{i}\right),
$$

which is short-hand notation for

$$
\begin{aligned}
& \Omega^{-1} \cdot v_{g}(\mathbf{X}) \\
= & v_{g}\left(\left(\sigma_{1}^{1} X_{\mu_{1}(1)}^{1}, \sigma_{1}^{2} X_{\mu_{1}(2)}^{1} \ldots, \sigma_{1}^{\alpha_{1}} X_{\mu_{1}\left(\alpha_{1}\right)}^{1}\right), \ldots,\left(\sigma_{n}^{1} X_{\mu_{n}(1)}^{n}, \ldots, \sigma_{n}^{\alpha_{n}} X_{\mu_{n}\left(\alpha_{n}\right)}^{n}\right)\right) .
\end{aligned}
$$

Usually, it is summed all over $\Omega \in \operatorname{Aut}_{\mathrm{c}}(g)$, which being a group, allows us to choose whether we put the inverse in (2.26).

Example 5 For $e=\ominus, f={ }_{11}$ and $g=\bowtie$, let the subsets

$$
H=\left\{\varnothing, e^{2}, f^{2}\right\} \quad \text { and } \quad L=\left\{e^{3} f, e^{2} g, e^{2} f^{2} g\right\}
$$

span the functionals $U=\sum_{h \in H} u_{h} h$ and $T=\sum_{l \in L} t_{l} l$, and consider their product $V=U \cdot T$. According to the action (2.26), one has the following formulae: 
- For $b=e^{5} f$

$$
\begin{aligned}
\frac{\partial V}{\partial b} & =\sum_{\Omega \in \operatorname{Aut}_{\mathrm{c}}(b)} \Omega \cdot \Theta_{g}\left(u_{h} t_{l}\right) \\
& =\sum_{\Omega \in \operatorname{Aut}_{\mathrm{c}}\left(e^{5} f\right)} \Omega \cdot \Theta_{g}\left(u _ { e ^ { 2 } t _ { e ^ { 3 } f } ) } ( \sigma , \epsilon ) \cdot \left(u_{\left.e^{2} t_{e^{3} f}\right),}\right.\right. \\
& =\sum_{(\sigma, \epsilon) \in \mathfrak{S}(5) \times \mathbb{Z}_{2}}
\end{aligned}
$$

since $\operatorname{Aut}_{\mathrm{c}}\left(e^{5} f\right)=\operatorname{Aut}_{\mathrm{c}}(1 \mathfrak{g} 1) \imath \mathfrak{S}(5) \times \operatorname{Aut}_{\mathrm{c}}(\ominus) \imath \mathfrak{S}(1)=\{1\} \prec \mathfrak{S}(5) \times \mathbb{Z}_{2} \imath\{1\}$. Also the ordering $\Theta_{g}$ is trivial. This in turn means that for the five momenta $X_{A} \in \mathcal{F}_{1, D=3}=\mathbb{Z}^{3}$ of $e^{5}$ and the momentum $Z=\left(\mathbf{z}^{1}, \mathbf{z}^{2}\right) \in \mathcal{F}_{2,3}$,

$$
\frac{\partial V}{\partial e^{5} \mid f}\left(X_{A}, Z\right)=\sum_{(\sigma, \epsilon) \in \mathfrak{S}(5) \times \mathbb{Z}_{2}}\left(u_{e^{2}} t_{e^{3} \mid f}\right)\left(X_{\sigma(A)}, \mathbf{z}^{\epsilon(1)}, \mathbf{z}^{\epsilon(2)}\right)
$$

in abstract notation, or displaying the graphs:

$$
\begin{aligned}
\frac{\partial V}{\left.\partial \ominus^{5}\right|_{1 g^{1}}}\left(X_{A}, Z\right)= & \sum_{(\sigma, \epsilon) \in \mathfrak{S}(5) \times \mathbb{Z}_{2}} u_{\ominus \mid \ominus}\left(X_{\sigma(1)}, X_{\sigma(2)}\right) \\
& \times t_{\left.\ominus|\ominus| \ominus\right|_{1 /{ }_{1} 1}}\left(X_{\sigma(3)}, X_{\sigma(4)}, X_{\sigma(5)}, \mathbf{z}^{\epsilon(1)}, \mathbf{z}^{\epsilon(2)}\right) .
\end{aligned}
$$

- For $b^{\prime}=e^{2} f^{2} g$

$$
\frac{\partial V}{\partial b^{\prime}}=\sum_{\Omega \in \operatorname{Aut}_{c}\left(e^{2} f^{2} g\right)} \Omega \cdot\left\langle u_{\varnothing} t_{e^{2} f^{2} g}\right\rangle+\Omega \cdot\left\langle u_{\left.f^{2} t_{e^{2} g}\right\rangle} .\right.
$$

For $X_{A}$ momenta of $e^{2}, Z_{A}$ momenta of $f^{2}$ and total momentum $\mathbf{X}=\left(X_{1}, X_{2}, Z_{1}, Z_{2}, W\right)$, one sums over elements $\Omega=$ $\left(\epsilon,\left(\sigma^{1}, \sigma^{2} ; \mu\right), \tau\right) \in \operatorname{Aut}_{\mathrm{c}}\left(e^{2} f^{2} g\right)=\mathfrak{S}(2) \times\left(\mathbb{Z}_{2} \imath \mathfrak{S}(2)\right) \times \mathbb{Z}_{3}$, which yields for $\left(\partial V / \partial b^{\prime}\right)(\mathbf{X})=\left(\partial V / \partial\left(\left.\ominus^{2}\right|_{1 g{ }_{1}}{ }^{2} \mid \bowtie\right)\right)(\mathbf{X})$ the expression

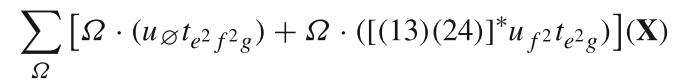

$$
\begin{aligned}
& =u_{\varnothing} \cdot\left\{\sum_{\left(\epsilon,\left(\sigma^{1}, \sigma^{2} ; \mu\right), \tau\right)}\left[t_{e^{2} f^{2} g}\left(X_{\epsilon(1)}, X_{\epsilon(2)}\right),\left(\sigma^{1} Z_{\mu(1)}, \sigma^{2} Z_{\mu(2)}, \tau(W)\right]\right\}\right. \\
& \left.\left.+\sum_{\left(\epsilon,\left(\sigma^{1}, \sigma^{2} ; \mu\right), \tau\right)} u_{f^{2}}\left(\sigma^{1} Z_{\mu(1)}, \sigma^{2} Z_{\mu(2)}\right) t_{e^{2} g}\left(X_{\epsilon(1)}, X_{\epsilon(2)}\right), \tau(W)\right)\right) \\
& =u_{\varnothing} \cdot\left\{\sum_{\left(\epsilon,\left(\sigma^{1}, \sigma^{2} ; \mu\right), \tau\right)}\left[t^{2}\left|g_{1}^{2}\right| \bowtie\left(X_{\epsilon(1)}, X_{\epsilon(2)}\right),\left(\sigma^{1} Z_{\mu(1)}, \sigma^{2} Z_{\mu(2)}\right), \tau(W)\right]\right\} \\
& \left.+\sum_{\left(\epsilon,\left(\sigma^{1}, \sigma^{2} ; \mu\right), \tau\right)} u_{1 \mathfrak{g}^{2} 2}\left(\sigma^{1} Z_{\mu(1)}, \sigma^{2} Z_{\mu(2)}\right) \times t_{\ominus^{2}}\left(X_{\epsilon(1)}, X_{\epsilon(2)}\right), \tau(W)\right) \text {. }
\end{aligned}
$$


One should still insert the explicit momenta $Z_{A}=\left(\mathbf{z}_{A}^{1}, \mathbf{z}_{A}^{2}\right) \in \mathcal{F}_{2,3}$, $W=\left(\mathbf{w}^{1}, \mathbf{w}^{2}, \mathbf{w}^{3}\right) \in \mathcal{F}_{3,3}, \tau(W)=\left(\mathbf{w}^{\tau(1)}, \mathbf{w}^{\tau(2)}, \mathbf{w}^{\tau(3)}\right)$, and $\sigma^{A} Z_{\mu(A)}=$ $\left(\mathbf{z}_{\mu(A)}^{\sigma^{A}(1)}, \mathbf{z}_{\mu(A)}^{\sigma^{A}(2)}\right)$.

\section{Tensor Models}

In this section, we implement the graph calculus for TFT.

\subsection{Tensor Field Theory}

The main idea to use a the Ward-identity [22] in order to decouple the SchwingerDyson equations (at a planar sector) and to obtain a master integral equation for the 2-point functions for matrix models [32] can been extended to the TFT setting. Some progress along these lines has been made for complex tensor field theory and consists in the further study [53] of the Ward-Takahashi identity of Ousmane-Samary [48] in order to descend the SDE tower [51] and eventually find closed equations. This led lately to the large- $N$ limit [50] of the connected- $\partial$ SDE. We treat a TFT as inspired by group field theory $[7,11,17,61]$.

Unlike matrix models, where there is a canonical way of forming a scalar, for tensor models a specific trace $\operatorname{Tr}_{\mathcal{B}}$, indicating how to contract the indices, should be specified. These traces $\operatorname{Tr}_{\mathcal{B}}$ are indexed by $D$-coloured graphs $\mathcal{B}$, where $D$ is the rank of the tensors $\varphi_{x_{1} \ldots x_{D}}, \bar{\varphi}_{x_{1} \ldots x_{D}}$.

The graphical representation of these traces derives from to the independence of the imposed transformation rules under the action of $\mathrm{U}(N)$ on the spaces corresponding to each index $x_{a}$ of $\varphi_{x_{1} \ldots x_{D}}, \bar{\varphi}_{x_{1} \ldots x_{D}}$, for $a=1, \ldots, D$, deemed colouring. That is to say, to form invariants only indices of identical colour can be contracted.

Therefore, a trace corresponding to a quartic interaction would be, say, 1 ig 1 formed by colour-wise contracting the indices with deltas, as follows:

$$
\operatorname{Tr}_{1 p_{1}}(\varphi, \bar{\varphi})=\sum_{\mathbf{x}, \mathbf{y}} \varphi_{x_{1} y_{2} x_{3}} \bar{\varphi}_{x_{1} y_{2} y_{3}} \varphi_{y_{1} y_{2} y_{3}} \bar{\varphi}_{y_{1} x_{2} x_{3}}
$$

The actual index-set of the tensors is $\{1, \ldots, N\}$, but thinking of $N$ as a large integer, we typically write these sums over $\mathbb{N}$ or $\mathbb{Z}$, or omit the domains in the sums. Although orthogonal groups [20], compact symplectic groups [18] and mixed symmetries [62] define other classes of tensor models, we restrict our discussion to the $\mathrm{U}(N)$-tensor models we just introduced.

A tensor model is thus determined by a dimension $D$ (the rank of the tensors) and an action $S[\varphi, \bar{\varphi}]$ given by a finite sum of traces indexed by connected $D$-coloured graphs. The partition function is given by

$$
\begin{aligned}
& Z[J, \bar{J}]=Z[0,0] \frac{\int \mathcal{D}[\varphi, \bar{\varphi}] \mathrm{e}^{\operatorname{Tr}(\bar{J} \varphi)+\operatorname{Tr}(\bar{\varphi} J)-N^{D-1} S[\varphi, \bar{\varphi}]}}{\int \mathcal{D}[\varphi, \bar{\varphi}] \mathrm{e}^{-N^{D-1} S[\varphi, \bar{\varphi}]}}, \\
& \mathcal{D}[\varphi, \bar{\varphi}]:=\prod_{\mathbf{x}} N^{D-1} \frac{\mathrm{d} \varphi_{\mathbf{x}} \mathrm{d} \bar{\varphi}_{\mathbf{x}}}{2 \pi \mathrm{i}} .
\end{aligned}
$$


Its logarithm, $W[J, \bar{J}]=\log Z[J, \bar{J}]$ is the free energy and generates the connected correlation functions, which as pointed out before, are classified by boundary (possibly disconnected $D$-coloured) graphs.

\subsection{From Graph Calculus to Tensor Field Theory}

For a deeper exposition and motivation of the terminology and proofs of the results exposed in this section, we refer to [53].

Some objects of interest in tensor field theory are functionals generated by graphs (e.g. the free energy). By this we mean expansions in graphs with functions (or distributions) as coefficients. For a graph $g \in H \subset \mathrm{G}_{D}$ we recall that $2 k(g)$ denotes its number of vertices. One is interested in collections of functions

$$
\left\{u_{g}: \mathbb{Z}^{D \times k(g)} \rightarrow \mathbb{C}\right\}_{g \in H}
$$

and in their generating functionals

$$
U[J, \bar{J}]=\sum_{g \in H \subset G_{D}} u_{g} \star \mathbb{J}(g), \quad \text { where } u_{g} \star \mathbb{J}(g):=\sum_{\mathbf{X} \in \mathbb{Z}^{D \cdot k}}\left(u_{g}(\mathbf{X})\right) \mathbb{J}(g)(\mathbf{X}) .
$$

Here $\mathbb{J}(g)(\mathbf{X})=\prod_{\alpha=1}^{k(g)} J_{\mathbf{x}^{\alpha}} \bar{J}_{\mathbf{y}^{\alpha}}$, where $\left\{\mathbf{y}^{\alpha}\right\}_{\alpha}$ are determined by $g$ and $\mathbf{X}$ through

$$
g_{*}(\mathbf{X})=\left(\mathbf{y}^{1}, \ldots, \mathbf{y}^{k(g)}\right) .
$$

The induced map $g_{*}$ is defined as follows. The $D$-tuple $\mathbf{x}^{\alpha}$ (resp. the $\mathbf{y}^{\alpha}$ ) indexes white (resp. black) vertices in a graph. Then $g_{*}: M_{D \times k(g)}(\mathbb{Z}) \rightarrow M_{D \times k(g)}(\mathbb{Z})$ is given by $\mathbf{X}=\left(\mathbf{x}^{1}, \ldots, \mathbf{x}^{k(g)}\right) \mapsto g_{*}(\mathbf{X})=\left(\mathbf{y}^{1}, \ldots, \mathbf{y}^{k}\right)$, where $y_{c}^{\alpha}=x_{c}^{\nu}$ (for $\alpha=$ $1, \ldots, k)$ if and only in the graph $g$ there exists a $c$-coloured edge starting at $\mathbf{x}^{\alpha}$ and ending at $\mathbf{y}^{\nu}$. As before, $\mathbf{X}$ is called momentum, but also each one of these arguments $\mathbf{x}^{\alpha}$ is referred to as (entering) momentum of the white vertex $J_{\mathbf{x}^{\alpha}}$. Similarly $\mathbf{y}^{\nu}$ is the (outgoing) momentum at the black vertex $\bar{J}_{\mathbf{y}^{v}}$; the terminology relies on Fig. 2. Although an ordering of the vertices is assumed, notice that $\mathbb{J}(g)$ is independent of it.

Consider the canonical system of graph-group actions introduced in (2.22)

$$
\left\{u_{g}, M_{D \times k(g)}(\mathbb{Z}), \operatorname{Aut}_{\mathrm{c}}(g)\right\}_{g \in H} .
$$

A very important domain where the graph derivatives shall be defined is the subspace $\mathcal{F}_{D, k(g)}$ of $\mathbb{Z}^{D \cdot k(g)} \simeq M_{D \times k(g)}(\mathbb{Z})$ consisting of points outside all the coloured diagonals, i.e.

$$
\begin{array}{r}
\mathcal{F}_{D, k}:=\left\{\left(\mathbf{y}^{1}, \ldots, \mathbf{y}^{k}\right) \in M_{D \times k}(\mathbb{Z}) \mid \quad y_{c}^{\alpha} \neq y_{c}^{v} \text { for all } c=1, \ldots, D\right. \\
\text { and } \alpha, v=1, \ldots, k, \alpha \neq v\} .
\end{array}
$$

For a connected graph $g$, the elements of $\operatorname{Aut}_{\mathrm{c}}(g)$ are a lift $\hat{\sigma}$ of an element $\sigma$ of the symmetric group $\mathfrak{S}(k(g))$ (see discussion below (2.22), or [53] for details). Using (3.2) and the induced map $g_{*}$ to define the next derivative

$$
\frac{\partial U[J, \bar{J}]}{\partial g(\mathbf{X})}=\left.\prod_{\alpha=1}^{k(g)} \frac{\delta}{\delta J_{\mathbf{x}^{\alpha}}} \frac{\delta}{\delta \bar{J}_{\mathbf{y}^{\alpha}}} U[J, \bar{J}]\right|_{J=\bar{J}=0}, \quad \mathbf{X}=\left(\mathbf{x}^{1}, \ldots, \mathbf{x}^{k(g)}\right) \in \mathcal{F}_{D, k(g)},
$$



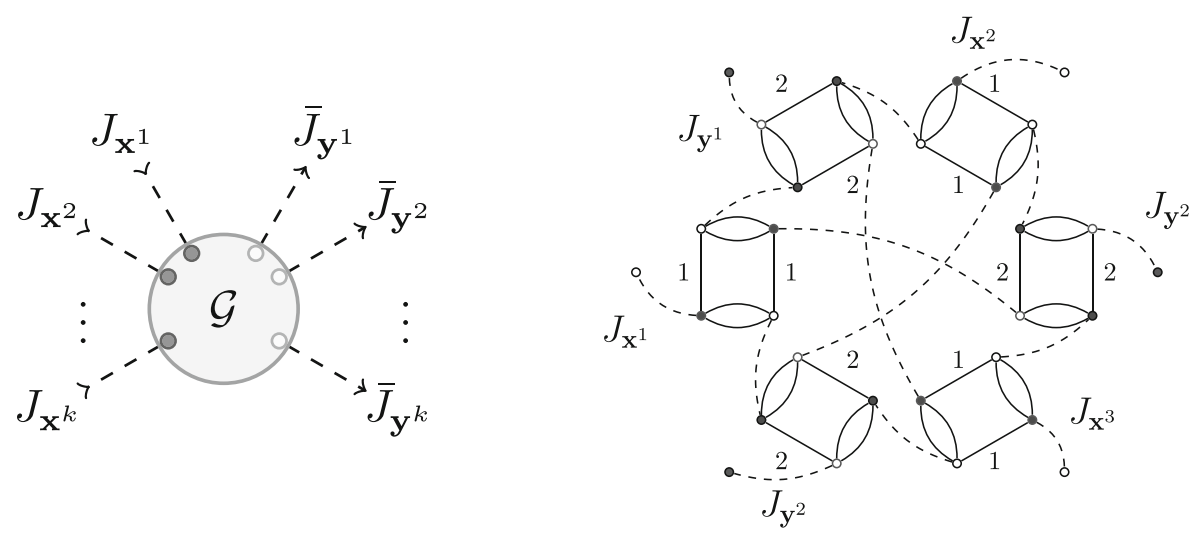

Fig. 2 Left. On the interpretation of the induced map $g_{*}$. One takes any representing graph $\mathcal{G}$ such that $\partial \mathcal{G}=g$. For the quartic melonic model (pillow-interactions) we can do so because it has been proven in [53] that the spectrum of boundary states is full, which is to say, the boundary graphs are all of $G_{D}$. Assuming an ordering on the white and on the black vertices, the components $\mathbf{y}^{\alpha}$ of $g_{*}\left(\mathbf{x}^{1}, \ldots, \mathbf{x}^{k}\right)=\left(\mathbf{y}^{1}, \ldots, \mathbf{y}^{k}\right)$ are determined by this picture, seeing the $\mathbf{x}^{\mu}$ 's as independent momenta entering the graph, with output $g_{*}\left(\mathbf{x}^{1}, \ldots, \mathbf{x}^{k}\right)$. Right. If $g=$, and we choose the numeration $x_{1}^{\alpha}=$ $y_{1}^{\alpha}$ for $\alpha=1,2,3$; there that $\mathcal{G}$ satisfying is shown. The map ()$_{*}\left(\mathbf{x}^{1}, \mathbf{x}^{2}, \mathbf{x}^{3}\right)=\left(\mathbf{y}^{1}, \mathbf{y}^{2}, \mathbf{y}^{3}\right)=$ $\left(\left(x_{1}^{1}, x_{2}^{2}, x_{3}^{3}\right)^{t},\left(x_{1}^{2}, x_{2}^{3}, x_{3}^{2}\right)^{t},\left(x_{1}^{3}, x_{2}^{1}, x_{3}^{2}\right)^{t}\right)$ is determined by following the $0 a$ momenta lines for each colour $a$

one can give this derivative the the meaning of (1.2) as a permutation of the arguments of $u_{g}$, that is

$$
\begin{aligned}
\frac{\partial U[J, \bar{J}]}{\partial g(\mathbf{X})} & =\sum_{\hat{\sigma} \in \operatorname{Aut}_{\mathrm{c}}(g)}\left(\sigma \cdot u_{g}\right)(\mathbf{X}), \text { where } \\
\left(\sigma \cdot u_{g}\right)\left(\mathbf{x}^{1}, \ldots, \mathbf{x}^{k(g)}\right): & =u_{g}\left(\mathbf{x}^{\sigma^{-1}(1)}, \ldots, \mathbf{x}^{\sigma^{-1}(k(g))}\right),
\end{aligned}
$$

for all $\mathbf{X}=\left(\mathbf{x}^{1}, \ldots, \mathbf{x}^{k(g)}\right) \in \mathcal{F}_{D, k(g)}$. The property ${ }^{11}$ Equation (3.4) is guaranteed by [53, Lem. 4] (see [54, Lem. $\left.4^{\prime}\right]$ too). An example of the graph derivative is

$$
\frac{\delta}{\delta{ }_{1} \mathfrak{g}^{1}(\mathbf{x}, \mathbf{y})}=\frac{\delta}{\delta J_{x_{1} y_{2} x_{3}}} \frac{\delta}{\delta \bar{J}_{x_{1} y_{2} y_{3}}} \frac{\delta}{\delta J_{y_{1} y_{2} y_{3}}} \frac{\delta}{\delta \bar{J}_{y_{1} x_{2} x_{3}}} \text { (no sum). }
$$

In order to obtain $\partial / \partial g(\mathbf{x}, \mathbf{y})$, with ${ }^{1}{ }^{1} 1$, , one has to evaluate the sources at zero. Beware $(\mathbf{x}, \mathbf{y}) \in \mathcal{F}_{3,2}$ for the graph derivative to enjoy of property (3.4).

Remark 1 Notice that in (3.4) it is summed over the group $\operatorname{Aut}_{\mathrm{c}}(g)$. We are therefore entitled to drop the inverse in the RHS in (3.5). However, if the sum is not over all the group, we will keep the right 'orientation' of the action, for the convention in single terms $\left(\sigma \cdot u_{g}\right)$ is important in that case.

\footnotetext{
${ }^{11}$ From the viewpoint of the graph-calculus, this is just a required condition. However, in order to realize the graph-calculus one has to prove that structures emerging in tensor field theory do satisfy the abstract properties of the graph-calculus.
} 
For graphs $h, g$, functions $u_{g}$ and functionals $U[J, \bar{J}]$ we abbreviate the usual notation as follows:

$$
U=\sum_{g} u_{g} g, \quad u_{g} g:=u_{g} \star \mathbb{J}(g) \quad \text { and } \quad g h:=\mathbb{J}(g) \mathbb{J}(h),
$$

and treat the latter as a product of graphs. The functional $U$ depends on the sources through the graphs according to (3.7). This product is not considered commutative, since the star $\star$ implies an ordering in the arguments of a function $u_{g h}$, which need not satisfy $u_{g h}=u_{h g}$. Now we exhibit the relation to the generating functional of group actions. With the product defined above, consider the functional $V \leq n$ that generates the connected correlation functions of TFT

$$
V^{\leq n}=\sum_{\mathcal{B} \in \mathrm{G}_{D}^{n}} G_{\mathcal{B}} \mathcal{B}
$$

(with $G_{\varnothing}=0$ ). Here $n$ is a large integer, and $\mathrm{G}_{D}^{n}$ is a finite set of coloured graphs whose elements $\mathcal{D}$ satisfy

$$
\#(\text { vertices of } \mathcal{D}) \leq 2 n
$$

In tensor field theory, the subindices of the functions corresponding to the graph $\mathcal{A} \amalg \mathcal{B}$ (also written as juxtaposition $\mathcal{A B}$ ) are rather denoted by $G_{\mathcal{A} \mid \mathcal{B}}$. These particular functions $G_{\mathcal{D}}$ satisfy invariance under $\operatorname{Aut}_{\mathrm{c}}(\mathcal{D})$; this means invariance under the Aut $_{\mathrm{c}}$-groups of the connected components of $\mathcal{D}$ and, for any isomorphic connected components $\mathcal{A}$ and $\mathcal{B}$ of $\mathcal{D}, G_{\ldots|\mathcal{A}| \ldots|\mathcal{B}| \ldots}=G_{\ldots|\mathcal{B}| \ldots|\mathcal{A}| \ldots}$.

The truncation (3.8) would declare vanishing all the floors above the $n$-th floor of the SDE-tower, but we can increase $n$ at desired accuracy. It bounds any graph $\mathcal{D}$ appearing in an non-identically vanishing correlator $G_{\mathcal{D}}$ to have $n$ components at most. In rank 3, since the canonical (optimal in number of vertices) 3-coloured graph of genus $g$ has $4 g+2$ vertices, this truncation bounds the genus through $2 g+1 \leq n$, making higher-genera boundary states vanish. We write the infinite sums keeping in mind that we mean their $n \rightarrow \infty$ limit. The coloured Borel transform of $V^{\infty}=$ $\lim _{n \rightarrow \infty} V^{\leq n}$ is called the free energy:

$$
W=\mathrm{B}_{\mathrm{c}}\left(V^{\infty}\right)=\sum_{\mathcal{D} \in \mathrm{G}_{D}} \frac{1}{\left|\operatorname{Aut}_{\mathrm{c}}(\mathcal{D})\right|} G_{\mathcal{D}} \mathcal{D}
$$

This equation holds in ' $N=1$-units' and this assumption is innocuous within the scope of this article. However, if one plans to proceed perturbatively in $1 / N$, the realistic case that drops this simplification ought to be addressed. Adding the power counting conjectured in [50] that scales $G_{\mathcal{D}} \rightarrow N^{\gamma(\mathcal{D})} G_{\mathcal{D}}$, where $\gamma(\mathcal{D})$ is certain factor already determined for the 2-pt and 4-pt functions, would help analysing the convergence of $W$ (see Section 7).

This free energy functional (but not only this) corresponds to a system of graphgroup actions that has the following constituents:

- $\quad$ For each graph $\mathcal{B}, \mathscr{V}(\mathcal{B})=M_{D \times k(\mathcal{B})}(\mathbb{Z})$. 
- For a disconnected graph $\mathcal{D}=\amalg_{p} \mathcal{B}_{p}$ one has

$$
\mathscr{N}(\mathcal{D})=M_{D \times k(\mathcal{D})}(\mathbb{Z})=\prod_{p} \mathscr{N}\left(\mathcal{B}_{p}\right) .
$$

- There is an action of each $\operatorname{Aut}_{\mathrm{c}}\left(\mathcal{B}_{p}\right)$ on $M_{D \times k\left(\mathcal{B}_{p}\right)}(\mathbb{Z})$. Since $\sigma \in \operatorname{Aut}_{\mathrm{c}}\left(\mathcal{B}_{p}\right) \subset$ $\mathfrak{S}\left(k\left(\mathcal{B}_{p}\right)\right)$, precomposition by a function by $\sigma$ permuting the columns of $M_{D \times k\left(\mathcal{B}_{p}\right)}(\mathbb{Z})$ gives this action.

For connected graphs $\mathcal{B}_{i}$ and $\mathcal{B}_{j}$, the following holds [53, Lemma 4]:

$$
\frac{\delta \mathcal{B}_{i}}{\delta \mathcal{B}_{j}}=\delta_{i j} \operatorname{Aut}_{\mathrm{c}}\left(\mathcal{B}_{i}\right)
$$

on the domain $\mathcal{F}_{D, k\left(\mathcal{B}_{j}\right)} \subset M_{D, k\left(\mathcal{B}_{j}\right)}$. For general disconnected graphs $\mathcal{D}=\mathcal{B}_{1}^{\alpha_{1}} \amalg$ $\cdots \amalg \mathcal{B}_{m}^{\alpha_{m}}$

$$
\frac{\delta \mathcal{D}}{\delta \mathcal{D}}=\operatorname{Aut}_{\mathrm{c}}(\mathcal{D})=\operatorname{Aut}_{\mathrm{c}}\left(\mathcal{B}_{1}\right) \imath \mathfrak{S}\left(\alpha_{1}\right) \times \cdots \times \operatorname{Aut}_{\mathrm{c}}\left(\mathcal{B}_{n}\right) \imath \mathfrak{S}\left(\alpha_{m}\right)
$$

on $\mathcal{F}_{D, k(\mathcal{D})} \subset M_{D \times k(\mathcal{D})}(\mathbb{Z})$. One can then operate with functionals, now without needing to evaluate the sources at 0 . That is,

$$
\frac{\delta U[J, \bar{J}]}{\delta g(\mathbf{X})}=\prod_{\alpha=1}^{k(g)} \frac{\delta}{\delta J_{\mathbf{X}^{\alpha}}} \frac{\delta}{\delta \bar{J}_{\mathbf{y}^{\alpha}}} U .
$$

This object is, unlike the function $\partial U / \partial g$, a functional that can be graph-derived again (without getting something a trivial result) and get again a functional generated by graphs. Contrast this with (3.3), whose result is a function.

Another important functional in the next derivation is the so-called $Y$-term that emerged in the derivation of the Ward-Takahashi identity [53] and which encodes all the pertinent insertions of $2 p$ point functions into $2 p-2$ point functions for all $p$.

The expression to order six is given in [51, Lemma 4.1], but this article only will evoke the $Y$-term up to order four, located in Appendix A. For this paper, it is sufficient to additionally know the expression

$$
Y_{x}^{(c)}=\sum_{\mathcal{B} \in \mathrm{G}_{D}} \mathfrak{f}_{\mathcal{B}, x}^{(c)} \mathcal{B}
$$

where $c \in\{1, \ldots, D\}$ is a colour and $x \in \mathbb{Z}$. It is important to notice that unlike $W$ (for which we set $G_{\varnothing}=0$ ), there is a non-vanishing constant term $\mathfrak{f}_{\varnothing, x}^{(c)}$ in $Y_{x}^{(c)}$. For $\mathcal{B}$ with more than 2 vertices, each function coefficient $\mathfrak{f}_{\mathcal{B}, x}^{(c)}$ of a graph $\mathcal{B}$ denotes a triple propagator contraction of vertices from with vertices of the graph $\mathcal{A}$ having $2 k(\mathcal{A})=2+2 k(\mathcal{B})$ vertices and such that the whole contraction's boundary is $\mathcal{B}$. An illustration is given in Fig. 3, but see [53] in order to understand the role of the number $x$ (here not used) as well as the definition of $Y$-term, if needed beyond the expressions given here in Appendix A. Although $G_{\mathcal{A}}$ is symmetric with respect to action of $\operatorname{Aut}_{\mathrm{c}}(\mathcal{A})$, the resulting insertion need not to be $\operatorname{Aut}_{\mathrm{c}}(\mathcal{B})$-symmetric.

\footnotetext{
${ }^{13}$ The cone of a graph is formed by doubling the number of its vertices, and attaching to each vertex a 0 coloured edge. The boundary of the cone of a graph is the original graph.
} 


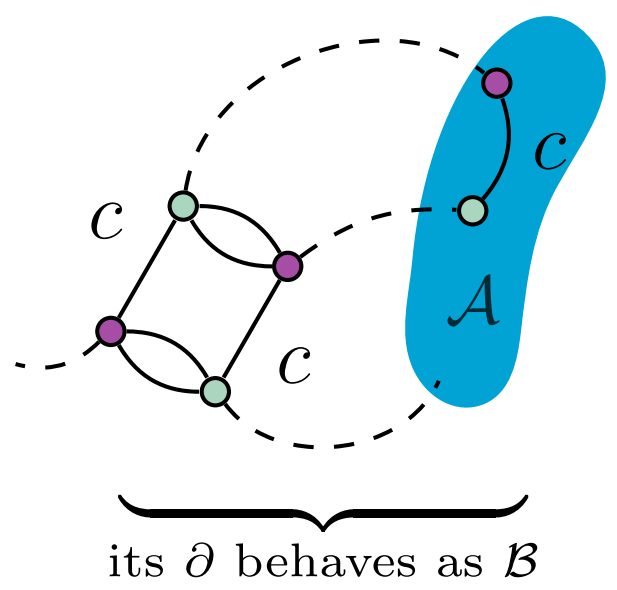

Fig. 3 For a fixed colour $c$, this picture interprets the $Y$-term for rank-3 theories, as a certain functional spanned by graphs, $Y^{(c)}=\sum_{\mathcal{B}} \mathfrak{f}_{\mathcal{B}}^{(c)} \mathcal{B}$. For graphs $\mathcal{A}$ with at least four vertices, the term $\mathfrak{f}_{\mathcal{B}}^{(c)}$ are constructed out of those correlation functions $G_{\mathcal{A}}$ having the next property: when $\mathcal{A}$ (or to be precise the cone $^{13}$ of $\mathcal{A}$ ) is triply contracted with a colour- $c$ quartic melonic ('pillow') vertex, as shown is this picture, the boundary of the resulting graph is $\mathcal{B}$. It is a condition that two of the vertices of the interaction pillow are contracted with two vertices directly joined by a $c$-coloured edge, but there might be more edges between those two vertices. This explains the appearance of $\mathfrak{f}_{\mathcal{B}}^{(c)}$ in the SDE's for $\mathcal{B}$, as will be proven. This description still does not fully determine $\mathfrak{f}_{\mathcal{B}}^{(c)}$, since $Y_{x}^{(c)}$ still depends on another parameter $x$ that determines how to contract the third leg, not shown here. For a rigorous definition we refer to [53]

In order to derive any of the $2 p$-point $\mathrm{SDE}^{14}$ we shall employ the graph calculus $\mathscr{C}(\mathfrak{h})$ with variables $\mathfrak{h}=$ connected, closed, $D$-coloured graphs with $\leq 2(p+1)$ vertices\}, being the system of graph-group actions the canonical one given by automorphism groups (see (2.22) above for details).

\section{Disconnected-Boundary Schwinger-Dyson Equations}

The next section introduces the model whose SDE are found in Section 4.2.

\subsection{The Quartic Melonic Tensor Field Theory}

The $\varphi_{D, \mathrm{~m}}^{4}$-theory is the model with quartic interaction vertices $V[\varphi, \bar{\varphi}]=$ $\lambda \sum_{a=1}^{D} \operatorname{Tr}_{V_{a}}(\varphi, \bar{\varphi})$. These vertices are sometimes called pillows, since the graphs they correspond to have that appearance:

$$
V_{a}=\underbrace{a}_{a} \bigoplus_{D} \quad a=1, \ldots, D .
$$

\footnotetext{
${ }^{14}$ Here we mean the melonic quartic model. The bound on the vertices is model dependent and justified in the statement of Theorem 1 .
} 
We analyse this theory with an abstract Laplacian $E: \mathbb{Z}^{D} \rightarrow \mathbb{R}_{\geq 0}$ as propagator, $S_{0}[\varphi, \bar{\varphi}]=\operatorname{Tr}_{\ominus}(\bar{\varphi}, E \varphi)=\sum_{\mathbf{x}} \bar{\varphi}_{\mathbf{x}} E_{\mathbf{x}} \varphi_{\mathbf{x}}$, assumed here to satisfy the following technical assumption: for each colour $c$, the difference

$$
E\left(t_{c}, s_{c}\right):=E_{p_{1} \ldots t_{c} \ldots p_{D}}-E_{p_{1} \ldots s_{c} \ldots p_{D}}
$$

is independent of the fixed momenta in colours different from $c$. Such kind of technical conditions permit to exploit the Ward-Identity and are common. In matrix field theory [32, Thm. 2.3], this is analogous to the assumed injectivity of $n \mapsto E_{n}$ for the generalised matrix Laplacian $E_{\text {matrix }}=\operatorname{diag}\left(E_{n}\right)_{n \in \mathbb{N}}$ there.

\subsection{Main Result}

We prepare ${ }^{15}$ now some notions and notations needed for the formulation of the main result. Let $\mathcal{R}$ be a connected graph, $2 r$ its number of vertices, and $X \in \mathcal{F}_{r, D}$. Given a colour $c$ and a numeration $w^{1}, \ldots, w^{r}$ of the black vertices of $\mathcal{R}$ we set, for any $1 \leq \rho \leq r$

$$
\operatorname{Br}^{(2)}(\mathcal{R}, \rho, c):=\left\{\tau \in\{1, \ldots, r\} \mid \varsigma_{c}\left(\mathcal{R}, w^{\rho}, w^{\tau}\right) \text { is disconnected }\right\} .
$$

Thus, $\tau$ belonging to this set indexes the vertices $w^{\tau}$ at which a swap of the colour $c$ edge at $w^{\tau}$ and $w^{\rho}$ disconnects $\mathcal{R}$. In other words, $\operatorname{Br}^{(2)}(\mathcal{R}, \rho, c)$ indexes $c$ coloured edges of $\mathcal{R}$ that, paired with the $c$-coloured edge at $w^{\rho}$, form a 2-bridge. If $\operatorname{Br}^{(2)}(\mathcal{R}, \rho, c) \neq \emptyset$, then $\mathcal{R}$ must be, in particular, 3-edge connected.

Example 6 The coloured utility graph satisfies $\mathrm{Br}^{(2)}(\bowtie, \rho, c)=\emptyset$ for any colour $c$ and vertex $\rho$ ('no edge-swap separates it'). On the other hand, if $\{\rho, \mu\}=\{1,2\}$ label the two black vertices of the pillow ${ }^{191}$, then

$$
\mathrm{Br}^{(2)}\left(1 g_{1}, \rho, c=1\right)=\{\mu\} \text { and } \mathrm{Br}^{(2)}(1 / g 1, \rho, c)=\emptyset \text { for } c=2,3
$$

Notation of the theorem Let $\mathcal{D}$ be a $D$-coloured graph with $2 d$ vertices. By [53, Thm. 1] $\mathcal{D}$ is a boundary graph of the $\varphi_{D, \mathrm{~m}}^{4}$-theory and $G_{\mathcal{D}} \not \equiv 0$. Given any $\mathbf{X} \in$ $\mathcal{F}_{d, D}$, we select an outgoing momentum $\mathbf{s}=\mathbf{y}^{\beta}$ listed in

$$
\left(\mathbf{y}^{1}, \ldots, \mathbf{y}^{\beta}, \ldots, \mathbf{y}^{d}\right)=\mathcal{D}_{*}(\mathbf{X}) \in \mathcal{F}_{d, D}
$$

This $\mathbf{s}$ determines both a connected graph component $\mathcal{R}$ of $\mathcal{D}$, and an $r$-tuple $X_{0} \in \mathcal{F}_{r, D}$ of momenta, being $2 r$ the number of vertices in $\mathcal{R}$, by asking that $\mathbf{s}$ appears listed in the $r$-tuple $\mathcal{R}_{*}\left(X_{0}\right)$, particularly. Different choices of the distinguished momentum variable $\mathbf{s}-$ say $\mathbf{s}=\mathbf{y}^{\beta_{1}}$ and $\mathbf{s}=\mathbf{y}^{\beta_{2}}$ - lead to a different SDE whenever the respective $\beta_{1}$-th and $\beta_{2}$-th black vertices lie on different connected components; or, less obviously, when such vertices do lie in the same connected graph, $\mathcal{R}$, but they are not related by a non-trivial element of $\operatorname{Aut}_{\mathrm{c}}(\mathcal{R})$. In particular, if

\footnotetext{
${ }^{15}$ We come back to the usual notation for graphs or 'bubbles'.
} 
the distinguished connected component $\mathcal{R}$ has no symmetries, then there are exactly half as many SDE's as vertices of $\mathcal{R}$, to wit $k(\mathcal{R})$ equations (for that connected component).

For the rest of components of $\mathcal{D}$ we write $\mathcal{Q}(\mathcal{D}=\mathcal{R} \amalg \mathcal{Q})$. We factorise $\mathcal{Q}$ in $\alpha_{u}$ copies of pairwise non-isomorphic connected graphs $\left\{Q_{u}\right\}_{u=1, \ldots, n}$ :

$$
\mathcal{Q}=Q_{1}^{\alpha_{1}} \amalg \ldots \amalg Q_{n}^{\alpha_{n}}, \quad\left(Q_{i}^{\alpha_{i}}=\amalg_{A=1}^{\alpha_{i}} Q_{i}\right)
$$

We split the $d$-tuple $\mathbf{X}$ into momenta $X_{0}$ of $\mathcal{R}$ and momenta $\mathbb{X}$ of $\mathcal{Q}$, so that $\left(X_{0}, \mathbb{X}\right)=$ $\mathbf{X}$, up to reordering. For $\tau \in \mathrm{Br}^{(2)}(\mathcal{R}, \beta, c)$ we can therefore write

$$
\varsigma_{c}(\mathcal{R} ; \beta, \tau)=\mathcal{R}^{\prime} \amalg \mathcal{R}^{\prime \prime},
$$

and accordingly split the momentum $X_{0}$ in momenta $X_{0}^{\prime}$ of $\mathcal{R}^{\prime}$ and $X_{0}^{\prime \prime}$ of $\mathcal{R}^{\prime \prime}, X_{0}=$ $\left(X_{0}^{\prime}, X_{0}^{\prime \prime}\right)$. Furthermore, for any factorising pair of graphs

$$
\mathcal{C}, \mathcal{B} \in \mathrm{G}_{D} \text { with } \mathcal{C} \amalg \mathcal{B} \stackrel{\mathrm{c}}{\sim} \mathcal{Q}
$$

we define the two functions $H_{\mathcal{C}, \mathcal{B}}^{(c, \tau)}$ and $I_{\mathcal{C}, \mathcal{B}}^{(c)}$ by the following products:

$$
\begin{aligned}
H_{\mathcal{C}, \mathcal{B}}^{(c, \tau)} & =\Theta_{\mathcal{Q}}\left\{G_{\mathcal{R}^{\prime} \mid \mathcal{C}}\left(X_{0}^{\prime} ; \bullet\right) \times G_{\mathcal{R}^{\prime \prime} \mid \mathcal{B}}\left(X_{0}^{\prime \prime} ; \bullet\right)\right\}, \quad\left(\tau \in \operatorname{Br}^{(2)}(\mathcal{R}, \beta, c)\right) \\
I_{\mathcal{C}, \mathcal{B}}^{(c)} & =\frac{1}{\left|\operatorname{Aut}_{\mathrm{c}}(\mathcal{B})\right|} \Theta_{\mathcal{Q}}\left\{\mathfrak{f}_{\mathcal{C}, s_{c}}^{(c)} \times G_{\mathcal{R} \mid \mathcal{B}}\left(X_{0} ; \bullet\right)\right\}
\end{aligned}
$$

where the reorderings $\Theta_{\mathcal{Q}}$ refer only to the graph components of the graphs in the pair $(\mathcal{C}, \mathcal{B})$. The pivotal term, appearing in each equation, is

$$
\mathfrak{f}_{\varnothing, s_{c}}^{(c)}=Y_{s_{c}}^{(c)}[0,0]=\sum_{\mathbf{q}_{\hat{c}}} G_{\bigodot}^{(2)}\left(s_{c}, \mathbf{q}_{\hat{c}}\right),
$$

where the $G_{\ominus}^{(2)}$ denotes the 2-point function and $\left(s_{c}, \mathbf{q}_{\hat{c}}\right)$ is shorthand notation for the $D$-tuple $\left(q_{1}, \ldots, q_{c-1}, s_{c}, q_{c+1}, \ldots, q_{D}\right)$. Notice the implicit colour-reordering bringing the $s_{c}$ argument to the $c$-th place. The sum in last equation then runs over the $D-1$ arguments of $\mathbf{q}_{\hat{c}}=\left(q_{1}, \ldots, q_{c-1}, q_{c+1}, \ldots, q_{D}\right)$.

The appearance of the 2-point function $G_{2}^{(2)}$ is due to the fact that the coefficient of a certain graph $\mathcal{B}$ in the $Y$-term is related to correlation functions indexed by graphs that after certain dipole removal yield $\mathcal{B}$ (however, this description does not fully determine $Y$ - for a true definition, see [53, pages 619-624]). Since the melon is a 
$D$-dipole, removing the dipole yields the empty graph. For other graphs, the coefficients are given in the Appendix A.

Theorem 1 For the $\varphi_{D, \mathrm{~m}}^{4}$-theory with kinetic term (4.2), the Schwinger-Dyson equations for the disconnected graph $\mathcal{D}$ read, for the particular vertex choice $\mathbf{s}=\mathbf{y}^{\beta}$, as follows:

$$
\begin{aligned}
& G_{\mathcal{D}}^{(2 k)}(\mathbf{X}) \\
& =\frac{(-2 \lambda)}{E_{\mathbf{s}}} \sum_{c=1}^{D}\left\{\sum_{\hat{\sigma} \in \operatorname{Aut}_{c}(\mathcal{D})} \sigma \cdot \mathfrak{f}_{\mathcal{D}, s_{c}}^{(c)}(\mathbf{X})\right. \\
& +\sum_{\rho \neq \beta} \frac{1}{E\left(y_{c}^{\rho}, s_{c}\right)}\left[\frac{\partial W[J, \bar{J}]}{\partial \varsigma_{c}(\mathcal{D} ; \beta, \rho)}(\mathbf{X})-\frac{\partial W[J, \bar{J}]}{\partial \varsigma_{c}(\mathcal{D} ; \beta, \rho)}\left(\left.\mathbf{X}\right|_{\left.s_{c} \rightarrow y_{c}^{\rho}\right)}\right]\right. \\
& -\sum_{b_{c}} \frac{1}{E\left(s_{c}, b_{c}\right)}\left[G_{\mathcal{D}}^{(2 k)}(\mathbf{X})-G_{\mathcal{D}}^{(2 k)}\left(\left.\mathbf{X}\right|_{s_{c} \rightarrow b_{c}}\right)\right] \\
& +\sum_{\tau \in \mathrm{Br}^{(2)}(\mathcal{R} ; \beta, c)}\left[\sum_{\substack{(\mathcal{C}, \mathcal{B}) \in \mathrm{G}_{D} \cup \mathrm{G}_{D} \\
(\mathcal{C} \cup \mathcal{B}) \sim \mathcal{Q}}} \sum_{\Omega \in \operatorname{Aut}_{c}(\mathcal{Q})} \frac{\left(\Omega \cdot H_{\mathcal{B}, \mathcal{C}}^{(c, \tau)}\right)(\mathbb{X})-\left(\Omega \cdot H_{\mathcal{B}, \mathcal{C}}^{(c, \tau)}\right)\left(\left.\mathbb{X}\right|_{\left.s_{c} \rightarrow y_{c}^{\tau}\right)}\right.}{E\left(y_{c}^{\tau}, s_{\mathcal{C}}\right)}\right] \\
& \left.+\sum_{\substack{(\mathcal{C}, \mathcal{B}) \in \mathrm{G}_{D} \amalg \mathrm{G}_{D} \\
(\mathcal{C} \sqcup \mathcal{B}) \sim \mathcal{Q}}} \sum_{\Omega \in \operatorname{Aut}_{\mathrm{c}}(\mathcal{Q})}\left(\Omega \cdot I_{\mathcal{B}, \mathcal{C}}^{(c)}\right)(\mathbb{X})\right\}
\end{aligned}
$$

Proof By definition,

$$
G_{\mathcal{D}}=\frac{\partial W}{\partial \mathcal{D}}=\left.\left[\frac{\delta}{\delta \mathcal{Q}}\left(\frac{\delta W}{\delta \mathcal{R}}\right)\right]\right|_{J=\bar{J}=0}
$$

Spelled out, this means that

$$
G_{\mathcal{D}}(\mathbf{X})=\left.\left\{\left[\prod_{i=1}^{n} \frac{\delta^{\alpha_{i}}}{\delta \mathcal{Q}_{1}^{i} \delta \mathcal{Q}_{2}^{i} \cdots \delta \mathcal{Q}_{\alpha_{i}}^{i}}(\mathbb{X})\left(\prod_{\alpha=1}^{r} \frac{\delta}{\delta J_{\mathbf{x}_{0}^{\alpha}}} \frac{\delta}{\delta \bar{J}_{\mathbf{y}_{0}^{\alpha}}}\right)\right] W[J, \bar{J}]\right\}\right|_{J=\bar{J}=0}
$$

To compute $G_{\mathcal{D}}$, one needs to start then with a functional derivative of $W$ with respect to a source, which we choose to be $\bar{J}_{\mathbf{s}}=\bar{J}_{\mathbf{y}^{\beta}}$. The partition function $Z[J, \bar{J}]=\exp (W[J, \bar{J}])$ has been shown [53] to satisfy

$$
\frac{\delta W}{\delta \bar{J}_{\mathbf{s}}}=\frac{1}{E_{\mathbf{s}}}\left\{J_{\mathbf{s}}-\left.\mathrm{e}^{-W}\left(\frac{\partial V(\varphi, \bar{\varphi})}{\partial \bar{\varphi}_{\mathbf{s}}}\right)\right|_{\substack{\varphi \rightarrow \delta / \delta \bar{J} \\ \bar{\varphi} \rightarrow \delta / \delta J}} \mathrm{e}^{+W}\right\}
$$


The colour-c-WTI [53, Thm. 2] can used in the first line to directly compute:

$$
\begin{aligned}
&\left.\left(\frac{\partial V(\varphi, \bar{\varphi})}{\partial \bar{\varphi}_{\mathbf{s}}}\right)\right|_{\substack{\varphi \rightarrow \delta / \delta \bar{J} \\
\bar{\varphi} \rightarrow \delta / \delta J}}=2 \lambda \sum_{c}\left\{\sum _ { b _ { c } } \frac { \delta } { \delta \overline { J } _ { \mathbf { s } _ { \hat { c } } b _ { c } } } \left(\delta_{s_{c} b_{c}} Y_{s_{c}}^{(c)}[J, \bar{J}] \cdot\right.\right. \\
&\left.\left.+\sum_{\mathbf{b}_{\hat{c}}} \frac{1}{E\left(b_{c}, s_{c}\right)}\left(\bar{J}_{\mathbf{b}} \frac{\delta}{\delta \bar{J}_{\mathbf{b}_{\hat{c}} s_{c}}}-J_{\mathbf{b}_{\hat{c}} s_{c}} \frac{\delta}{\delta J_{\mathbf{b}}}\right)\right) Z[J, \bar{J}]\right\} \\
&=2 \lambda \sum_{c}\left\{\frac{\delta Y_{s_{c}}^{(c)}[J, \bar{J}]}{\delta \bar{J}_{\mathbf{s}}} \cdot Z[J, \bar{J}]+Y_{s_{c}}^{(c)}[J, \bar{J}] \cdot \frac{\delta Z[J, \bar{J}]}{\delta \bar{J}_{\mathbf{s}}}\right. \\
&\left.+\sum_{\mathbf{b}} \frac{1}{E\left(b_{c}, s_{c}\right)} \frac{\delta}{\delta \bar{J}_{\mathbf{s}_{\hat{c}}} b_{c}}\left(\bar{J}_{\mathbf{b}} \frac{\delta}{\delta \bar{J}_{\mathbf{b}_{\hat{c}} s_{c}}}-J_{\mathbf{b}_{\hat{c}} s_{c}} \frac{\delta}{\delta J \mathbf{b}}\right) Z[J, \bar{J}]\right\} \\
&=2 \lambda \sum_{c}\left\{\frac{\delta Y_{s_{c}}^{(c)}[J, \bar{J}]}{\delta \bar{J}_{\mathbf{s}}} \cdot Z[J, \bar{J}]+Y_{s_{c}}^{(c)}[J, \bar{J}] \cdot \frac{\delta Z[J, \bar{J}]}{\delta \bar{J}_{\mathbf{s}}}\right. \\
&+\sum_{b_{c}} \frac{1}{E\left(b_{c}, s_{c}\right)} \frac{\delta Z[J, \bar{J}]}{\delta \bar{J}_{\mathbf{s}}}+\sum_{\mathbf{b}} \frac{\bar{J}_{\mathbf{b}}}{E\left(b_{c}, s_{c}\right)} \frac{\delta \bar{J}_{\mathbf{s}_{\hat{c}} b_{c}} \delta[J, \bar{J}]}{\overline{\mathbf{b}}_{\hat{c}} s_{c}} \\
&\left.-\sum_{\mathbf{b}} \frac{1}{E\left(b_{c}, s_{c}\right)} J_{\mathbf{b}_{\hat{c}} s_{c}} \frac{\delta^{2} Z[J, \bar{J}]}{\delta \bar{J}_{\hat{s}_{\hat{c}} b_{c}} \delta J_{\mathbf{b}}}\right\} \\
&=2 \lambda \sum_{c}\left(A_{c}(\mathbf{s})-B_{c}(\mathbf{s})+C_{c}(\mathbf{s})+D_{c}(\mathbf{s})+F_{c}(\mathbf{s})\right), \quad(4.7)
\end{aligned}
$$

where $\mathbf{s}_{\hat{c}} b_{c}=\left(s_{1} \ldots, s_{a-1}, b_{c}, s_{c+1} \ldots, s_{D}\right)$ and $\mathbf{b}_{\hat{c}} s_{c}=\left(b_{1} \ldots, b_{c-1}, s_{c}, b_{c+1} \ldots\right.$, $b_{D}$ ), and each of the summands is given by

$$
\begin{aligned}
A_{c}(\mathbf{s}) & =Y_{s_{c}}^{(c)}[J, \bar{J}] \cdot \frac{\delta Z[J, \bar{J}]}{\delta \bar{J}_{\mathbf{s}}}, \\
B_{c}(\mathbf{s}) & =\sum_{\mathbf{b}} \frac{J_{\mathbf{b}_{\hat{c}} s_{c}}}{E\left(b_{c}, s_{c}\right)} \frac{\delta^{2} Z[J, \bar{J}]}{\delta \bar{J}_{\hat{s}_{\hat{c}} b_{c}} \delta J_{\mathbf{b}}}, \\
C_{c}(\mathbf{s}) & =\sum_{b_{c}} \frac{1}{E\left(b_{c}, s_{c}\right)} \frac{\delta Z[J, \bar{J}]}{\delta \bar{J}_{\mathbf{s}}}, \\
D_{c}(\mathbf{s}) & =\sum_{\mathbf{b}} \frac{\bar{J}_{\mathbf{b}}}{E\left(b_{c}, s_{c}\right)} \frac{\delta^{2} Z[J, \bar{J}]}{\delta \bar{J}_{\hat{s}_{c} b_{c}} \delta \bar{J}_{\mathbf{b}_{\hat{c}} s_{c}}}, \\
F_{c}(\mathbf{s}) & =\frac{\delta Y_{s_{c}}^{(c)}[J, \bar{J}]}{\delta \bar{J}_{\mathbf{s}}} \cdot Z[J, \bar{J}],
\end{aligned}
$$


for any $c=1, \ldots, D$. In this new derivation, it is convenient to work with

$$
\begin{aligned}
\mathrm{e}^{-W} A_{c}(\mathbf{s}) & =Y_{s_{c}}^{(c)}[J, \bar{J}] \cdot \frac{\delta W[J, \bar{J}]}{\delta \bar{J}_{\mathbf{s}}}, \\
\mathrm{e}^{-W} B_{c}(\mathbf{s}) & =\sum_{\mathbf{b}} \frac{J_{\mathbf{b}_{\hat{c}} s_{c}}}{E\left(b_{c}, s_{c}\right)}\left[\frac{\delta^{2} W[J, \bar{J}]}{\delta \bar{J}_{\mathbf{s}_{\hat{c}} b_{c}} \delta J_{\mathbf{b}}}+\frac{\delta W[J, \bar{J}]}{\delta J_{\mathbf{b}}} \frac{\delta W[J, \bar{J}]}{\left.\delta \bar{J}_{\mathbf{s}_{\hat{c}} b_{c}}\right]}\right. \\
\mathrm{e}^{-W} C_{c}(\mathbf{s}) & =\sum_{b_{c}} \frac{1}{E\left(b_{c}, s_{c}\right)} \frac{\delta W[J, \bar{J}]}{\delta \bar{J}_{\mathbf{s}}}, \\
\mathrm{e}^{-W} D_{c}(\mathbf{s}) & =\sum_{\mathbf{b}} \frac{\bar{J}_{\mathbf{b}}}{E\left(b_{c}, s_{c}\right)}\left[\frac{\delta^{2} W[J, \bar{J}]}{\delta \bar{J}_{\mathbf{s}_{\hat{c}} b_{c}} \delta \bar{J}_{\mathbf{b}_{\hat{c}} s_{c}}}+\frac{\delta W[J, \bar{J}]}{\delta \bar{J}_{\mathbf{s}_{\hat{c}} b_{c}}} \frac{\delta W[J, \bar{J}]}{\delta \bar{J}_{\mathbf{b}_{\hat{c}} s_{c}}}\right], \\
\mathrm{e}^{-W} F_{c}(\mathbf{s}) & =\frac{\delta Y_{s_{c}}^{(c)}[J, \bar{J}]}{\delta \bar{J}_{\mathbf{s}}} .
\end{aligned}
$$

Notice the presence of the product of derivatives in the $B_{c}$ and $D_{c}$ terms. These are called $B_{c}^{\text {(prod) }}$ and $D_{c}^{\text {(prod) }}$, respectively. The other two terms (which already appeared on the SDE's for connected boundaries) containing a double derivative are denoted by $B_{c}^{(\mathrm{dd})}$ and $D_{c}^{(\mathrm{dd})}$, respectively.

Next, we use the freedom to numerate momenta starting with the component $\mathcal{R}$,

$$
X_{0}=\left(\mathbf{x}_{0}^{1}, \ldots, \mathbf{x}_{0}^{r}\right) \in \mathcal{F}_{D, r}, \mathcal{R}_{*}\left(X_{0}\right)=\left(\mathbf{y}_{0}^{1}, \ldots, \mathbf{y}_{0}^{r}\right)
$$

and $\mathbf{s}=\mathbf{y}^{\beta}$. For each item in the list

$$
(\mathfrak{m}, M) \in\{(\mathfrak{a}, A),(\mathfrak{b}, B),(\mathfrak{c}, C),(\mathfrak{d}, D),(\mathfrak{f}, F)\},
$$

we define the following functions:

$$
\mathfrak{m}_{c}(\mathbf{X} ; \mathbf{s} ; \mathcal{D}):=\left.\frac{\delta^{\alpha}}{\delta \mathcal{Q}^{\alpha}(\mathbb{X})} \prod_{\substack{\alpha \neq \beta \\ 0 \leq \alpha \leq r \\ \nu=1, \ldots, r}} \frac{\delta}{\delta \bar{J}_{\mathbf{y}_{0}^{\alpha}}} \frac{\delta}{\delta J_{\mathbf{x}_{0}^{v}}}\left[\mathrm{e}^{-W[J, \bar{J}]} M_{c}(\mathbf{s})\right]\right|_{J=\bar{J}=0}
$$

The splitting of $\mathfrak{b}$ and $\mathfrak{d}$ into terms $\mathfrak{b}^{(\mathrm{dd})}, \mathfrak{b}^{\text {(prod) }}, \mathfrak{d}^{(\mathrm{dd})}, \mathfrak{d}^{\text {(prod) }}$, respectively, still makes sense. We now determine all coefficients, beginning with the easiest.

The $\mathfrak{c}_{c}$ and $\mathfrak{f}_{c}$ terms are readily computed:

$$
\begin{aligned}
& \mathfrak{c}_{c}(\mathbf{X} ; \mathbf{s} ; \mathcal{D})=\sum_{b_{c}} \frac{1}{E\left(b_{c}, s_{c}\right)} G_{\mathcal{D}}^{(2 k)}(\mathbf{X}), \\
& \mathfrak{f}_{c}(\mathbf{X} ; \mathbf{s} ; \mathcal{D})=\sum_{\hat{\pi} \in \operatorname{Aut}_{\mathcal{c}}(\mathcal{D})} \pi^{*} \mathfrak{f}_{\mathcal{D}}^{(c)}(\mathbf{X}) .
\end{aligned}
$$

The term $\mathfrak{c}_{c}$ itself is not finite, but a term arising from one of the three functions will render it finite. The three remaining $\mathfrak{m}_{c}$-functions involve derivatives of products of 
functionals. We first observe that the $2 r-1$ derivatives in the sources complete the graph derivative $\delta W / \delta \mathcal{R}$, so the $\mathfrak{a}_{c}$ yields

$$
\begin{aligned}
\mathfrak{a}_{c}(\mathbf{X} ; \mathbf{s} ; \mathcal{D}) & =\left.\frac{\delta^{\alpha}}{\delta \mathcal{Q}^{\alpha}(\mathbb{X})} \prod_{\substack{\alpha \neq \beta \\
0 \leq \alpha \leq r \\
\nu=1, \ldots, r}} \frac{\delta}{\delta \bar{J}_{\mathbf{y}_{0}^{\alpha}}} \frac{\delta}{\delta J_{\mathbf{x}_{0}^{v}}}\left(Y_{s_{c}}^{(c)}[J, \bar{J}] \cdot \frac{\delta W[J, \bar{J}]}{\delta \bar{J}_{\mathbf{s}}}\right)\right|_{J=\bar{J}=0} \\
& =\left.\frac{\delta^{\alpha}}{\delta \mathcal{Q}^{\alpha}(\mathbb{X})}\left(Y_{s_{c}}^{(c)}[J, \bar{J}] \cdot \frac{\delta W[J, \bar{J}]}{\delta \mathcal{R}\left(X_{0}\right)}\right)\right|_{J=\bar{J}=0}
\end{aligned}
$$

Notice that, after evaluation in the sources at 0 ,

$$
\frac{\partial}{\partial \mathcal{A}} \cdots \frac{\partial}{\partial \mathcal{A}^{\prime}} \frac{\partial}{\partial \mathcal{R}\left(X_{0}\right)} W[J, \bar{J}]=G_{\mathcal{R}|\mathcal{A}| \cdots \mid \mathcal{A}^{\prime}}\left(X_{0}, \bullet\right)
$$

holds for any boundary graphs $\mathcal{A}, \ldots, \mathcal{A}^{\prime}$. Using the formula for the graph derivative of products (Lemma 3 ) and subsequently Lemma 2 one deduces

$$
\begin{aligned}
& \mathfrak{a}_{c}(\mathbf{X} ; \mathbf{s} ; \mathcal{D})=\sum_{\substack{\mathcal{B}, \mathcal{C} \\
\mathcal{B} \cup \mathcal{C} \sim \mathcal{Q}}} \sum_{\substack{\mathrm{c}}} \sum_{\operatorname{Aut}_{\mathrm{c}}(\mathcal{Q})} \\
& \frac{1}{\left|\operatorname{Aut}_{\mathrm{c}}(\mathcal{R} \amalg \mathcal{B})\right|} \Omega \cdot \Theta_{\mathcal{Q}}\left\{G_{\mathcal{R} \mid \mathcal{B}}\left(\sigma\left(X_{0}\right) ; \bullet\right) \times \mathfrak{f}_{\mathcal{C}, s_{c}}^{(c)}\right\} \\
& =\sum_{\substack{\mathcal{B}, \mathcal{C} \\
\mathcal{B} \amalg \mathcal{C} \sim \mathcal{Q}}} \sum_{\Omega \in \operatorname{Aut}_{\mathrm{c}}(\mathcal{Q})} \frac{1}{\left|\operatorname{Aut}_{\mathrm{c}}(\mathcal{B})\right|} \Omega \cdot \Theta_{\mathcal{Q}}\left\{G_{\mathcal{R} \mid \mathcal{B}}\left(X_{0} ; \bullet\right) \times \mathfrak{f}_{\mathcal{C}, s_{c}}^{(c)}\right\} \\
& =\sum_{\substack{\mathcal{B}, \mathcal{C} \\
\mathcal{B} \cup \mathcal{C} \sim \mathcal{Q}}} \sum_{\Omega \in \operatorname{Aut}_{\mathrm{c}}(\mathcal{Q})} \Omega \cdot I_{\mathcal{C}, \mathcal{B}}^{(c)}(\mathbb{X}) .
\end{aligned}
$$

We compute now ${ }^{16}$ the term $\mathfrak{d}_{c}$. We can split $D_{c}$ into the double derivative contribution $D_{c}^{(\mathrm{dd})}$ and the product of single derivatives $D_{c}^{(\text {prod) }}$. If we decompose $\delta^{\alpha} / \delta \mathcal{Q}^{\alpha}$

\footnotetext{
${ }^{16}$ The part of the calculation of the contributions of the double derivatives $\delta^{2} W / \delta J \delta \bar{J}$ or $\delta^{2} W / \delta \bar{J} \delta \bar{J}$ to $\mathfrak{b}_{c}$ and $\mathfrak{d}_{c}$ is shortened, due to the very similar derivation provided already in [51].
} 
and the rest of derivatives implied in $\mathcal{R}$ into single functional derivatives,

$$
\begin{aligned}
\mathcal{O}(\bar{J})+ & \prod_{\substack{\alpha \neq \beta \\
\nu=1, \ldots, d}} \frac{\delta}{\delta \bar{J}_{\mathbf{y}^{\alpha}}} \frac{\delta}{\delta J_{\mathbf{x}^{v}}}\left(\mathrm{e}^{-W} D_{c}^{(\mathrm{dd})}(\mathbf{s})\right) \\
= & \prod_{\substack{\alpha \neq \beta ; v\\
}} \frac{\delta}{\delta \bar{J}_{\mathbf{y}^{\alpha}}} \frac{\delta}{\delta J_{\mathbf{x}^{v}}}\left[\sum_{\mathbf{b}} \frac{1}{E\left(b_{c}, s_{c}\right)} \bar{J}_{\mathbf{b}} \frac{\delta^{2} W[J, \bar{J}]}{\delta \bar{J}_{\mathbf{s}_{\hat{c}} b_{c}} \delta \bar{J}_{\mathbf{b}_{\hat{c}} s_{c}}}\right] \\
= & \sum_{\substack{\rho=1 \\
\rho \neq \beta}}^{d} \prod_{\substack{\beta \neq \alpha \neq \rho \\
\nu=1, \ldots, d}} \frac{\delta}{\delta \bar{J}_{\mathbf{y}^{\alpha}}} \frac{\delta}{\delta J_{\mathbf{x}^{v}}}\left[\sum_{\mathbf{b}} \frac{\delta_{\mathbf{y}^{\rho}}^{\mathbf{b}}}{E\left(b_{c}, s_{c}\right)} \frac{\delta^{2} W[J, \bar{J}]}{\delta \bar{J}_{\mathbf{s}_{\hat{c}} b_{c}} \delta \bar{J}_{\mathbf{b}_{\hat{c}} s_{c}}}\right] \\
= & \sum_{\substack{\rho=1 \\
\rho \neq \beta}}^{\substack{\alpha ;(\beta \neq \alpha \neq \rho) \\
\nu=1, \ldots, k}} \frac{\delta}{\delta \bar{J}_{\mathbf{y}^{\alpha}}} \frac{\delta}{\delta J_{\mathbf{x}^{v}}}\left[\frac{1}{E\left(y_{c}^{\rho}, s_{c}\right)} \frac{\delta^{2} W[J, \bar{J}]}{\delta \bar{J}_{\mathbf{s}_{\hat{c}} y_{c}} \delta \bar{J}_{\mathbf{y}_{\hat{c}}^{\rho} s_{c}}}\right] .
\end{aligned}
$$

This is, after evaluation at $\bar{J}=J=0$, all the (coloured) graphs obtained from $\mathcal{D}$ (also implying the other connected components) after the colour- $c$ swapping at $\mathbf{s}=\mathbf{y}^{\beta}$ and $\mathbf{y}^{\rho}$ : for $\rho \neq \beta$, i.e. running over black vertices skipping $\bar{J}_{\mathbf{s}}=\bar{J}_{\mathbf{y}} \beta$. Thus the derivatives on $D_{c}^{(\mathrm{dd})}$ contribute

$$
\sum_{\substack{\rho \neq \beta \\ \rho, \text { any black vertex }}} \frac{1}{E\left(y_{c}^{\rho}, s_{c}\right)} \frac{\partial W[J, J]}{\partial \varsigma_{c}(\mathcal{D} ; \beta, \rho)(\mathbf{X})} \text { for } \rho \neq \beta .
$$

To fully compute $D_{c}$, we add now the product of derivatives, $D_{c}^{(\text {prod) }}$. Unlike $D_{c}^{(\mathrm{dd})}$, with $D_{c}^{\text {(prod) }}$ one can first see the effect of applying the rest of the derivatives of the graph $\mathcal{R}$, that is those with momenta $\left(\mathbf{x}_{0}^{\alpha}\right)_{\alpha}$ and $\left(\mathbf{y}_{0}^{\alpha}\right)_{\alpha \neq \beta}$. This is due to the product of derivatives of $W$, which forces both factors to be derived with respect to momenta in the same graph in order not to vanish. Thus,

$$
\begin{aligned}
& \prod_{\substack{\alpha \neq \beta \\
\nu=1, \ldots, r}} \frac{\delta}{\delta \bar{J}_{\mathbf{y}_{0}^{\alpha}}} \frac{\delta}{\delta J_{\mathbf{x}_{0}^{\nu}}}\left(\mathrm{e}^{-W} D_{c}^{(\operatorname{prod})}(\mathbf{s})\right)+\mathcal{O}(\bar{J}) \\
& =\prod_{\alpha \neq \beta ; \nu} \frac{\delta}{\delta \bar{J}_{\mathbf{y}_{0}^{\alpha}}} \frac{\delta}{\delta J_{\mathbf{x}_{0}^{v}}}\left[\sum_{\mathbf{b}} \frac{\bar{J}_{\mathbf{b}}}{E\left(b_{c}, s_{c}\right)} \frac{\delta W}{\delta \bar{J}_{\mathbf{s}_{\hat{c}} b_{c}}} \frac{\delta W}{\delta \bar{J}_{\mathbf{b}_{\hat{c}} s_{c}}}\right] \\
& =\sum_{\rho \neq \beta}^{r} \prod_{\substack{\alpha \neq \beta(\alpha \neq \rho) \\
\nu=1, \ldots, r}} \frac{\delta}{\delta \bar{J}_{\mathbf{y}_{0}^{\alpha}}} \frac{\delta}{\delta J_{\mathbf{x}_{0}^{v}}^{v}}\left[\sum_{\mathbf{b}} \frac{\delta_{\mathbf{y}^{\rho}}^{\mathbf{b}}}{E\left(b_{c}, s_{c}\right)} \frac{\delta W}{\delta \bar{J}_{\mathbf{s}_{\hat{c}} b_{c}}} \frac{\delta W}{\delta \bar{J}_{\mathbf{b}_{\hat{c}} s_{c}}}\right] \\
& =\sum_{\rho \neq \beta}^{r} \frac{1}{E\left(y_{c}^{\rho}, s_{c}\right)} \prod_{\substack{\alpha \neq \beta(\alpha \neq \rho) \\
\nu=1, \ldots, r}} \frac{\delta}{\delta \bar{J}_{\mathbf{y}_{0}^{\alpha}}} \frac{\delta}{\delta J_{\mathbf{x}_{0}^{v}}}\left[\frac{\delta W}{\delta \bar{J}_{\mathbf{y}_{\hat{c}}^{\beta} y_{c}^{\rho}}} \frac{\delta W}{\delta \bar{J}_{\mathbf{y}_{\hat{c}}^{\rho} y_{c}^{\beta}}}\right],
\end{aligned}
$$

where in the last step we only used that $\mathbf{s}=\mathbf{y}^{\beta}=\mathbf{y}_{0}^{\beta}$. It is evident that these two derivatives in the square bracket form of a colour- $c$ edge swap at $\mathbf{y}_{0}^{\beta}$ and $\mathbf{y}_{0}^{\rho}$, but in 
order not to lead to a vanishing term, they also have to lie on a different component of a graph (as otherwise a graph derivative would be incomplete). It is therefore additionally required that the graph is disconnected after the swapping at $\mathbf{y}_{0}^{\beta}$ and $\mathbf{y}_{0}^{\rho}$, that is that there are connected graphs $\mathcal{R}_{1}$ and $\mathcal{R}_{2}$, such that

$$
\varsigma_{c}(\mathcal{R} ; \beta, \rho)=\mathcal{R}_{1} \amalg \mathcal{R}_{2} \quad \text { if and only if } \quad \rho \in \operatorname{Br}(\mathcal{R}, \beta, c) .
$$

Therefore

$$
\begin{aligned}
& \frac{\delta^{\alpha}}{\delta \mathcal{Q}^{\alpha}(\mathbb{X})} \prod_{\substack{\alpha \neq \beta \\
\nu=1, \ldots, r}} \frac{\delta}{\delta \bar{J}_{\mathbf{y}_{0}^{\alpha}}} \frac{\delta}{\delta J_{\mathbf{x}_{0}^{v}}}\left(\mathrm{e}^{-W} D_{c}^{(\operatorname{prod})}(\mathbf{s})\right)+\mathcal{O}(\bar{J}) \\
= & \sum_{\tau \in \operatorname{Br}(\mathcal{R}, \beta, c)} \frac{1}{E\left(y_{c}^{\tau}, s_{c}\right)} \frac{\delta^{\alpha}}{\delta \mathcal{Q}^{\alpha}(\mathbb{X})} \prod_{\substack{\alpha \neq \beta(\alpha \neq \rho) \\
\nu=1, \ldots, r}} \frac{\delta}{\delta \bar{J}_{\mathbf{y}_{0}^{\alpha}}} \frac{\delta}{\delta J_{\mathbf{x}_{0}^{v}}}\left[\frac{\delta W}{\delta \bar{J}_{y_{\hat{c}}^{\beta} y_{c}^{\tau}}} \frac{\delta W}{\delta \bar{J}_{\mathbf{y}_{\hat{c}}^{\tau} y_{c}^{\beta}}}\right] . \\
= & \sum_{\tau \in \operatorname{Br}(\mathcal{R}, \beta, c)} \frac{1}{E\left(y_{c}^{\tau}, s_{c}\right)} \frac{\delta^{\alpha}}{\delta \mathcal{Q}^{\alpha}(\mathbb{X})}\left(\frac{\delta W}{\delta \mathcal{R}_{1}\left(X_{0}^{\prime}\right)} \frac{\delta W}{\delta \mathcal{R}_{2}\left(X_{0}^{\prime \prime}\right)}\right) .
\end{aligned}
$$

At this place we use the multivariable graph calculus Leibniz rule (Lemma 3), namely

$$
\begin{aligned}
& \left.\frac{\delta^{\alpha}}{\delta \mathcal{Q}^{\alpha}(\mathbb{X})} \prod_{\substack{\alpha \neq \beta \\
\nu=1, \ldots, r}} \frac{\delta}{\delta \bar{J}_{\mathbf{y}_{0}^{\alpha}}} \frac{\delta}{\delta J_{\mathbf{x}_{0}^{v}}}\left(\mathrm{e}^{-W} D_{c}^{(\text {prod })}(\mathbf{s})\right)\right|_{J=\bar{J}=0} \\
= & \sum_{\tau \in \operatorname{Br}(\mathcal{R}, \beta, c)} \frac{1}{E\left(y_{c}^{\tau}, s_{c}\right)} \sum_{\substack{\mathcal{B}, \mathcal{C} \\
\mathcal{B} \mathcal{C}_{\mathrm{c}}^{\sim} \mathcal{Q}^{\alpha}}} \sum_{\Omega \in \operatorname{Aut}_{\mathrm{c}}\left(\mathcal{Q}^{\alpha}\right)} \\
= & \sum_{\tau \in \operatorname{Br}(\mathcal{R}, \beta, c)} \frac{1}{E\left(y_{c}^{\tau}, s_{c}\right)} \sum_{\substack{\mathcal{B}, \mathcal{C} \\
\mathcal{B} \mathcal{C}_{\sim}^{\sim} \mathcal{Q}^{\alpha}}} \sum_{\Omega \in \operatorname{Aut}_{\mathrm{c}}\left(\mathcal{Q}^{\alpha}\right)}\left(\Omega \cdot H_{\mathcal{B}, \mathcal{C}}^{(c, \tau)}\right)(\mathbb{X}) .
\end{aligned}
$$

In summary, the $\mathfrak{d}_{c}$-term is given by

$$
\begin{aligned}
\mathfrak{d}_{c}(\mathbf{X} ; \mathbf{s} ; \mathcal{D}) & =\sum_{\substack{\rho \neq \beta \\
\rho, \text { any black vertex }}} \frac{1}{E\left(y_{c}^{\rho}, s_{c}\right)} \frac{\partial W[J, J]}{\partial \varsigma_{c}(\mathcal{D} ; 1, \rho)}(\mathbf{X}) \\
& +\sum_{\tau \in \operatorname{Br}(\mathcal{R}, \beta, c)} \frac{1}{E\left(y_{c}^{\tau}, s_{c}\right)} \sum_{\substack{\mathcal{B}, \mathcal{C} \\
\mathcal{B} \mathcal{C}_{\mathcal{c}}^{\mathcal{Q}^{\alpha}}}} \sum_{\Omega \in \operatorname{Aut}_{c}\left(\mathcal{Q}^{\alpha}\right)}\left(\Omega \cdot H_{\mathcal{B}, \mathcal{C}}\right)(\mathbb{X}) .(4.1
\end{aligned}
$$

As for the derivatives on $B_{c}(\mathbf{s})$, we divide the derivation in two parts. One concerns the double derivative, $B_{c}^{(\mathrm{dd})}$ :

$$
\mathcal{O}(J)+\prod_{\substack{\alpha \neq \beta \\ \nu=1, \ldots, d}} \frac{\delta}{\delta \bar{J}_{\mathbf{y}^{\alpha}}} \frac{\delta}{\delta J_{\mathbf{x}^{\nu}}} B_{c}^{(\mathrm{dd})}(\mathbf{s})
$$




$$
\begin{aligned}
& =\prod_{\alpha \neq \beta ; \nu} \frac{\delta}{\delta \bar{J}_{\mathbf{y}^{\alpha}}} \frac{\delta}{\delta J_{\mathbf{x}^{v}}}\left[\sum_{\mathbf{b}} \frac{1}{E\left(b_{c}, s_{c}\right)} J_{\mathbf{b}_{\hat{c}} s_{c}} \frac{\delta^{2} W[J, \bar{J}]}{\delta \bar{J}}{ }_{\mathbf{s}_{\hat{c}} b_{c}} \delta J_{\mathbf{b}}\right] \\
& =\sum_{\theta=1}^{d} \prod_{\alpha \neq \beta ; \nu \neq \theta} \frac{\delta}{\delta \bar{J}_{\mathbf{y}^{\alpha}}} \frac{\delta}{\delta J_{\mathbf{x}^{v}}}\left[\sum_{b_{c}} \frac{1}{E\left(b_{c}, s_{c}\right)} \delta_{x_{c}^{\theta}}^{s_{c}} \frac{\delta^{2} W[J, \bar{J}]}{\delta \bar{J}_{\mathbf{s}_{\hat{c}} b_{c}} \delta J_{\mathbf{x}_{\hat{c}}^{\beta} b_{c}}}\right] \\
& =\prod_{\alpha \neq \beta ; v \neq \gamma} \frac{\delta}{\delta \bar{J}_{\mathbf{y}^{\alpha}}} \frac{\delta}{\delta J_{\mathbf{x}^{v}}}\left[\sum_{b_{c}} \frac{1}{E\left(b_{c}, x_{c}^{\gamma}\right)} \frac{\delta^{2} W[J, \bar{J}]}{\delta \bar{J}_{\mathbf{s}_{\hat{c}} b_{c}} \delta J_{\mathbf{x}_{\hat{c}}^{\gamma} b_{c}}}\right] .
\end{aligned}
$$

since there is a single vertex $\mathbf{x}^{\gamma}, \gamma=\gamma(c)$, with $x_{c}^{\gamma}=s_{c}$, so $\delta_{x_{c}^{\theta}}^{s_{c}}=\delta_{x_{c}^{\theta}}^{s_{c}} \delta_{\theta}^{\gamma}$. The term $\delta^{2} W[J, \bar{J}] / \delta \bar{J}_{\mathbf{s}_{\hat{c}} b_{c}} \delta J_{\mathbf{x}_{\hat{c}}^{\gamma} b_{c}}$, is selected by $\delta_{x_{c}^{\beta}}^{s_{c}}$ leads to $\partial Z /\left.\partial \mathcal{D}(\mathbf{X})\right|_{x_{c}^{\gamma} \rightarrow b_{c}}$, after taking all the rest of derivatives, with the single coordinate $x_{c}^{\gamma}$ being substituted by (the running) $b_{c}$. But when

$$
b_{c} \in\left\{y_{c}^{1}, y_{c}^{2}, \ldots, \widehat{y_{c}^{\beta}}, \ldots, y_{c}^{d}\right\},
$$

one does not have exactly a 'graph derivative', since we are evaluating it not in $\mathcal{F}_{D, d}$, but in one of its diagonals of colour $c$. A direct computation yields then a second contribution to $\mathfrak{b}_{c}^{(\mathrm{dd})}$ (the third and fourth lines below):

$$
\begin{aligned}
& \mathfrak{b}_{c}^{(\mathrm{dd})}(\mathbf{X} ; \mathbf{s} ; \mathcal{D}) \\
= & \sum_{b_{c}} \frac{1}{E\left(b_{c}, x_{c}^{\gamma}\right)} \\
\times & G_{\mathcal{D}}^{(2 k)}\left(\mathbf{x}^{1}, \ldots, \mathbf{x}^{\gamma-1},\left(x_{1}^{\gamma}, \ldots, x_{a-1}^{\gamma}, b_{c}, x_{a+1}^{\gamma}, \ldots x_{D}^{\gamma}\right), \mathbf{x}^{\gamma+1}, \ldots, \mathbf{x}^{d}\right) \\
+ & \sum_{\rho>1} \frac{1}{E\left(x_{c}^{\kappa(\rho)}, x_{c}^{\gamma}\right)} \\
\times & \frac{\partial W[J, \bar{J}]}{\partial \zeta_{c}(\mathcal{D} ; 1, \rho)}\left(\mathbf{x}^{1}, \ldots,\left(x_{1}^{\gamma}, \ldots, x_{a-1}^{\gamma}, x_{c}^{\kappa(\rho)}, x_{a+1}^{\gamma}, \ldots x_{D}^{\gamma}\right), \ldots, \mathbf{x}^{d}\right) .(4 .)
\end{aligned}
$$

where $\kappa(\rho)$ is defined in Fig. 4 (i.e. $x_{c}^{\kappa(\rho)}=y_{c}^{\rho}$ ).

The last computation is $\mathfrak{b}_{c}^{\text {(prod) }}$,

$$
\begin{aligned}
& \mathfrak{b}_{c}^{(\operatorname{prod})}(\mathbf{X} ; \mathbf{s} ; \mathcal{D}) \\
& =\left.\frac{\delta^{\alpha}}{\delta \mathcal{Q}^{\alpha}(\mathbb{X})} \prod_{\substack{\alpha \neq \beta \\
0 \leq \alpha \leq r \\
\nu=1, \ldots, r}} \frac{\delta}{\delta \bar{J}_{\mathbf{y}_{0}^{\alpha}}} \frac{\delta}{\delta J_{\mathbf{x}_{0}^{v}}}\left[\sum_{\mathbf{b}} \frac{J_{\mathbf{b}_{\hat{c}} s_{c}}}{E\left(b_{c}, s_{c}\right)} \frac{\delta W[J, \bar{J}]}{\delta J_{\mathbf{b}}} \frac{\delta W[J, \bar{J}]}{\delta \bar{J}_{\mathbf{s}_{\hat{c}} b_{c}}}\right]\right|_{J=\bar{J}=0} .
\end{aligned}
$$

This computation is quite similar to the one for the $\mathfrak{d}_{c}^{\text {(prod) }}$-term, presented above, with the only difference that the evaluation is not at $\mathbb{X}$, but at $\left.\mathbb{X}\right|_{s_{c} \rightarrow y_{c}^{\tau}}$. The less trivial part in that derivation is to figure out, which non-zero contributions come from

$$
\frac{\delta W[J, \bar{J}]}{\delta J_{b_{c} \mathbf{x}_{c}^{\gamma}}} \frac{\delta W[J, \bar{J}]}{\delta \bar{J}_{\mathbf{s}_{\hat{c}} b_{c}}} .
$$



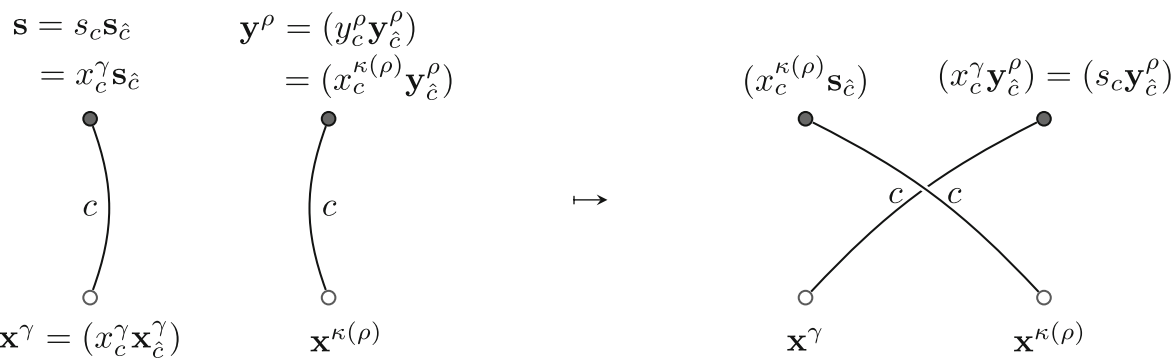

Fig. 4 The swapping operation $\varsigma_{c}$; variables are relevant in the proof

Because of the repetition of $b_{c}$ in both factors, it seems that the term vanishes after deriving it. However, $b_{c}$ runs, and it does so also through the particular $c$-coloured entries of the black vertices of $\mathcal{R}$,

$$
b_{c} \in\left\{y_{0, c}^{1}, y_{0, c}^{2}, \ldots, \widehat{y_{0, c}^{\beta}}, \ldots, y_{0, c}^{d}\right\} .
$$

Only if we also require that $b_{c} \in\left\{y_{c}^{\tau} \mid \tau \in \operatorname{Br}(\mathcal{R}, \beta, c)\right\}$, we guarantee that each one of those factors forms a graph derivative. However, notice that momentum in the white vertex $\mathbf{x}^{\gamma}=\left(s_{c} \mathbf{x}_{\hat{c}}^{\gamma}\right)$ has changed to $\left(y_{c}^{\tau} \mathbf{x}_{\hat{c}}^{\gamma}\right)$. Thus, one changes $X_{0}$ into $\left.X_{0}\right|_{s_{c} \rightarrow y_{c}^{\tau}}$. Therefore

$$
\mathfrak{b}_{c}^{(\operatorname{prod})}(\mathbf{X} ; \mathbf{s} ; \mathcal{D})=\left.\sum_{\tau \in \operatorname{Br}(\mathcal{R}, \beta, c)} \frac{1}{E\left(y_{c}^{\tau}, s_{c}\right)} \frac{\delta^{\alpha}}{\delta \mathcal{Q}^{\alpha}(\mathbb{X})} \frac{\delta W}{\delta \mathcal{R}_{1}\left(X_{0}^{\prime}\right)} \frac{\delta W}{\delta \mathcal{R}_{2}\left(X_{0}^{\prime \prime}\right)}\right|_{\substack{J=\bar{J}=0 \\ s_{c} \rightarrow y_{c}^{\tau}}} .
$$

We apply again Lemma 3 and find that

$$
\mathfrak{b}_{c}^{\text {(prod) }}(\mathbf{X} ; \mathbf{s} ; \mathcal{D})=\sum_{\tau \in \operatorname{Br}^{(2)}(\mathcal{R} ; \beta, c)} \sum_{\substack{\mathcal{C}, \mathcal{B}) \in G_{D} \amalg G_{D} \\(\mathcal{C} \cup \mathcal{B}) \sim \mathcal{Q}}} \sum_{\Omega \in \operatorname{Aut}_{\mathrm{c}}(\mathcal{Q})} \frac{\left.\left(\Omega \cdot H_{\mathcal{B}, \mathcal{C}}\right)(\mathbb{X})\right|_{s_{c} \rightarrow y_{c}^{\tau}}}{E\left(y_{c}^{\tau}, s_{C}\right)}
$$

Due to (4.7), one has

$$
\begin{aligned}
\frac{\partial W[J, \bar{J}]}{\partial \mathcal{D}(\mathbf{X})} & =\prod_{i} \frac{\delta^{\alpha_{i}}}{\delta \mathcal{Q}_{i}\left(X_{1}^{i}\right) \cdots \mathcal{Q}_{i}\left(X_{\alpha_{i}}^{i}\right)}\left\{\prod _ { \substack { \alpha \neq \beta \\
\nu = 1 , \ldots , k } } \frac { \delta } { \delta \overline { J } _ { \mathbf { y } ^ { \alpha } } } \frac { \delta } { \delta J _ { \mathbf { x } ^ { v } } } \left(\left(-2 \lambda E_{\mathbf{s}}^{-1}\right)\right.\right. \\
& \left.\left.\times \sum_{c=1}^{D} \mathrm{e}^{-W}\left[A_{c}(\mathbf{s})+C_{c}(\mathbf{s})+D_{c}(\mathbf{s})+F_{c}(\mathbf{s})-B_{c}(\mathbf{s})\right]\right)\right\}\left.\right|_{\substack{J=0 \\
J=0}} \\
& =\frac{(-2 \lambda)}{E_{\mathbf{s}}} \sum_{c}\left(\mathfrak{a}_{c}(\mathbf{X} ; \mathbf{s} ; \mathcal{D})+\mathfrak{c}_{c}(\mathbf{X} ; \mathbf{s} ; \mathcal{D})+\mathfrak{d}_{c}^{(\mathrm{dd})}(\mathbf{X} ; \mathbf{s} ; \mathcal{D})\right. \\
& +\mathfrak{d}_{c}^{(\operatorname{prod})}(\mathbf{X} ; \mathbf{s} ; \mathcal{D})+\mathfrak{f}_{c}(\mathbf{X} ; \mathbf{s} ; \mathcal{D}) \\
& \left.-\mathfrak{b}_{c}^{(\mathrm{dd})}(\mathbf{X} ; \mathbf{s} ; \mathcal{D})-\mathfrak{b}_{c}^{(\operatorname{prod})}(\mathbf{X} ; \mathbf{s} ; \mathcal{D})\right)
\end{aligned}
$$

This is precisely $G_{\mathcal{D}}(\mathbf{X})$ and the result follows. 


\section{Towards Higher-Dimensional Tutte Equations}

We compare the result with matrix models loop equations and, their equivalent, Tutte equations that count discrete surfaces.

\subsection{Tutte Equations and Matrix Models}

This material is based on the exposition by Eynard [26, Ch. $1 \& 2$ ]. There, three facts are proven:

1. The generating functions $\mathcal{T}_{l_{1}+1, l_{2}, \ldots, l_{\kappa}}$ of connected maps ${ }^{17}$ with $\kappa$ marked faces (boundaries) of perimeters $l_{1}+1, \ldots, l_{\kappa}$ satisfy Tutte equations:

$$
\mathcal{T}_{l+1}^{(0)}=\sum_{j=3}^{d} \lambda_{j} \mathcal{T}_{l-j-1}^{(0)}+\sum_{l_{1}+l_{2}=l-1} \mathcal{T}_{l_{1}}^{(0)} \mathcal{T}_{l_{2}}^{(0)}
$$

in the planar, single-boundary $(\kappa=1)$ case, while for all $g \geq 1$, setting $K=$ $\left\{l_{2}, \ldots, l_{\kappa}\right\}$

$$
\begin{aligned}
\mathcal{T}_{l_{1}+1, K}^{(g)} & =\sum_{\alpha=3}^{d} \lambda_{\alpha} \mathcal{T}_{l_{1}+\alpha-1, K}^{(g)}+\sum_{m=2}^{\kappa} l_{m} \mathcal{T}_{l_{1}+l_{m}-1, K \backslash\left\{l_{m}\right\}}^{(g)} \\
& +\sum_{j=0}^{l_{1}-1}\left[\mathcal{T}_{j, l_{1}-1-j, K}^{(g-1)}+\sum_{\substack{g_{1}+g_{2}=g \\
J \subset K}} \mathcal{T}_{j, J}^{\left(g_{1}\right)} \times \mathcal{T}_{l_{1}-1-j, K \backslash J}^{\left(g_{2}\right)}\right] .
\end{aligned}
$$

In the last two equations the superindex $h$ in $\mathcal{T}^{(h)}$ means the restriction to genus$h$ maps. Also the formal variables $\lambda_{\alpha}$ count the number $n_{\alpha}$ of $\alpha$-agons in each map. That is, the $t^{v(\mathfrak{m})} \lambda_{3}^{n_{3}(\mathfrak{m})} \cdots \lambda_{d}^{n_{d}(\mathfrak{m})}$-coefficient of $\mathcal{T}_{l_{1}, \ldots, l_{\kappa}}^{(h)}$ counts, modulo automorphisms, how many genus- $h$ maps $\mathfrak{m}$ are there, having precisely $n_{\alpha}(\mathfrak{m})$ $\alpha$-agons with marked faces of lengths $l_{m}>0(\alpha=3, \ldots, d ; m=1, \ldots, \kappa)$. To render this number finite, the variable $t$ counts the number of vertices $v(\mathfrak{m})$ of the map $\mathfrak{m}$. These $\mathcal{T}$-generating functions are not independent but related via Tutte equations - and in fact obey a rather universal relation known as Topological Recursion.

2. The matrix model

$$
Z=\int_{\text {formal }} \mathrm{d} M \mathrm{e}^{-\frac{N}{t}\left[\frac{M^{2}}{2}-V(M)\right]}, \quad V(x)=\sum_{\alpha=3}^{d} \lambda_{\alpha} \frac{x^{\alpha}}{\alpha},
$$

\footnotetext{
${ }^{17} \mathrm{~A}$ map is a concept slightly more general than a gluing of a collection of $n_{\alpha} \alpha$-gons by their sides $(\alpha \geq 3)$. Maps might have certain number $\kappa$ of marked faces of perimeters $l_{1}, \ldots, l_{\kappa} \geq 0$, Their Euler characteristic is $\chi=$ \#vertices - \#edges + \#unmarked faces $=2-2 g-\kappa$, being $g$ the genus of the map. The precise concept will not be needed here and we refer to [26, Section 1.1.2] for the definition in terms of permutations.
} 
satisfies Migdal's loop equations [44]

$$
\begin{aligned}
& \sum_{j=0}^{l_{1}-1}\left\langle\operatorname{Tr}\left(M^{j}\right) \operatorname{Tr}\left(M^{l_{1}-1-j}\right) \prod_{i=2}^{\kappa} \operatorname{Tr} M^{l_{i}}\right\rangle+\sum_{m=2}^{\kappa} l_{m}\left\langle\operatorname{Tr}\left(M^{l_{1}-1+l_{m}} \prod_{\substack{i=2 \\
i \neq m}}^{\kappa} \operatorname{Tr}\left(M^{l_{i}}\right)\right\rangle\right. \\
= & \frac{N}{t}\left\langle\operatorname{Tr}\left(M^{l_{1}} \cdot\left[M-V^{\prime}(M)\right]\right) \times \operatorname{Tr}\left(M^{l_{2}}\right) \cdots \operatorname{Tr}\left(M^{l_{\kappa}}\right)\right\rangle
\end{aligned}
$$

These expressions are the SDE for the matrix-valued correlator

$$
\left\langle M^{l_{1}} \operatorname{Tr}\left(M^{l_{2}}\right) \cdots \operatorname{Tr}\left(M^{l_{\kappa}}\right)\right\rangle,
$$

defined for a function $\Phi: M_{N}(\mathbb{R}) \rightarrow M_{N}(\mathbb{R})$ by

$$
\langle\Phi(M)\rangle=\frac{\int \mathrm{d} M \Phi(M) \mathrm{e}^{-\frac{N}{t} \operatorname{Tr}\left(M^{2} / 2-V(M)\right)}}{\int \mathrm{d} M \mathrm{e}^{-\frac{N}{t} \operatorname{Tr}\left(M^{2} / 2-V(M)\right)}} .
$$

Notice that these expressions imply the non-connected expectation values. The connected ones $\langle\cdots\rangle_{\text {connected }}$ can be obtained by tools from the probability theory relating the cumulants with the momenta (via incomplete Bell polynomials).

3. The crux of the matter is that Tutte Equations (5.1) hold if and only if the SDE (5.3) for the matrix model (5.2) do. The bridge is the following. For closed maps $\log Z$, the logarithm of the formal integral (5.2), is well-known to yield the generating function of connected closed maps (cf. [12]). The formal variables $\lambda_{\alpha}$ in both cases coincide: if only maps consisting of, say, triangulations and quadrangulations are to be counted, one sets a cubic and quartic interaction in the matrix model (in (5.2) $\lambda_{\alpha}=\delta_{3}^{\alpha} \lambda_{3}+\delta_{\alpha}^{4} \lambda_{4}$ ). For maps with $\kappa$ marked faces, if $x_{1}, \ldots, x_{\kappa}$ are formal variables and one defines $W_{\kappa}$ by

$$
W_{\kappa}\left(x_{1}, \ldots, x_{\kappa}\right)=\left\langle\operatorname{Tr} \frac{1}{x_{1}-M} \cdots \operatorname{Tr} \frac{1}{x_{\kappa}-M}\right\rangle_{\text {connected }},
$$

in the sense of Neumann series, then $\mathcal{T}_{l_{1} \ldots l_{K}}$ can be recovered by taking residues at $x_{i} \rightarrow \infty$ as follows:

$$
\mathcal{T}_{l_{1} \ldots l_{\kappa}}=(-1)^{\kappa} \operatorname{Res}_{x_{1} \rightarrow \infty} \ldots \operatorname{Res}_{x_{\kappa} \rightarrow \infty}\left[x_{1}^{l_{1}} \ldots x_{\kappa}^{l_{\kappa}} W_{\kappa}\left(x_{1}, \ldots, x_{\kappa}\right)\right] .
$$

Tutte equations (5.1) for all genera $g \in \mathbb{Z}_{\geq 0}$ emerge by taking the small- $t$ expansion $^{18} \mathcal{T}_{l_{1}+1, \ldots l_{\kappa}}=\sum_{g}(N / t)^{2-2 g-\kappa} \mathcal{T}_{l_{1}+1, \ldots l_{\kappa}}^{(g)}$.

\subsection{Parallel Between Tutte Equations and Disconnected- $\partial$ SDE of TFT}

We contrast now elements appearing in the SDE of Theorem 1 with Tutte equations, as well as the derivation of both sets.

The first parallel, depicted in Table 3, concerns the role of the boundaries in each framework.

\footnotetext{
${ }^{18}$ To see the subtleties between small- $t$ expansion and an $1 / N$-expansion we refer to [26, Section 1.2 .4$]$
} 
Table 1 Operations on the boundaries (marked face) for the connected boundary Tutte for random maps / Schwinger-Dyson equations for the matrix model $Z=\int \mathrm{d} M \mathrm{e}^{-\frac{N}{t}\left(\frac{M^{2}}{2}-V(M)\right)}$ with $V(x)=\sum_{\alpha=3}^{d} \lambda_{\alpha} x^{\alpha} / \alpha$

Single boundary Tutte / Schwinger Dyson Equations

\begin{tabular}{llll}
\hline Input & Outputs & Interpretation \\
\hline$l+1$ & $\rightarrow$ & $\{l-1+\alpha\}_{\alpha \geq 3}$ if $\left[x^{\alpha}\right] V(x) \neq 0$ & increase by $\alpha$ length of boundary \\
(boundary perimeter) & $\searrow \quad\{j, l-1-j\}_{j=0, \ldots, l-1}$ & splitting in 2 lengths, adding up to $l-1$
\end{tabular}

Moreover, we can regard each Tutte equation as a set of operations in the input, namely the perimeters $l_{1}+1, \ldots, l_{\kappa}$ of the marked faces, and distinguish the two cases $\kappa=1$ and $\kappa>1$. The output for the connected- $\partial$ Tutte equations (5.1a) is illustrated in Table 1; similarly, for the disconnected- $\partial$ Tutte equations Table 2 depicts the types of operations on the list of perimeters.

A second similitude is the role played by a distinguished boundary in each case. Tutte equations can be derived [26, Ch. 1] by distinguishing a single boundary length, say $l_{1}+1$, among the list of perimeters $l_{1}+1, \ldots, l_{\kappa}$, and seeing the possible effects ${ }^{19}$ that the removal of a marked edge of this $\left(l_{1}+1\right)$-agon has at the level of the $\mathcal{T}$ generating functions. In our TFT SDE, the role of that marked face is taken by the boundary component denoted $\mathcal{R}$. This undergoes the transformations explained in Table 4.

This new operations are an improvement of description given in [51]. Put into the Tutte Equations perspective, the analogue of Table 1 are precisely those operations on a connected $\partial$-graph of [51]. The contribution of Theorem 1 is to give the full set of operations described and interpreted in Table 4.

One can compare term by term Tutte vs. Schwinger-Dyson equations. The terminology refers to Tables 2 and 4 . They do not match $1: 1$, due to the fact that in TFT the number of operations on boundaries increases.

Finally, boundaries - and therefore SDE — of TFT are more complex than their matrix counterparts due to their lack of symmetry. As pointed out in Section 4.2, the

\footnotetext{
${ }^{19}$ Incidentally, this amounts to four possible scenarios that account for each one of the terms in (5.1b):

I. if the marked edge separates an ordinary face from a marked face: this accounts for the term $\lambda_{\alpha} \mathcal{T}_{l_{1}+\alpha-1, K}^{(g)}$

II. if the marked edge separates two marked marked faces $\rightarrow l_{m} \mathcal{T}_{l_{1}+l_{m}-1, K \backslash\left\{l_{m}\right\}}^{(g)}$

III. if the marked edge bounds twice the same face:
}

III.a and the marked edge is not a bridge $\rightarrow \mathcal{T}_{j, J}^{\left(g_{1}\right)} \times \mathcal{T}_{l_{1}-1-j, K \backslash J}^{\left(g_{2}\right)}$
III.b and the marked edge is a bridge $\rightarrow \mathcal{T}_{j, l_{1}-1-j, K}^{(g-1)}$. 


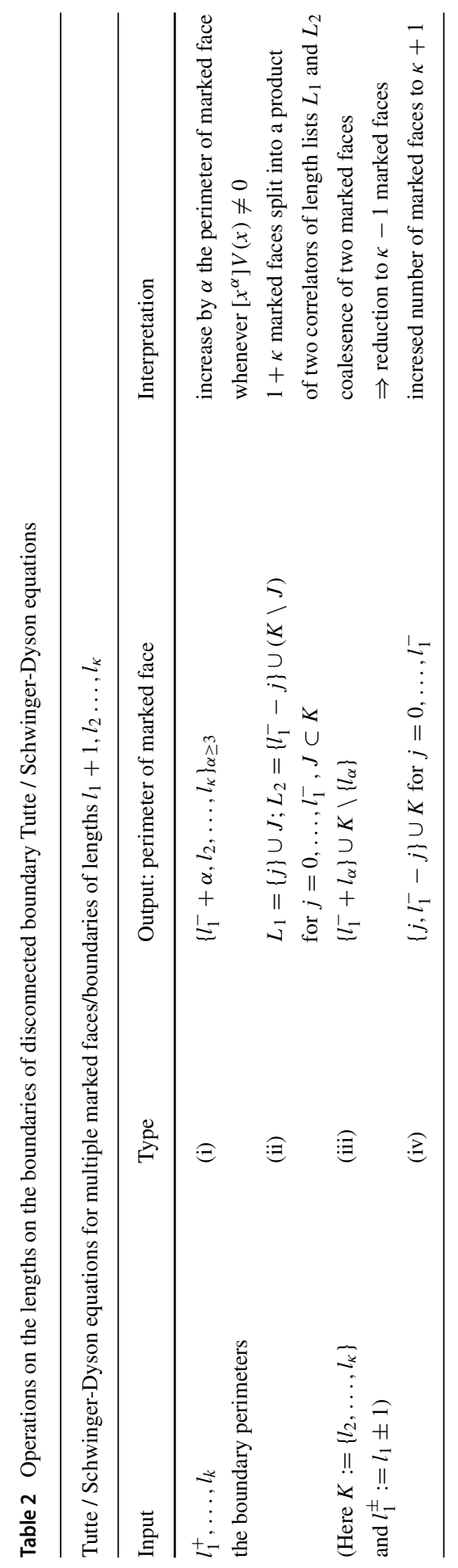


Table 3 Boundaries in both frameworks

Parallel between Tutte Equations and TFT SDE: Role of boundaries

\begin{tabular}{lll}
\hline Concept & Variable in Tutte Equations & Variable in TFT \\
\hline number of boundary components & $\kappa$ & $k$ \\
parameters of the boundaries & $\kappa$ lengths & $\begin{array}{l}D \text {-coloured graph } \mathcal{D} \\
\text { boundary decomposition }\end{array}$ \\
& $\left(l_{1}+1, l_{2}, \ldots, l_{\kappa}\right)$ & $\mathcal{D}=\mathcal{R} \sqcup Q_{1}^{\sqcup \alpha_{1}} \sqcup \ldots \sqcup Q_{n}^{\sqcup \alpha_{n}}$ \\
& & $\left(k=\alpha_{1}+\ldots+\alpha_{n}+1\right)$ \\
distinguished boundary component & $l_{1}+1$ & $\mathcal{R}$ \\
in the derivation of the equations & & (cf. Notation of the theorem) \\
\hline
\end{tabular}

Table 4 Tensor field theory analogue of Table 2. The notation in the column 'Term in SDE' refers each row in the (4.4). In types 2 through $4, \Delta L$ refers to the line of (4.4) containing a term of the form $[L(\mathbf{X})-$ $\left.L\left(\left.\mathbf{X}\right|_{s_{c} \rightarrow \xi}\right)\right]$ that can be easily read off (together with its factors and sums over automorphisms and, as appropriate, over momenta). The result of these operations for concrete graphs appears in Section 6

Disconnected-ə Schwinger Dyson Equations

\begin{tabular}{|c|c|c|}
\hline Term in SDE & Type & Transformation of $\mathcal{D}$ and interpretation \\
\hline$G_{\mathcal{D}}$ & 0. & None, $\mathcal{D}$ is input \\
\hline $\mathfrak{f}_{\mathcal{D}, s_{c}}^{(c)}$ & 1. & $\begin{array}{l}\text { Contraction of three vertices of } c \mathbb{g}^{c} \text { with }(2+\# \text { Vertices } \mathcal{D}) \text {-pt } \\
\text { functions, effectively leading to a boundary graph } \mathcal{D}\end{array}$ \\
\hline$\Delta\left(\frac{\partial W}{\partial S_{c}(\mathcal{D} ; \beta, \rho)}\right)$ & $2 \mathrm{~A}$. & $\begin{array}{l}\text { Contraction of two white vertices of } c_{g c} \text { with } \zeta_{c}(\mathcal{D} ; \beta, \rho) \\
\text { at the black vertices } \beta \text { and } \rho \Rightarrow \text { effectively } \mathcal{D} ; \\
\text { accounts for splitting and merging of boundaries. } \\
\text { Here } \rho \text { is a vertex of the graph } \mathcal{R}\end{array}$ \\
\hline $\boldsymbol{\Delta}\left(\frac{\partial W}{\partial \zeta_{c}(\mathcal{D} ; \beta, \rho)}\right)$ & $2 \mathrm{~B}$. & Idem, but $\rho$ is not a vertex of the graph $\mathcal{R}$ \\
\hline$\Delta G_{\mathcal{D}}$ & 3. & $\begin{array}{l}\text { The tadpole-like term contracting both (say) lower vertices of } \\
8 \subset \mathbb{C} \text { with two vertices of } \mathcal{D} \text { corresponding to } \mathbf{s} \text { and the white } \\
\text { vertex of } \mathcal{D} \text { connected to } \mathbf{s} \text { by a } c \text {-coloured edge }\end{array}$ \\
\hline$\Delta H_{\mathcal{B}, \mathcal{C}}^{(c, \tau)}$ & 4. & $\begin{array}{l}\text { For } \tau \in \mathrm{Br}^{(2)}(\mathcal{R}, \beta, c) \text {, splitting of } \mathcal{R} \text { into two connected } \\
\text { components, } \mathcal{R}^{\prime} \text { and } \mathcal{R}^{\prime \prime} ; \text { splitting of the remaining } \\
\text { components } \mathcal{B}, \mathcal{C} \text { of } \mathcal{D} \stackrel{\mathcal{c}}{\sim}(\mathcal{B}|\mathcal{C}| \mathcal{R}) \text {, as product } \\
\text { of correlators, } G_{\mathcal{R}^{\prime} \mid \mathcal{B}} \times G_{\mathcal{R}^{\prime \prime} \mid \mathcal{C}} \text {, one containing } \mathcal{R}^{\prime} \text {, the other } \mathcal{R}^{\prime \prime}\end{array}$ \\
\hline$I_{\mathcal{B}, \mathcal{C}}^{(c)}$ & 5. & $\begin{array}{l}\text { Splitting into product of correlators, one with an insertion } \\
\text { of } \delta \mathcal{C}, \text { namely } \mathfrak{f}_{\mathcal{C}, s_{c}}^{(c)} \text {, and } G_{\mathcal{R} \mid \mathcal{B}} \text {; altogeteher the components } \\
\text { of } \mathcal{R}, \mathcal{C}, \mathcal{B} \text { must be exactly those of } \mathcal{D}\end{array}$ \\
\hline
\end{tabular}


choice of vertex $\mathbf{S}$ does matter in the sense that different choices unrelated by nontrivial graph automorphisms of the distinguished boundary $\mathcal{R}$ yield different SDE.

\section{Four and Six Point SDE with Disconnected Boundary}

Concrete SDE's for the rank-3 $\varphi_{3}^{4}$-theory are presented next. Recall, the interaction in this case is $\lambda\left(1 \mathcal{L}_{1}+2 g_{2}+3 \mathscr{L}^{3}\right)$. We display some of the equations in traditional notation with explicit graphs, which allows to see immediately the graph operations. In other equations we use the simplification clarified in Table 6.

\subsection{Schwinger-Dyson Equations for $G^{(6)}$}

We single out the terms in the derivation of the SDE for this case, which is the most complicated presented here. The rest of the results are obtained in a similar and more direct way.

There are two equations, depending on whether one chooses $\mathbf{s}$ (cf. Theorem 1 above) as a component of the outgoing momentum in $\theta$ or in $i$ I $i$. We choose this last vertex to be $V_{1}=1$ ig 1 , for sake of clarity (since the model is colour-invariant, SDE for the other colours are readily obtained from it).

- If $\mathbf{s}=\mathbf{x}$ is outgoing momentum of the graph $\ominus$. In the notation of the theorem, here $\mathcal{D}=\mathrm{m} \mid V_{1}$ being $\mathcal{R}=\mathrm{m}$, since $\mathbf{y}^{1}=\mathbf{s}=X_{0}$ is the momentum of the black vertex of $\mathrm{m}$. Therefore $\mathcal{Q}=V_{1}$. The remaining momenta $\mathbb{X}$ equal $(\mathbf{y}, \mathbf{z})$.

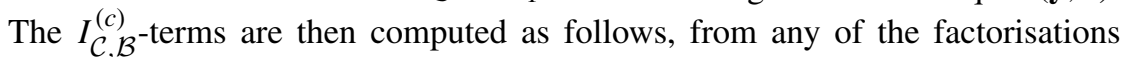
$\mathcal{C}, \mathcal{B}=\left(\varnothing, V_{1}\right)$ or $\left(V_{1}, \varnothing\right)$ and read

$$
\begin{aligned}
I_{\mathcal{C}, \mathcal{B}}^{(c)}(\mathbb{X}) & =\frac{1}{\left|\operatorname{Aut}_{c}(\mathcal{B})\right|}\left[\Theta_{V_{1}}\left(\mathfrak{f}_{\mathcal{C} ; s_{c}}^{(c)} \times G_{\mathrm{m} \mid \mathcal{B}}\left(X_{0} ; \bullet\right)\right)\right](\mathbb{X}) \\
& = \begin{cases}\mathfrak{f}_{V_{1} ; s_{c}}^{(c)}(\mathbf{y}, \mathbf{z}) G_{\mathrm{m}}^{(2)}(\mathbf{x}) & (\mathcal{C}, \mathcal{B})=\left(V_{1}, \varnothing\right), \\
\frac{1}{2} \mathfrak{f}_{\varnothing ; s_{c}}^{(c)} G_{\mathrm{m} \mid V_{1}}^{(6)}(\mathbf{x}, \mathbf{y}, \mathbf{z}) & (\mathcal{C}, \mathcal{B})=\left(\varnothing, V_{1}\right) .\end{cases}
\end{aligned}
$$

These correspond to the 'type 5' graph operation in Table 4. On these functions $\hat{\sigma} \in \operatorname{Aut}_{\mathrm{c}}\left(V_{1}\right)$ acts exchanging $\mathbf{y}$ with $\mathbf{z}$; just as on the terms coming from the derivative of the $Y$-term with respect to $\left(\mathrm{m} \mid V_{1}\right)$ :

$$
\sum_{\hat{\sigma} \in \mathbb{Z}_{2}} \sigma \cdot \mathfrak{f}_{\mathrm{m} \mid V_{1} ; x_{c}}^{(c)}(\mathbf{x}, \mathbf{y}, \mathbf{z})=\mathfrak{f}_{\mathrm{m} \mid V_{1} ; x_{c}}^{(c)}(\mathbf{x}, \mathbf{y}, \mathbf{z})+\mathfrak{f}_{\mathrm{m} \mid V_{1} ; x_{c}}^{(c)}(\mathbf{y}, \mathbf{x}, \mathbf{z})
$$




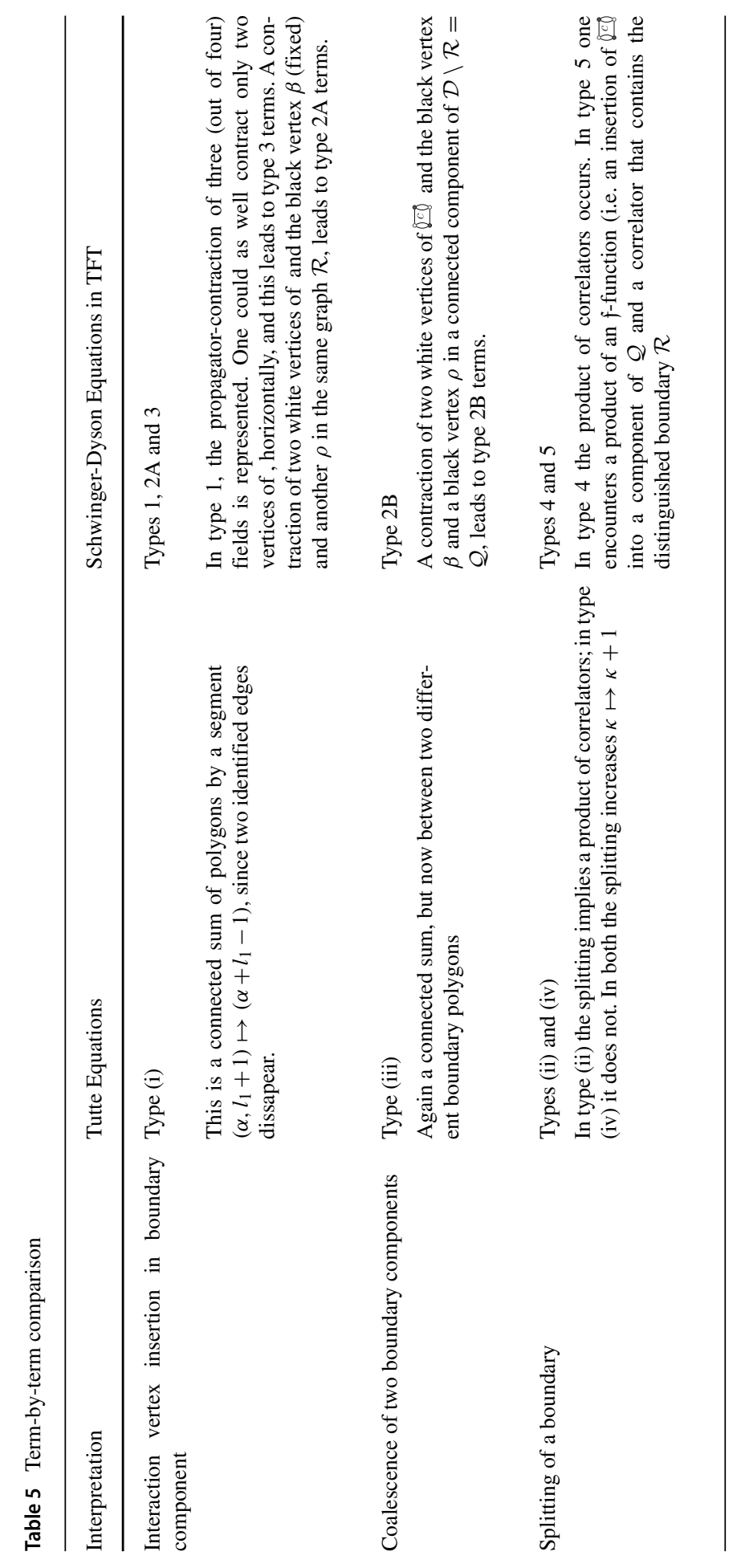


Table 6 Two notations for the correlation functions. Here $a, b, c$ are colours bound to satisfy $\{a, b, c\}=$ $\{1,2,3\}$. The subindex m originates from 'melon'

\section{CORRELATION FUNCTIONS}

\begin{tabular}{|c|c|c|}
\hline Order & Graph notation & Simplified notation \\
\hline 2-pt function & $G_{\ominus}^{(2)}$ & $G_{\mathrm{m}}^{(2)}$ or $G^{(2)}$ \\
\hline 4-pt functions & $\begin{array}{l}G_{a \rho j a}^{(4)} \\
G_{\ominus \mid \ominus}^{(4)}\end{array}$ & $\begin{array}{l}G_{V_{a}}^{(4)} \\
G_{\mathrm{m} \mid \mathrm{m}}^{(4)}\end{array}$ \\
\hline 6-pt functions & 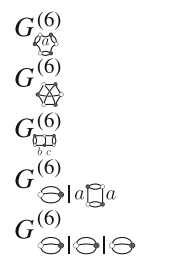 & $\begin{array}{l}G_{Q_{a}}^{(6)} \\
G_{K_{3,3}}^{(6)} \\
G_{F_{a ; b c}}^{(6)} \\
G_{\mathrm{m} \mid V_{a}}^{(6)} \\
G_{\mathrm{m}|\mathrm{m}| \mathrm{m}}^{(6)}\end{array}$ \\
\hline
\end{tabular}

Next, we obtain the second line in (4.4), i.e. the 'type 2 ' term in Table 4). This swap operation was pictured in Fig. 1. We have chosen $\mathbf{y}^{1}=\mathbf{s}(\beta=1)$, so

$$
\begin{gathered}
\frac{\partial W[J, \bar{J}]}{\partial \varsigma_{c=1}\left(\mathrm{~m} \mid V_{1} ; \beta=1, \rho=2,3\right)}(\mathbf{x}, \mathbf{y}, \mathbf{z})=G_{Q_{1}}^{(6)}(\mathbf{x}, \mathbf{y}, \mathbf{z}) \\
\frac{\partial W[J, \bar{J}]}{\partial \varsigma_{c=2}\left(\mathrm{~m} \mid V_{1} ; \beta=1, \rho=2\right)}(\mathbf{x}, \mathbf{y}, \mathbf{z})=G_{F_{3 ; 21}}^{(6)}(\mathbf{x}, \mathbf{z}, \mathbf{y}) \\
\frac{\partial W[J, \bar{J}]}{\partial \varsigma_{c=2}\left(\mathrm{~m} \mid V_{1} ; \beta=1, \rho=3\right)}(\mathbf{x}, \mathbf{y}, \mathbf{z})=G_{F_{3 ; 21}}^{(6)}(\mathbf{x}, \mathbf{y}, \mathbf{z}) \\
\frac{\partial W[J, \bar{J}]}{\partial \varsigma_{c=3}\left(\mathrm{~m} \mid V_{1} ; \beta=1, \rho=2\right)}(\mathbf{x}, \mathbf{y}, \mathbf{z})=G_{F_{2 ; 31}}^{(6)}(\mathbf{x}, \mathbf{z}, \mathbf{y}) \\
\frac{\partial W[J, \bar{J}]}{\partial \varsigma_{c=2}\left(\mathrm{~m} \mid V_{1} ; \beta=1, \rho=3\right)}(\mathbf{x}, \mathbf{y}, \mathbf{z})=G_{F_{2 ; 31}}^{(6)}(\mathbf{x}, \mathbf{y}, \mathbf{z})
\end{gathered}
$$

In this case the set $\operatorname{Br}^{(2)}(, \rho, c)$ is empty, for any values of $\rho$ and $c$ (thus no terms of 'type 4' appear, cf. Table 4). Therefore, the sum over the $H$-terms vanishes. For each $(\mathbf{x}, \mathbf{y}, \mathbf{z}) \in \mathcal{F}_{3,3}$,

$$
\begin{aligned}
& G_{\mathrm{m} \mid V_{1}}^{(6)}(\mathbf{x}, \mathbf{y}, \mathbf{z}) \\
= & \left(\frac{-2 \lambda}{E_{\mathbf{x}}}\right) \times\left\{\sum_{c=1}^{3} \mathfrak{f}_{\mathrm{m} \mid V_{1} ; x_{c}}^{(c)}(\mathbf{x}, \mathbf{y}, \mathbf{z})+\mathfrak{f}_{\mathrm{m} \mid V_{1} ; x_{c}}^{(c)}(\mathbf{y}, \mathbf{x}, \mathbf{z})\right. \\
+ & \frac{1}{E\left(y_{1}, x_{1}\right)}\left[G_{Q_{1}}^{(6)}(\mathbf{x}, \mathbf{y}, \mathbf{z})-G_{Q_{1}}^{(6)}\left(y_{1}, x_{2}, x_{3}, \mathbf{y}, \mathbf{z}\right)\right] \\
+ & \frac{1}{E\left(z_{1}, x_{1}\right)}\left[G_{Q_{1}}^{(6)}(\mathbf{x}, \mathbf{y}, \mathbf{z})-G_{Q_{1}}^{(6)}\left(z_{1}, x_{2}, x_{3}, \mathbf{y}, \mathbf{z}\right)\right]
\end{aligned}
$$




$$
\begin{aligned}
& +\frac{1}{E\left(z_{2}, x_{2}\right)}\left[G_{F_{3 ; 21}}(\mathbf{x}, \mathbf{z}, \mathbf{y})-G_{F_{3 ; 21}}\left(x_{1}, z_{2}, x_{3}, \mathbf{z}, \mathbf{y}\right)\right] \\
& +\frac{1}{E\left(y_{2}, x_{2}\right)}\left[G_{F_{3 ; 21}}(\mathbf{x}, \mathbf{y}, \mathbf{z})-G_{F_{3 ; 21}}\left(x_{1}, y_{2}, x_{3} \mathbf{y}, \mathbf{z}\right)\right] \\
& +\frac{1}{E\left(z_{3}, x_{3}\right)}\left[G_{F_{2 ; 31}}(\mathbf{x}, \mathbf{z}, \mathbf{y})-G_{F_{2 ; 31}}\left(x_{1}, x_{2}, z_{3}, \mathbf{z}, \mathbf{y}\right)\right] \\
& +\frac{1}{E\left(y_{3}, x_{3}\right)}\left[G_{F_{2 ; 31}}(\mathbf{x}, \mathbf{y}, \mathbf{z})-G_{F_{2 ; 31}}\left(x_{1}, x_{2}, y_{3} \mathbf{y}, \mathbf{z}\right)\right] \\
& -\sum_{c=1}^{3}\left[\sum_{b_{c}} \frac{1}{E\left(x_{c}, b_{c}\right)}\left[G_{\mathrm{m} \mid V_{1}}^{(6)}(\mathbf{x}, \mathbf{y}, \mathbf{z})-G_{\mathrm{m} \mid V_{1}}^{(6)}\left(\mathbf{x}_{\hat{c}} b_{c}, \mathbf{y}, \mathbf{z}\right)\right]\right. \\
& \left.+\left[\left(\mathfrak{f}_{V_{1} ; s_{c}}^{(c)}(\mathbf{z}, \mathbf{y})+\mathfrak{f}_{V_{1} ; s_{c}}^{(c)}(\mathbf{y}, \mathbf{z})\right] \cdot G^{(2)}(\mathbf{x})+\mathfrak{f}_{\varnothing ; s_{c}}^{(c)} G_{\mathrm{m} \mid V_{1}}^{(6)}(\mathbf{x}, \mathbf{y}, \mathbf{z})\right]\right\} .
\end{aligned}
$$

In the second line after the curly bracket 'type 1' terms (derived from the $Y$-term) appear. Also, The second to last line consists of 'type 3' terms.

- If $\mathbf{s}=\mathbf{x}$ is outgoing momentum of the boundary graph ${ }^{1} \mathrm{~g}_{1}$. For $\mathbf{s}=\left(x_{1}, y_{2}, y_{3}\right)$ an outgoing momentum of $V_{1}$ we derive now the SDE for $G_{\mathrm{m} \mid V_{1}}^{(6)}$. Then $\mathcal{R}=V_{1}$, $\mathcal{Q}=\mathrm{m}$, by definition. The $I_{\mathcal{C}, \mathcal{B}}^{(c)}$-coefficients are given by

$$
\begin{aligned}
I_{\mathrm{m}, \varnothing}^{(c)}(\mathbf{x}, \mathbf{y}, \mathbf{z}) & =\mathfrak{f}_{\mathrm{m} ; s_{c}}^{(c)}(\mathbf{z}) \cdot G_{V_{1}}(\mathbf{x}, \mathbf{y}), \\
I_{\varnothing, \mathrm{m}}^{(c)}(\mathbf{x}, \mathbf{y}, \mathbf{z}) & =\mathfrak{f}_{\varnothing ; s_{c}}^{(c)} \cdot G_{V_{1} \mid \mathrm{m}}(\mathbf{x}, \mathbf{y}, \mathbf{z}) .
\end{aligned}
$$

The $H_{\mathcal{C}, \mathcal{B}}^{(c, \tau)}$-terms are computed from the set

$$
\operatorname{Br}^{(2)}\left(1 \mathcal{L}^{1}, \beta=1, c\right)= \begin{cases}\{2\} & c=1, \\ \varnothing & \text { otherwise },\end{cases}
$$

since only the colour-1 swap at the vertex $\mathbf{s}$ with the vertex with outgoing momentum $\mathbf{y}^{2}$ (also in $V_{1}$ ) separates $1 \mathcal{L}^{1}$. The only contributions are therefore

$$
\begin{aligned}
& H_{\varnothing, \mathrm{m}}^{(c=1, \tau=2)}(\mathbf{X})=G^{(2)}(\mathbf{x}) \cdot G_{\mathrm{m} \mid \mathrm{m}}^{(4)}(\mathbf{y}, \mathbf{z}), \\
& H_{\mathrm{m}, \varnothing}^{(c=1, \tau=2)}(\mathbf{X})=G_{\mathrm{m} \mid \mathrm{m}}^{(4)}(\mathbf{x}, \mathbf{z}) \cdot G^{(2)}(\mathbf{y}),
\end{aligned}
$$

(which correspond to 'type 4' terms, cf. Table 4). Thus $G_{V_{1} \mid \mathrm{m}}^{(6)}$ satisfies, for all $(\mathbf{x}, \mathbf{y}, \mathbf{z}) \in \mathcal{F}_{3,3}$

$$
\begin{aligned}
& G_{V_{1} \mid \mathrm{m}}^{(6)}(\mathbf{x}, \mathbf{y}, \mathbf{z}) \\
= & \left(\frac{-2 \lambda}{E_{\mathbf{s}}}\right) \times\left\{\sum_{c=1}^{3} \mathfrak{f}_{\mathrm{m} \mid V_{1} ; s_{c}}^{(c)}(\mathbf{z}, \mathbf{x}, \mathbf{y})+\mathfrak{f}_{\mathrm{m} \mid V_{1} ; s_{c}}^{(c)}(\mathbf{z}, \mathbf{y}, \mathbf{x})\right. \\
+ & \frac{1}{E\left(y_{1}, x_{1}\right)}\left[G_{\mathrm{m}|\mathrm{m}| \mathrm{m}}^{(6)}(\mathbf{x}, \mathbf{y}, \mathbf{z})-G_{\mathrm{m}|\mathrm{m}| \mathrm{m}}^{(6)}\left(y_{1}, x_{2}, x_{3}, \mathbf{y}, \mathbf{z}\right)\right] \\
+ & \frac{1}{E\left(z_{1}, x_{1}\right)}\left[G_{Q_{1}}^{(6)}(\mathbf{x}, \mathbf{y}, \mathbf{z})-G_{Q_{1}}^{(6)}\left(z_{1}, x_{2}, x_{3} ; \mathbf{y}, \mathbf{z}\right)\right]
\end{aligned}
$$




$$
\begin{aligned}
& +\frac{1}{E\left(x_{2}, y_{2}\right)}\left[G_{V_{3} \mid \mathrm{m}}^{(6)}(\mathbf{x}, \mathbf{y}, \mathbf{z})-G_{V_{3} \mid \mathrm{m}}^{(6)}\left(x_{1}, y_{2}, x_{3} ; \mathbf{y}, \mathbf{z}\right)\right] \\
& +\frac{1}{E\left(z_{2}, y_{2}\right)}\left[G_{F_{3 ; 12}}^{(6)}(\mathbf{x}, \mathbf{y}, \mathbf{z})-G_{F_{3 ; 12}}^{(6)}\left(\mathbf{x} ; y_{1}, x_{2}, y_{3} ; \mathbf{z}\right)\right] \\
& +\frac{1}{E\left(x_{3}, y_{3}\right)}\left[G_{V_{2} \mid \mathrm{m}}^{(6)}(\mathbf{x}, \mathbf{y}, \mathbf{z})-G_{V_{2} \mid \mathrm{m}}^{(6)}\left(\mathbf{x} ; y_{1}, y_{2}, x_{3} ; \mathbf{z}\right)\right] \\
& +\frac{1}{E\left(z_{3}, y_{3}\right)}\left[G_{F_{2 ; 13}}^{(6)}(\mathbf{x}, \mathbf{y}, \mathbf{z})-G_{F_{2 ; 13}}^{(6)}\left(\mathbf{x} ; y_{1}, y_{2}, z_{3} ; \mathbf{z}\right)\right] \\
& -\sum_{c=1}^{3}\left[\sum_{b_{c}} \frac{1}{E\left(s_{c}, b_{c}\right)}\left[G_{V_{1} \mid \mathrm{m}}^{(6)}(\mathbf{x}, \mathbf{y}, \mathbf{z})-G_{V_{1} \mid \mathrm{m}}^{(6)}\left(\left.[\mathbf{x}, \mathbf{y}, \mathbf{z}]\right|_{s_{c} \rightarrow b_{c}}\right)\right]\right. \\
& \left.+\left[\left(\mathfrak{f}_{V_{1} ; s_{c}}^{(c)}(\mathbf{z}, \mathbf{y})+\mathfrak{f}_{V_{1} ; s_{c}}^{(c)}(\mathbf{y}, \mathbf{z})\right] \cdot G^{(2)}(\mathbf{x})+\mathfrak{f}_{\varnothing ; s_{c}}^{(c)} G_{\mathrm{m} \mid V_{1}}^{(6)}(\mathbf{x}, \mathbf{y}, \mathbf{z})\right]\right\} .
\end{aligned}
$$

\subsection{Schwinger-Dyson Equation for $G_{\ominus}^{(4)}$}

There is only one SDE for the 'disconnected- $\partial$ ' 4-point function. For every $(\mathbf{x}, \mathbf{y}, \mathbf{z}) \in$ $\mathcal{F}_{3,3}$,

$$
\begin{aligned}
& G_{\ominus \mid \ominus}^{(4)}(\mathbf{x}, \mathbf{y}) \\
& =\left(\frac{-2 \lambda}{E_{\mathbf{x}}}\right) \times \sum_{c=1}^{3}\left\{\sum_{\mathbf{q}_{\hat{c}}} G_{\ominus}^{(2)}\left(x_{c}, \mathbf{q}_{\hat{c}}\right) \cdot G_{\ominus \mid \ominus}^{(4)}(\mathbf{x}, \mathbf{y})+G_{\ominus}^{(2)}(\mathbf{x}) \mathfrak{f}_{\ominus, x_{c}}^{(c)}(\mathbf{y})\right. \\
& +\sum_{b_{c}} \frac{1}{E\left(b_{c}, x_{c}\right)}\left[G_{\ominus \mid \ominus}^{(4)}(\mathbf{x}, \mathbf{y})-G_{\ominus \mid \ominus}^{(4)}\left(b_{c} \mathbf{x}_{\hat{c}}, \mathbf{y}\right)\right] \\
& +\frac{1}{E\left(y_{c}, x_{c}\right)}\left[G_{c \mid g_{c}}^{(4)}(\mathbf{x}, \mathbf{y})-G_{c \mid g c}^{(4)}\left(b_{c} \mathbf{x}_{\hat{c}}, \mathbf{y}\right)\right] \\
& \left.+\mathfrak{f}_{x_{c}, \vartheta \mid \ominus}^{(c)}(\mathbf{x}, \mathbf{y})+\mathfrak{f}_{x_{c}, \vartheta \mid \ominus}^{(c)}(\mathbf{y}, \mathbf{x})\right\} \text {. }
\end{aligned}
$$

Only this equation is not new, but was already (directly) derived in [50], in notation of Table 6. 


\subsection{Schwinger-Dyson Equation for $\boldsymbol{G}_{\ominus}^{(6)}|\ominus| \ominus$}

Similarly, since one can permute the arguments of $G_{\mathrm{m}|\mathrm{m}| \mathrm{m}}^{(6)}$, it satisfies only one SDE:

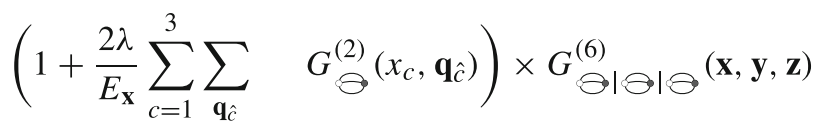

$$
\begin{aligned}
& =\left(\frac{-2 \lambda}{E_{\mathbf{x}}}\right) \sum_{c=1}^{3}\left\{\mathfrak{f}_{\partial ; x_{c}}^{(c)}(\mathbf{y}) G_{\partial|\vartheta|}^{(4)}(\mathbf{x}, \mathbf{z})+\mathfrak{f}_{\partial ; x_{c}}^{(c)}(\mathbf{z}) G_{\partial|\partial|}^{(4)}(\mathbf{x}, \mathbf{y})\right. \\
& +G_{\ominus}^{(2)}(\mathbf{x}) \cdot \mathfrak{f}_{\ominus \mid \ominus}^{(c)}(\mathbf{y}, \mathbf{z}) \\
& -\sum_{b_{c}} \frac{1}{E\left(x_{c}, b_{c}\right)}\left[G_{\ominus|\ominus| \ominus}^{(6)}(\mathbf{x}, \mathbf{y}, \mathbf{z})-G_{\ominus|\ominus| \ominus}^{(6)}\left(b_{c} \mathbf{x}_{\hat{c}}, \mathbf{y}, \mathbf{z}\right)\right] \\
& +\frac{1}{E\left(y_{c}, x_{c}\right)}\left[G_{\ominus|\delta c \emptyset|}^{(6)}(\mathbf{z}, \mathbf{x}, \mathbf{y})-G_{\ominus|\delta c \emptyset|}^{(6)}\left(\mathbf{z}, y_{c} \mathbf{x}_{\hat{c}}, \mathbf{y}\right)\right] \\
& +\frac{1}{E\left(z_{c}, x_{c}\right)}\left[G_{\ominus|\emptyset c|}^{(6)}(\mathbf{y}, \mathbf{x}, \mathbf{z})-G_{\ominus|\delta c \emptyset|}^{(6)}\left(\mathbf{y}, y_{c} \mathbf{x}_{\hat{c}}, \mathbf{z}\right)\right] \\
& \left.+\sum_{\sigma \in \mathfrak{S}(3)} \sigma \cdot \mathfrak{f}_{\ominus}^{(c)} \theta(\mathbf{x}, \mathbf{y}, \mathbf{z})\right\} \text {. }
\end{aligned}
$$

We kept the graph notation in order to ease the reading of the graph movements. Equivalently,

$$
\begin{aligned}
\left(1+\frac{2 \lambda}{E_{\mathbf{x}}} \sum_{c=1}^{3} \sum_{\mathbf{q}_{\hat{c}}}\right. & \left.G^{(2)}\left(x_{c}, \mathbf{q}_{\hat{c}}\right)\right) \times G_{\mathrm{m}|\mathrm{m}| \mathrm{m}}^{(6)}(\mathbf{x}, \mathbf{y}, \mathbf{z}) \\
= & \left(\frac{-2 \lambda}{E_{\mathbf{x}}}\right) \sum_{c=1}^{3}\left\{\mathfrak{f}_{\mathrm{m} ; x_{c}}^{(c)}(\mathbf{y}) G_{\mathrm{m} \mid \mathrm{m}}^{(4)}(\mathbf{x}, \mathbf{z})+\mathfrak{f}_{\mathrm{m} ; x_{c}}^{(c)}(\mathbf{z}) G_{\mathrm{m} \mid \mathrm{m}}^{(4)}(\mathbf{x}, \mathbf{y})\right. \\
+ & G_{\ominus}^{(2)}(\mathbf{x}) \cdot \mathfrak{f}_{\mathrm{m} \mid \mathrm{m}}^{(c)}(\mathbf{y}, \mathbf{z}) \\
& -\sum_{b_{c}} \frac{1}{E\left(x_{c}, b_{c}\right)}\left[G_{\mathrm{m}|\mathrm{m}| \mathrm{m}}^{(6)}(\mathbf{x}, \mathbf{y}, \mathbf{z})-G_{\mathrm{m}|\mathrm{m}| \mathrm{m}}^{(6)}\left(b_{c} \mathbf{x}_{\hat{c}}, \mathbf{y}, \mathbf{z}\right)\right] \\
& +\frac{1}{E\left(y_{c}, x_{c}\right)}\left[G_{\mathrm{m} \mid V_{c}}^{(6)}(\mathbf{z}, \mathbf{x}, \mathbf{y})-G_{\mathrm{m} \mid V_{c}}^{(6)}\left(\mathbf{z}, y_{c} \mathbf{x}_{\hat{c}}, \mathbf{y}\right)\right] \\
& +\frac{1}{E\left(z_{c}, x_{c}\right)}\left[G_{\mathrm{m} \mid V_{c}}^{(6)}(\mathbf{y}, \mathbf{x}, \mathbf{z})-G_{\mathrm{m} \mid V_{c}}^{(6)}\left(\mathbf{y}, y_{c} \mathbf{x}_{\hat{c}}, \mathbf{z}\right)\right] \\
& +\sum_{\left.\sigma \in \mathfrak{S}_{(3)} \sigma \cdot \mathfrak{f}_{\mathrm{m}|\mathrm{m}| \mathrm{m}}^{(c)}(\mathbf{x}, \mathbf{y}, \mathbf{z})\right\}}
\end{aligned}
$$




\section{Conclusions and Outlook}

We introduced the multi-variable graph calculus - a tool to prove a general formula for the disconnected- $\partial$ Schwinger-Dyson for the most general quartic-melonic tensor field theory in arbitrary rank (Theorem 1). In their description, a set of graph operations on an input graph has been exposed in Table 4. This list is the tensorial equivalent of the matrix model operations on the boundary graphs of matrix models (cf. Table 2). This exposition is focused on bipartite coloured graphs (a generalization addressing those graphs required by real $\mathrm{O}(N)^{D}$-invariant tensor models [20] would be interesting). Some of these operations are not new, but appear already in the SDE of tensor models (in a tree language [30]; see also [37])

The well-known dictionary between the theory of enumeration of random maps and matrix models allows to pose two uses our SDE could be useful for. The theories of graph-encoded manifolds [21, 39, 55] bring tensor models into prominence as a theory of 'random higher-dimensional maps'. For instance, gluings of octahedra [13] are studied from the tensor model with (non-melonic) interaction vertex

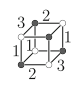

which is the dual graph-representation to an octahedron. The boundary-completeness of the quartic-melonic models [53, Thm 1] further studied here shows a path to the theory of higher-dimensional maps made of gluings of particular triangulations of $D$-balls, in the rank- $D$ case. The natural candidate for higher-dimensional Tutte equations is the set of relations derived from the $1 / N$-sectors of the present SDE that have the same boundary as well as value of Gurău degree. This is based on the fact (recalled in Section 5) that Tutte equations hide in the $N^{2-2 g-\kappa}$-sectors of Migdal's SDE for matrix models.

The second guess is the existence of a recursion allowing to compute higher-point TFT-correlators from some small number of lower-point ones, analogous to the Topological Recursion (TR) [3, 15, 24, 25, 60] that satisfy matrix model correlators. What is not speculative is that, assuming the dictionary of last paragraph, such tensorial TR would require the disconnected- $\partial$ SDE, since it is also a recursion in the number of boundaries (presumably also in their Gurău's degree). The blobbed TR for quartic tensor models has already been obtained [5] —yet it would be interesting to develop the purely tensorial ${ }^{20}$ cousin of the TR for tensor field theory ${ }^{21}$.

The large- $N$ limit of the disconnected- $\partial$ SDE should be analysed in order to access also their physical significance. At leading order, their melonic approximation [47] is expected to yield closed equations. A significant progress in this direction has been

\footnotetext{
${ }^{20}$ The Bonzom-Dartois TR bases on an initial Hubbard-Stratonovich transformation. Vertically cutting the pillow vertices, as the rank-3 $c g_{c}$, they "map" quartic melonic tensor models to a suitable intermediate field multi-matrix model, for which the authors develop a TR analogous to the introduced by Borot [14].

${ }^{21}$ The difference between tensor models and tensor field theory is here substantial. The former usually focuses on numerical observables $\left\langle\operatorname{Tr}_{\mathcal{B}}(\varphi, \bar{\varphi})\right\rangle \in \mathbb{C}$ and the latter on functions (or distributions) $G_{\mathcal{B}}$ : $\mathscr{V}(\mathcal{B}) \rightarrow \mathbb{C}$. In contrast, the loop equations, Ward Identities [34] and SDE [30, 31] are algebraic in the for tensor models, whereas for tensor field theory 'loop equations' [51,53] are integro-differential, as shown also in [50] explicitly.
} 
undertaken in [50], whose techniques could be extended to the present disconnected$\partial \mathrm{SDE}$ in a next project.

Heading towards a quantum gravity perspective, objects appearing in the Functional Renormalization Group [8] —or Ward-constrained flows [40, 41] — are expected to be described in terms of graph-generated functionals studied here. Together with the boundary-completeness of the quartic-melonic models [53, Thm 1], this motivates us to study the geometric nature of the flow from a simple quartic model.

Using the bordism interpretation [53] of the correlation functions, the WheelerdeWitt equation ${ }^{22}$ claimed [59] to be formally satisfied by the wave function $\Psi(q) \sim$ $\int_{\partial g=q} \mathcal{D}[g] \mathrm{e}^{-S_{\text {Einstein-Hibert }}[g]}[33]$ for a boundary 3D geometry (with metric $q$ ) would be worth to explore in this context, probably guided by [6].

On the purely mathematical side, systems of graph-group actions can be extended to Lie groups actions and to calculi in infinitely many graph-variables by using rigorous analytic tools. It would be also interesting to consider the coefficient functions $u_{g}$ directly in certain algebra of functions. One could dispense with the functions $\{\mathscr{V}(g) \rightarrow \mathcal{C}\}$ by using instead directly (non-commutative) algebras.

Finally, the (symmetric) monoidal structure on the set of boundary graphs emerges in a natural way. This guides us towards the language of Topological Quantum Field Theories (TQFT) [4]. Since these boundary graphs triangulate boundary states, an interesting program would be to obtain discrete TQFT from matrix models and 'TQFT with observables' [45] from tensor models, or enframe these in Oeckl's positive boundary formalism (op. cit.), which also facilitates the gluing-boundary procedure that TQFT provides (likely informed by the GFT-treatement of Boulatov and Ooguri models with GFT in [19]). In the tensor and matrix models case, the gluing of boundaries should be implemented as an operation $\wedge_{\mathcal{A}}$ on two correlation functions sharing a boundary state $\mathcal{A}, G_{\mathcal{A}|\mathcal{B}| \cdots \mid \mathcal{C}^{\prime}} \wedge_{\mathcal{A}} G_{\mathcal{A}\left|\mathcal{B}^{\prime}\right| \cdots \mid \mathcal{C}^{\prime}}$, which should be related to $G_{\mathcal{B}\left|\mathcal{B}^{\prime}\right| \cdots|\mathcal{C}| \mathcal{C}^{\prime}}$ due to their geometrical interpretation.

Acknowledgements The author thanks Raimar Wulkenhaar and Adrian Tanasă for hospitality; and Romain Pascalie for helpful hints and carefully reading the draft (any error is the author's responsibility). Thanks to the Faculty of Physics, Astronomy and Applied Computer Science, Jagiellonian University (Cracow, Poland), where part of this article was written, and to Andrzej Sitarz as well for hospitality. The author acknowledges the Short-Term Scientific Mission program of the COST Action MP 1405 for this mobility opportunity. This research was funded by the Deutsche Forschungsgemeinschaft, SFB 878 (Mathematical Institute of the University of Münster, Germany). Subsequently it was carried out at the Institute of Theoretical Physics, University of Warsaw and has been supported by the TEAM programme of the Foundation for Polish Science co-financed by the European Union under the European Regional Development Fund (POIR.04.04.00-00-5C55/17-00).

Open Access This article is licensed under a Creative Commons Attribution 4.0 International License, which permits use, sharing, adaptation, distribution and reproduction in any medium or format, as long as you give appropriate credit to the original author(s) and the source, provide a link to the Creative Commons

\footnotetext{
${ }^{22}$ The author acknowledges one of the anonymous referees for this comment.
} 
licence, and indicate if changes were made. The images or other third party material in this article are included in the article's Creative Commons licence, unless indicated otherwise in a credit line to the material. If material is not included in the article's Creative Commons licence and your intended use is not permitted by statutory regulation or exceeds the permitted use, you will need to obtain permission directly from the copyright holder. To view a copy of this licence, visit http://creativecommonshorg/licenses/by/4.0/.

\section{Appendix A: The first coefficients of the $Y$-term}

For completeness, we give the first coefficients of the $Y$-term, keeping in mind the notation simplification (Table 6). The computation of these functions is presented in detail in [53]. As before, the set equality $\{a, b, c\}=\{1,2,3\}$ holds.

$$
\begin{aligned}
& \mathfrak{f}_{\mathrm{m} ; s_{a}}^{(a)}(\mathbf{x})=G_{V_{a}}^{(4)}\left(\mathbf{x}, s_{a}, x_{b}, x_{c}\right) \\
& +\sum_{c \neq a q_{b} \in \mathbb{Z}} G_{V_{c}}^{(4)}\left(\mathbf{x} ; s_{a}, q_{b}, x_{c}\right)+\sum_{q_{b}, q_{c}} G_{\mathrm{m} \mid \mathrm{m}}^{(4)}\left(\mathbf{x} ; s_{a}, q_{b}, q_{c}\right), \\
& \mathfrak{f}_{V_{a} ; s_{a}}^{(a)}(\mathbf{x}, \mathbf{y})=\frac{1}{3}\left(G_{Q_{a}}^{(6)}\left(s_{a}, x_{b}, x_{c}, \mathbf{x}, \mathbf{y}\right)+\operatorname{cyclic} \text { perm. in }\left(s_{a}, x_{b}, x_{c}\right), \mathbf{x} \text { and } \mathbf{x}\right) \\
& +\frac{1}{3}\left(G_{K_{3,3}}^{(6)}\left(s_{a}, x_{b}, y_{c} ; \mathbf{x}, \mathbf{y}\right)+\text { cyclic perm. }\right) \\
& +\sum_{q_{b}} G_{F_{b ; a c}}^{(6)}\left(\mathbf{x} ; \mathbf{y} ; s_{a}, q_{b}, y_{c}\right)+\sum_{q_{c}} G_{F_{c ; a b}}^{(6)}\left(\mathbf{x} ; \mathbf{y} ; s_{a}, q_{c}, y_{b}\right) \\
& +\frac{1}{2} \sum_{q_{b}, q_{c}} G_{\mathrm{m} \mid V_{a}}^{(6)}\left(s_{a}, q_{b}, q_{c} ; \mathbf{x} ; \mathbf{y}\right), \\
& \mathfrak{f}_{V_{b} ; s_{a}}^{(a)}(\mathbf{x}, \mathbf{y})=\frac{1}{3}\left(\sum_{q_{b}} G_{Q_{b}}^{(6)}\left(s_{a}, q_{b}, y_{c} ; \mathbf{x}, \mathbf{y}\right)+\text { cyclic perm. }\right)+G_{F_{c} ; a b}^{(6)}\left(s_{a}, y_{b}, x_{c} ; \mathbf{x} ; \mathbf{y}\right) \\
& +G_{F_{c ; a b}}^{(6)}\left(\mathbf{x} ; s_{a}, x_{b}, x_{c} ; \mathbf{y}\right)+\sum_{q_{b}} G_{F_{a ; b c}}^{(6)}\left(\mathbf{x} ; \mathbf{y} ; s_{a}, q_{b}, y_{c}\right) \\
& -\frac{1}{2} \sum_{q_{b}, q_{c}} G_{\mathrm{m} \mid V_{b}}^{(6)}\left(s_{a}, q_{b}, q_{c} ; \mathbf{x} ; \mathbf{y}\right), \\
& \mathfrak{f}_{\mathrm{m} \mid \mathrm{m} ; s_{a}}^{(a)}(\mathbf{x}, \mathbf{y})=\left(\sum_{q_{b}, q_{c}} G_{\mathrm{m}|\mathrm{m}| \mathrm{m}}^{(6)}\left(s_{a}, q_{b}, q_{c}, \mathbf{x}, \mathbf{y}\right)+\text { cyclic perm. }\right) \\
& +G_{\mathrm{m} \mid V_{a}}^{(6)}\left(\mathbf{x}, s_{a}, y_{b}, y_{c}, \mathbf{y}\right)+\sum_{q_{c}} G_{\mathrm{m} \mid V_{b}}^{(6)}\left(\mathbf{x}, s_{a}, y_{b}, q_{c}, \mathbf{y}\right) \\
& +\sum_{q_{b}} G_{\mathrm{m} \mid V_{c}}^{(6)}\left(\mathbf{x}, s_{a}, q_{b}, y_{c}, \mathbf{y}\right)+\sum_{q_{c}} G_{\mathrm{m} \mid V_{b}}^{(6)}\left(\mathbf{x}, \mathbf{y}, s_{a}, y_{b}, q_{c}\right) \\
& +\sum_{q_{b}} G_{\mathrm{m} \mid V_{c}}^{(6)}\left(\mathbf{x}, \mathbf{y}, s_{a}, q_{b}, y_{c}\right)+G_{\mathrm{m} \mid V_{a}}^{(6)}\left(\mathbf{x}, \mathbf{y}, s_{a}, y_{b}, y_{c}\right) \\
& +G_{F_{a ; b c}}^{(6)}\left(\mathbf{x}, s_{a}, x_{b}, y_{c}, \mathbf{y}\right) \text {. }
\end{aligned}
$$




\section{References}

1. Ambjørn, J., Durhuus, B., Jonsson, T.: Three-dimensional simplicial quantum gravity and generalized matrix models. Mod. Phys. Lett. A6, 1133-1146 (1991)

2. Ambjørn, J., Görlich, A., Jurkiewicz, J., Loll, R.: Causal dynamical triangulations and the search for a theory of quantum gravity. Int. J. Mod. Phys. D22, 1330019 (2013)

3. Andersen, J.E., Chekhov, L.O., Penner, R.C., Reidys, C.M., Sułkowski, P.: Topological recursion for chord diagrams, RNA complexes, and cells in moduli spaces. Nucl. Phys. B866, 414-443 (2013)

4. Atiyah, M.: Topological quantum field theories. Inst. Hautes Etudes Sci. Publ. Math. 68, 175-186 (1989)

5. Bonzom, V., Dartois, S.: Blobbed topological recursion for the quartic melonic tensor model. J. Phys. A51(32), 325201 (2018)

6. Bonzom, V., Freidel, L.: The Hamiltonian constraint in 3d Riemannian loop quantum gravity. Class. Quant. Grav. 195006, 28 (2011)

7. Geloun, J.B.: Asymptotic freedom of rank 4 tensor group field theory (2012)

8. Geloun, J.B., Koslowski, T.A., Oriti, D., Pereira, A.D.: Functional Renormalization Group analysis of rank 3 tensorial group field theory: The full quartic invariant truncation, vol. D97 (2018)

9. Geloun, J.B., Rivasseau, V.: A renormalizable 4-dimensional tensor field theory. Commun. Math. Phys. 318, 69-109 (2013)

10. Bonzom, V., Gurău, R., Riello, A., Rivasseau, V.: Critical behavior of colored tensor models in the large N limit. Nucl Phys. B853, 174-195 (2011)

11. Geloun, J.B., Samary, D.O.: 3D tensor field theory: renormalization and one-loop $\beta$-functions. Annales Henri Poincare 14, 1599-1642 (2013)

12. Brezin, É., Itzykson, C., Parisi, G., Zuber, J.-B.: Planar diagrams. Math. Commun. Phys. 59, 35 (1978)

13. Bonzom, V., Lionni, L.: Counting gluings of octahedra. Elec. J. Combin. 24 (2016)

14. Borot, G.: Blobbed topological recursion. Theor. Math. Phys. 185(3), 1729-1740 (2015). [Teor. Mat. Fiz.185,no.3,423(2015)]

15. Borot, G.: Lecture notes on topological recursion and geometry. arXiv:1705.09986 (2017)

16. Chamseddine, A.H., Connes, A.: The spectral action principle. Commun. Math. Phys. 186, 731-750 (1997)

17. Carrozza, S., Oriti, D., Rivasseau, V.: Renormalization of tensorial group field theories: abelian u(1) models in four dimensions. Commun. Math. Phys. 327, 603-641 (2014)

18. Carrozza, S., Pozsgay, V.: SYK-like tensor quantum mechanics with $\operatorname{Sp}(N)$ symmetry. Nucl. Phys. B 941, 28-52 (2019)

19. Carrozza, S.: Tensorial methods and renormalization in Group Field Theories. Phd Thesis, Orsay, LPT (2013)

20. Carrozza, S., Adrian, T.: $O(N)$ Random tensor models. Lett. Math. Phys. 106(11), 1531-1559 (2016)

21. Cassali, R., Cristofori, P.: Cataloguing PL 4-manifolds by gem-complexity. Electron. J. Combin. 22(4), 1-25 (2015). \#P4.25

22. Disertori, M., Gurău, R., Magnen, J., Rivasseau, V.: Vanishing of beta function of non commutative $\Phi_{4}^{4}$ theory to all orders. Phys. Lett. B649, 95-102 (2007)

23. Eichhorn, A., Koslowski, T., Lumma, J., Pereira, A.D.: Towards background independent quantum gravity with tensor models. Class. Quant. Grav. 11 (2018)

24. Eynard, B., Orantin, N.: Invariants of algebraic curves and topological expansion. Commun. Num. Theor. Phys. 1, 347-452 (2007)

25. Eynard, B.: A short overview of the "Topological recursion". arXiv:1412.3286 (2014)

26. Eynard, B.: Counting surfaces volume 70 of progress in mathematical physics. Springer, Berlin (2016)

27. Freidel, L.: Group field theory: an overview. Int. J. Theor. Phys. 44, 1769-1783 (2005)

28. Gurău, R., Ryan, J.P.: Tensor models - a review. SIGMA 8, 020 (2012)

29. Gurău, R.: Colored group field theory. Commun. Math. Phys. 304, 69-93 (2011)

30. Gurău, R.: The Schwinger Dyson equations and the algebra of constraints of random tensor models at all orders. Nucl. Phys. B865, 133-147 (2012)

31. Gurău, R.: A generalization of the Virasoro algebra to arbitrary dimensions. Nucl. Phys. B852, 592614 (2011)

32. Grosse, H., Wulkenhaar, R.: Self-Dual Noncommutative $\varphi^{4}$-theory in four dimensions is a nonperturbatively solvable and non-trivial quantum field theory. Commun. Math. Phys. 329, 1069-1130 (2014) 
33. Hawking, S.W. General relativity: an einstein centenary survey. 746-789 1 (1980)

34. Itoyama, H., Mironov, A., Morozov, A.: Ward identities and combinatorics of rainbow tensor models. JHEP 06, 115 (2017)

35. Krajewski, T., Toriumi, R.: Exact renormalisation group equations and loop equations for tensor models. SIGMA 12, 068 (2016)

36. Krajewski, T.: Group field theories. PoS. QGQGS2011:005 (2011)

37. Krajewski, T.: Schwinger-Dyson Equations in group field theories of quantum gravity. In: 29th international colloquium on group-theoretical methods in physics, p. 11 (2012)

38. Lang, S.: Algebra. Springer, Berlin (2002). Graduate Texts in Mathematics

39. Lins, S., Mulazzani, M.: Blobs and flips on gems. J. Knot Theory Ramif. 15, 1001-1035 (2006)

40. Lahoche, V., Samary, D.O.: Progress in the solving nonperturbative renormalization group for tensorial group field theory. Universe 5, 86 (2019)

41. Lahoche, V., Samary, D.O.: Ward-constrained melonic renormalization group flow for the rank-four $\varphi^{6}$ tensorial group field theory. Phys. Rev. D 100(8), 086009 (2019)

42. Marcolli, M.: Noncommutative cosmology WSP (2018)

43. Mielczarek, J.: Towards the map of quantum gravity. Gen. Rel. Grav. 50(6), 68 (2018)

44. Migdal, A.A.: Loop equations 1/N expansion. Phys. Rep. 102, 199-290 (1983)

45. Oeckl, R.: A local and operational framework for the foundations of physics. arXiv:1610.09052 (2016)

46. Oriti, D.: Group field theory and loop quantum gravity. arXiv:1408.7112 (2014)

47. Samary, D.O., Pérez-Sánchez, C.I., Vignes-Tourneret, F., Wulkenhaar, R.: Correlation functions of a just renormalizable tensorial group field theory: the melonic approximation. Class. Quant. Grav. 32(17), 175012 (2015)

48. Samary, D.O.: Closed equations of the two-point functions for tensorial group field theory. Class. Quant. Grav. 31, 185005 (2014)

49. Pascalie, R.: A solvable tensor field theory. Lett. Math. Phys. 110(0), 925-943 (2020). https://doi.org/10.1007/s11005-019-01245-0

50. Pascalie, R., Pérez-Sánchez, C.I., Tanasa, A., Wulkenhaar, R.: On the large $N$ limit of SchwingerDyson equations of a rank-3 tensor field theory. J. Math. Phys. 60(7), 073502 (2019)

51. Pascalie, R., Pérez-Sánchez, C.I., Wulkenhaar, R.: Correlation functions of U(N)-tensor models and their Schwinger-Dyson equations. arXiv:1706.07358 (2017)

52. Pérez-Sánchez, C.I.: Surgery in colored tensor models. J. Geom. Phys. 120, 262-289 (2017)

53. Pérez-Sánchez, C.I.: The full Ward-Takahashi Identity for colored tensor models. Commun. Math. Phys. 358(2), 589-632 (2018)

54. Pérez-Sánchez, C.I.: Correction to: The full ward-takahashi identity for colored tensor models. Commun. Math Phys. 376, 747-751 (2020). https://doi.org/10.1007/s00220-019-03655-6

55. Pezzana, M.: Sulla struttura topologica delle varietà compatte. Ati Sem. Mat. Fis. Univ. Modena 23(1), 269-277 (1975)

56. Vincent R.: Random tensors and quantum gravity. SIGMA 12, 069 (2016)

57. Rivasseau, V.: The tensor track, III. Fortsch. Phys. 62, 81-107 (2014)

58. Rivasseau, V., Vignes-Tourneret, F.: Constructive tensor field theory: the $t_{4}^{4}$ model. Commun. Math. Phys. 366(2), 567-646 (2019)

59. Rovelli, C.: The strange equation of quantum gravity. Class. Quant. Grav. 32(12), 124005 (2015)

60. Sułkowski, P.: Topological recursion. Snapshots of modern mathematics from Oberwolfach (2) (2018)

61. Ousmane-Samary, D.: Fabien Vignes-Tourneret. Just Renormalizable TGFT's on $U(1)^{d}$ with Gauge Invariance. Commun. Math. Phys. 329, 545-578 (2014)

62. Tanasa, A.: The Multi-Orientable random tensor model, a review. SIGMA 12, 056 (2016)

63. Tutte, W.T.: A census of planar triangulations. Can. J. Math. 14, 21-38 (1962)

64. Tutte, W.T.: A census of planar maps. Can. J. Math. 15, 249-271 (1963)

Publisher's Note Springer Nature remains neutral with regard to jurisdictional claims in published maps and institutional affiliations. 


\section{Affiliations}

\section{Carlos I. Pérez-Sánchez ${ }^{1,2}$}

1 Mathematisches Institut der Westfälischen Wilhelms-Universität, Einsteinstraße 62, 48149 Münster, Germany

2 Faculty of Physics, University of Warsaw, ul. Pasteura 5, 02-093, Warsaw, Poland 